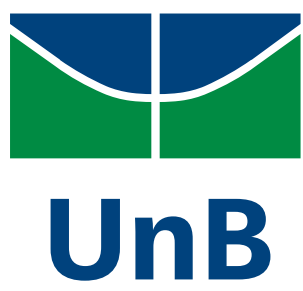

Universidade de Brasília

Instituto de Ciências Exatas

Departamento de Matemática

\title{
Sobre o Fluxo de Curvatura Média em Formas Espaciais
}

por

Hiuri Fellipe Santos dos Reis

Orientadora: Keti Tenenblat

Brasília

2017 
Hiuri Fellipe Santos dos Reis

Sobre o Fluxo de Curvatura Média em Formas Espaciais

Tese apresentada ao Departamento de Matemática da Universidade de Brasília, como parte dos requisitos para a obtenção do grau de DOUTOR EM MATEMÁTICA.

Área de Concentração: Geometria Diferencial

Orientadora: Prof ${ }^{\mathrm{a}}$. Dr ${ }^{\mathrm{a}}$. Keti Tenenblat

Brasilia 
Ficha catalográfica elaborada automaticamente, com os dados fornecidos pelo(a) autor(a)

S S

Santos dos Reis, HIuri Fellipe

Sobre o Fluxo de Curvatura Média em Formas

Espaciais / HIuri Fellipe Santos dos Reis;

orientador Keti Tenenblat. -- Brasília, 2017.

$80 \mathrm{p}$.

Tese (Doutorado - Doutorado em Matemática) -Universidade de Brasília, 2017.

1. Geometria. 2. Geometria Diferêncial. 3. Fluxo de Curvatura Média. I. Tenenblat, Keti, orient. II. Título. 


\section{Sobre o Fluxo de Curvatura Média em Formas Espaciais por}

\section{Hiuri Fellipe Santos dos Reis}

Tese apresentada ao Corpo Docente do Programa de Pós-Graduação em Matemática-UnB, como requisito parcial para obtenção do grau de

\section{DOUTOR EM MATEMÁTICA}

Brasília, 17 de março de 2017.

Comissão Examinadora:

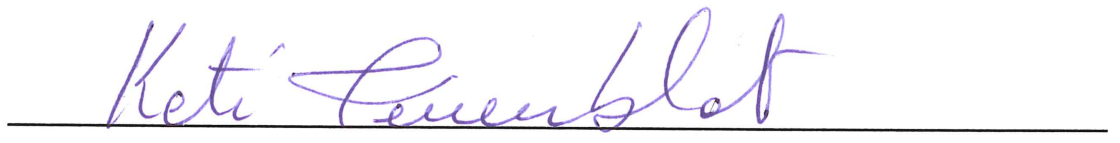

Profa. Dra. Keti Tenenblat - Orientadora (MAT-UnB)

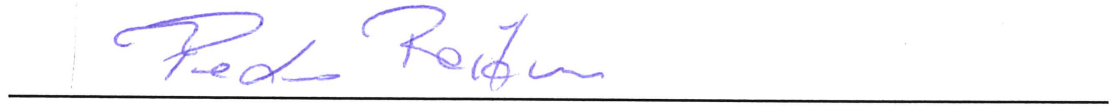

Prof. Dr. Pedro Roitman (MAT-UnB)

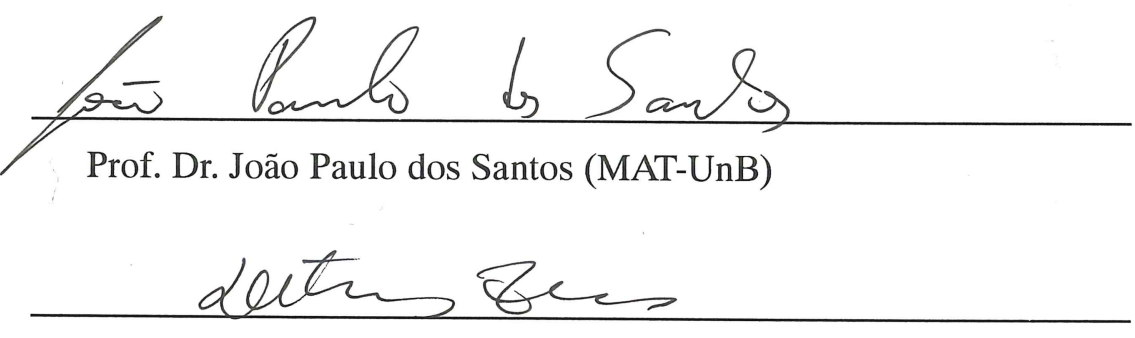

Prof. Dr. Detang Zhou - (UFF/RJ)

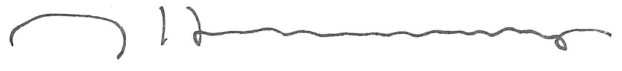

Prof. Dr. Jorge Herbert Soares de Lira - UFC/CE

*O autor foi bolsista CAPES e CNPq durante a elaboração deste trabalho. 
Aos meus pais, Jordelei Antônio Machado e Eleide Maria Lemes. 
Agradecimentos

Agradeço a Deus, em primeiro lugar.

Aos meus pais Jordelei, Eleide, Anderson e Yeda por estarem presentes sempre que precisei. Aos meus avós Antônio (em memória) e Nardair, pelo amor e carinho. Aos meus irmãos, cunhadas e sobrinhos: Jordelei Filho, Hebert, Marcos, Paulo Henrique, Kaio Lucas, João Pedro, Ana Luisa, Ana Julia, Andressa, Priscilla, Sthefany, Lucas e Davi, por fazerem parte da minha vida. Bem como à todos da minha família.

A minha orientadora Keti Tenemblat pela paciência, incentivo e dedicação. Aos professores membros da banca, Detang Zhou, Jorge Lira, Pedro Roitman e João Paulo por tornarem o meu trabalho melhor através de suas críticas e sugestões.

Aos professores e funcionários do Departamento de Matemática da UnB, que foram fundamentais na minha formação. Em especial aos professores Marcelo, Luciana e Tarcísio pelo apoio durante todo o doutorado.

Finalmente, mas não menos importantes, a todos meus colegas de doutorado e amigos. Em especial aos geômetras: Alan, Bruno, Valter, Fábio, Cid, Erikson, Lidiane, Lucas, Hudson, Benedito, e Adriano, pela ajuda e pelo companheirismo. E aos amigos não geômetras: David, Rayner, Daiane, Ludmila, Juliana, Lais, Camila, Alex, Bruno de Paula, Yerko, Dióscoros e Grassielle, pela amizade e por sempre estarem do meu lado. 
"Se eu vi mais longe, foi por estar de pé sobre os ombros de gigantes."

Isaac Newton 
Obtemos condições necessárias e suficientes para que uma curva de $\mathbb{S}^{2}$ seja um sóliton do fluxo redutor de curvas. A partir desse resultado, descrevemos a forma geométrica dos sólitons da esfera bidimensional. Além disso, visualizamos alguns exemplos destas curvas.

Provamos que uma hipersuperfície de uma forma espacial é condição inicial de uma solução do fluxo de curvatura média por hipersuperfícies paralelas se, e somente se, é uma hipersuperfície isoparamétrica. Aplicamos este teorema para obter soluções do fluxo de curvatura média partindo de hipersuperfícies isoparamétricas de formas espaciais.

Palavras-chave: fluxo de curvatura média; fluxo redutor de curvas; forma espacial; hipersuperfície isoparamétrica; hipersuperfície paralela; sóliton. 


Abstract

We obtain necessary and sufficient conditions for a curve in $\mathbb{S}^{2}$ to be a shortening curve flow soliton. From this result, we describe the geometry of the solitons in a twodimensional sphere. In addition, we visualize some examples of such curves.

We prove that, a hypersurface in a space form is an initial condition for a solution of the mean curvature flow by parallel hypersurfaces if, and only if, it is isoparametric. We apply this theorem to obtain solutions of the mean curvature flow starting from isoparametric hypersurfaces of space forms.

Keywords: curve shortening flow; isoparametric hypersurface; mean curvature flow; parallel hypersurface; space form; soliton. 
1 Preliminares 4

1.1 Curvas sobre a Esfera $\mathbb{S}^{2} \ldots \ldots \ldots \ldots \ldots$

1.2 Hipersuperfícies em Formas Espaciais . . . . . . . . . . . . . . . . . 8

1.3 Hipersuperfícies Paralelas em Formas Espaciais . . . . . . . . . . . . . 12

1.4 Fluxo de Curvatura Média . . . . . . . . . . . . . . . . . . . . . . 18

2 Sólitons do Fluxo Redutor de Curvas na Esfera 25

2.1 Caracterização dos Sólitons do FRC na Esfera . . . . . . . . . . . . . . . 26

2.2 Descrição dos Sólitons do FRC na Esfera . . . . . . . . . . . . . . . . . . 29

2.3 Excmplos de Sólitons do FRC na Esfera . . . . . . . . . . . . . . . . . . . 42

3 Soluções do FCM em Formas Espaciais por Hipersuperfícies Paralelas 46

3.1 Teorema de Caracterização . . . . . . . . . . . . . . . . . . . . . . 47

3.2 Soluções do FCM por Hipersuperfícies Paralelas em $\mathbb{R}^{n+1}$. . . . . . . . . 49

3.3 Soluções do FCM por Hipersuperfícies Paralelas em $\mathbb{H}^{n+1}$. . . . . . . . . . 54

3.4 Soluções do FCM por Hipersuperfícies Paralelas em $\mathbb{S}^{n+1} \ldots$. . . . . . . 63

$\begin{array}{ll}\text { Referências } & 66\end{array}$ 


\section{Lista de Figuras}

2.1 Esfera . . . . . . . . . . . . . . . . . . . . 31

2.2 Retrato de Fase de $\psi \ldots \ldots$. . . . . . . . . . . . . . . . . . . . . . . . 39

2.3 Sólitons do FRC na Esfera, $a=0,5 \ldots \ldots . \ldots \ldots$

2.4 Sólitons do FRC na Esfera, $a=1 \ldots \ldots$. . . . . . . . . . . 44

2.5 Sólitons do FRC na Esfera, $a=2 \ldots \ldots . \ldots . \ldots . \ldots . \ldots 4$

2.6 Sólitons do FRC na Esfera, $\mathrm{a}=3 \ldots \ldots$. . . . . . . . . . . . . 45

3.1 Fluxo de Curvatura Média sobre a Esfera Euclidiana . . . . . . . . . . . 51

3.2 Fluxo de Curvatura Média sobre a Cilindro Euclidiano . . . . . . . . . . 53

3.3 Fluxo de Curvatura Média sobre a Horoesfera . . . . . . . . . . . . . . 55

3.4 Fluxo de Curvatura Média sobre a Paralela $(0<|\kappa|<1) \ldots . . . . . .58$

3.5 Fluxo de Curvatura Média sobre a Esfera de Geodésica de $\mathbb{H}^{3}(|\kappa|>1)$. . 59

3.6 Fluxo de Curvatura Média sobre o Cilindro Hiperbólico . . . . . . . . . . 62

3.7 Fluxo de Curvatura Média sobre o Toro de Hopf . . . . . . . . . . . . . . . 67 


\section{Introdução}

O fluxo de curvatura média (FCM) é uma equação diferencial parcial de evolução não linear para hipersuperfícies de uma variedade Riemanniana que se comporta como a equação do calor em um curto período de tempo. No entanto, após um tempo, a parte não linear da equação domina a solução e se torna singular. Além disso, o FCM é um fluxo do tipo gradiente para o funcional área [28]. O estudo das soluções autossimilares são de fundamental importância para a compreensão do fluxo.

O fluxo de curvatura média começou a ser estudado em ciência de materiais na década de 1950. Mullis [29], em seu estudo sobre metais, viu que as interfaces do metal geralmente não tem curvatura média constante. Como consequência, Mullis provavelmente foi o primeiro a escrever a equação do fluxo. O FCM e outros fluxos relacionados vem sendo estudados em varias áreas do conhecimento, como análise geométrica [12, 22], relatividade geral $[11,19]$, e topologia $[2,10]$.

Hipersuperfícies que evoluem de forma autossimilar sobre o FCM, isto é, por uma composição de isometrias e homotetias, desempenham um papel importante na teoria de singularidades do fluxo. As mais importantes são as hipersuperfícies autocontráteis (selfshrinking) e as hipersuperfícies que fluem por traslação, elas são modelos de singularidades do fluxo [20, 23].

Quando aplicado às curvas de uma variedade Riemanniana bidimensional, o FCM é 
conhecido como fluxo redutor de curvas (FRC). As soluções autossimilares para o FRC no plano são bem conhecidas. As duas soluções mais simples são as retas, que não são afetadas pelo fluxo, e os círculos que encolhem homoteticamente a um ponto em tempo finito. Uma outra solução simples é a curva Grim Reaper, gráfico da função $f(x)=\ln \cos (x)$ (veja Exemplo 1.26), que é a única curva de translação do plano [15]. Em [4], Altschuler mostrou uma curva que gira a uma velocidade constante denominada espiral yin-yang. Além do círculos, existem outras curvas que encolhem homoteticamente ao longo do fluxo, elas foram classificadas por Abresch e Langer em [1]. Uma discussão sobre as curvas que expandem desta forma pode ser encontrado em [24, 35]. Finalmente, em [16] Halldorsson deu uma completa classificação das soluções autossimilares do FRC no plano Euclidiano.

Ainda no espaço Euclidiano, para dimensões maiores, os exemplos mais simples de soluções autossimilares são as esferas e os cilindros, elas são hipersuperfícies autocontráteis (veja os Exemplos 1.20 e 1.21). Outros exemplos de hipersuperfícies autocontráteis no espaço Euclidiano podem ser encontradas em [3, 7, 25] e hipersuperfícies de translação são encontradas em $[8,30,36,17]$.

Há poucos estudos sobre soluções do fluxo de curvatura média em espaços não Euclidianos. Em [21], Hungerbühler e Smoczyk consideraram um caso particular de soluções autossimilares que evoluem pelo FCM por um grupo de isometrias do espaço ambiente, que são conhecidas como sólitons, e apresentaram vários exemplos destas hipersuperfícies sobre variedades Riemannianas. Em [27], Liu e Terng estudaram o FCM sobre subvariedades isoparamétricas do espaço Euclidiano e da esfera, onde provaram que o fluxo preserva a condição de ser isoparamétrica e desenvolve singularidade em tempo finito.

Este trabalho tem dois objetivos principais: o primeiro consiste no estudo dos sólitons do fluxo redutor de curvas em $\mathbb{S}^{2}$, isto é, curvas que evoluem pelo FRC por uma família a um parâmetro de isometrias de $\mathbb{S}^{2}$; o segundo é o estudo das soluções do fluxo de curvatura média por hipersuperfícies paralelas em formas espaciais.

Provamos que uma curva de $\mathbb{S}^{2} \subset \mathbb{R}^{3}$ é um sóliton do FRC se, e somente se, sua curvatura é proporcional ao ângulo entre o vetor tangente e um vetor fixo de $\mathbb{R}^{3} \backslash\{0\}$ (Teorema 2.1). Esta caracterização é dada em termos de sistemas de equações diferenciais ordinárias (Proposições 2.2 e 2.18), que permitem obter a descrição geométrica dos sólitons 
do FRC (Teorema 2.17) e permitem também visualizar tais sólitons.

No estudo do FCM para hipersuperfícies de formas espacias $\mathbb{M}^{n+1}(\bar{\kappa})$, provamos que uma imersão $F: M \rightarrow \mathbb{M}^{n+1}(\bar{\kappa})$ evolui pelo fluxo de curvatura média por hipersuperfícies paralelas se, e somente se, $F(M)$ é isoparamétrica (Teorema 3.2). Aplicações da teoria permitem obter soluções do FCM partindo de hipersuperfícies isoparamétricas de formas espaciais.

No capítulo 1, apresentamos resultados básicos sobre curvas na esfera $\mathbb{S}^{2}$ e hipersuperfícies em formas espaciais, em particular sobre hipersuperfícies paralelas e isoparamétricas. Apresentamos também resultados gerais sobre o fluxo de curvatura média e mostramos uma equivalência local entre duas definições do FCM existentes na literatura.

No Capitulo 2, provamos uma condição necessária e suficiente para que uma curva da esfera seja um sóliton do FRC em $\mathbb{S}^{2}$, este teorema nos fornece sistemas de equações diferenciais ordinárias que caracterizam os sólitons. Em seguida, usamos esta equação para descrever todos os sólitons do FRC da esfera, dando uma descrição destas curvas através de uma sequência de lemas. Para finalizar este capítulo, usamos métodos numéricos e computação gráfica, para visualizar alguns exemplos de sólitons do FRC em $\mathbb{S}^{2}$.

No Capítulo 3, provamos que uma hipersuperfície de uma forma espacial admite uma solução para o fluxo de curvatura média por hipersuperfícies paralelas se, e somente se, é isoparamétrica. Aplicando o teorema, construímos soluções para o fluxo de curvatura média por hipersuperfícies paralelas sobre hipersuperfícies isoparamétricas de formas espaciais. Em particular, no espaço hiperbólico, provamos que as horoesferas não mudam sua forma ao longo do fluxo, que as esferas geodésicas colapsam em um ponto, as hipersuperfícies equidistantes convergem para uma hipersuperfície totalmente geodésica, e que os cilindros hiperbólicos colapsam em uma variedade totalmente geodésica. 


\section{CAPÍTULO 1}

\section{Preliminares}

\subsection{Curvas sobre a Esfera $\mathbb{S}^{2}$}

Nesta seção, vamos apresentar algumas definições e fatos básicos sobre curvas na esfera que serão usados ao longo do Capítulo 2.

Vamos considerar a esfera $\mathbb{S}^{2}$ sendo a superfície do espaço Euclidiano $\mathbb{R}^{3}$, dada por

$$
\mathbb{S}^{2}=\left\{\mathbf{x}=\left(x_{1}, x_{2}, x_{3}\right) \in \mathbb{R}^{3} \mid\langle\mathbf{x}, \mathbf{x}\rangle=x_{1}^{2}+x_{2}^{2}+x_{3}^{2}=1\right\},
$$

com sua a métrica induzida de $\mathbb{R}^{3}$.

Definição 1.1 Uma curva parametrizada regular em $\mathbb{S}^{2}$ é uma imersão diferenciável $X$ : $I \rightarrow \mathbb{S}^{2}$, definida sobre um intervalo dos números reais $I \subset \mathbb{R}$, tal que $X^{\prime}(s) \neq 0$, para todo $s \in \mathbb{R}$.

A menos que mencionemos o contrário, vamos considerar $X$ parametrizada pelo comprimento de arco. O campo tangente unitário é definido por

$$
T(s)=X^{\prime}(s)=\frac{d}{d s} X(s),
$$

e escolhemos o campo normal unitário a $X(s)$ no espaço tangente $T_{X(s)} \mathbb{S}^{2}$

$$
N(s)=T(s) \wedge X(s) .
$$


Definição 1.2 O número real $\kappa(s)$ definido por $\kappa(s)=\left\langle T^{\prime}(s), N(s)\right\rangle$ é denominado a curvatura (geodésica) da curva $X$ no instante $s$.

Como o vetor posição é ortogonal à esfera, o triedro $\{T(s), N(s), X(s)\}$ é um referencial ortonormal ao longo da curva. Este referencial é denominado triedro de Darboux da curva $X$. O triedro de Darboux satisfaz as equações

$$
\left\{\begin{array}{l}
T^{\prime}(s)=\kappa(s) N(s)-X(s) \\
N^{\prime}(s)=-\kappa(s) T(s) \\
X^{\prime}(s)=T(s)
\end{array}\right.
$$

denominadas fórmulas de Darboux.

Este sistema nos permite provar o Teorema Fundamental das Curvas na Esfera que vamos apresentar agora.

Teorema 1.3 (Teorema Fundamental das Curvas na Esfera) Dada uma função $\kappa: I \rightarrow$ $\mathbb{R}$, existe uma curva parametrizada regular $X: I \rightarrow \mathbb{S}^{2}$, tal que s é o parâmetro por comprimento de arco e $\kappa$ a curvatura de $X$. Além disso, qualquer outra curva $\widetilde{X}$ satisfazendo as mesmas condições, difere de $X$ por uma isometria de $\mathbb{S}^{2}$, isto é, existe uma transformação ortogonal $A \in o(3)$, tal que $\widetilde{X}(s)=A X(s)$.

Prova. Observe que o triedro de Darboux pode ser visto como um sistema de equações diferenciais ordinárias em $I \times \mathbb{R}^{9}$

$$
\left\{\begin{array}{l}
t_{\imath}^{\prime}(s)=\kappa(s) n_{\imath}(s)-x_{\imath}(s) \\
n_{\imath}^{\prime}(s)=-\kappa(s) t_{\imath}(s) \\
x_{\imath}^{\prime}(s)=t_{\imath}(s)
\end{array}\right.
$$

com $\imath=1,2,3$, onde $T=\left(t_{1}, t_{2}, t_{3}\right), N=\left(n_{1}, n_{2}, n_{3}\right)$, e $X=\left(x_{1}, x_{2}, x_{3}\right)$.

Assim, pelo teorema de existência e unicidade de soluções para equações diferenciais ordinárias, dado um triedro ortonormal $\left\{T_{0}, N_{0}, X_{0}\right\}$ e $s_{0} \in I$, existe uma família de triedros

$$
\{T(s), N(s), X(s)\}, s \in I,
$$

satisfazendo $T\left(s_{0}\right)=T_{0}, N\left(s_{0}\right)=N_{0}$, e $X\left(s_{0}\right)=X_{0}$. 
Agora, vamos mostrar que $\{T(s), N(s), X(s)\}$ permanece ortonormal para todo $s \in I$. De fato, utilizando (1.1) para expressar as derivadas das seis quantidades

$$
\langle T, N\rangle,\langle T, X\rangle,\langle N, X\rangle,\langle T, T\rangle,\langle N, N\rangle \text { e }\langle X, X\rangle
$$

como funções destas mesmas quantidades, obtemos o sistema de equações diferenciais ordinárias

$$
\left\{\begin{array}{l}
\langle T, N\rangle^{\prime}=\kappa\langle N, N\rangle-\langle X, N\rangle-\kappa\langle T, T\rangle, \\
\langle T, X\rangle^{\prime}=\kappa\langle N, X\rangle-\langle X, X\rangle+\langle T, T\rangle, \\
\langle N, X\rangle^{\prime}=-\kappa\langle T, X\rangle+\langle T, N\rangle, \\
\langle T, T\rangle^{\prime}=2 \kappa\langle T, N\rangle-2\langle T, N\rangle, \\
\langle N, N\rangle^{\prime}=-2 \kappa\langle T, N\rangle, \\
\langle X, X\rangle^{\prime}=2\langle T, X\rangle .
\end{array}\right.
$$

Por verificação direta, podemos ver que

$$
\langle T, N\rangle \equiv 0,\langle T, X\rangle \equiv 0,\langle N, X\rangle \equiv 0,\langle T, T\rangle \equiv 1,\langle N, N\rangle \equiv 1,\langle X, X\rangle \equiv 1
$$

é solução para o sistema acima com condições iniciais $0,0,0,1,1,1$, respectivamente. por unicidade, a família $\{T(s), N(s), X(s)\}$ é ortonormal para todo $s \in I$.

Por (1.1), temos que $X^{\prime}=T$ e $T^{\prime}=\kappa N-X$, isso implica que $\kappa$ é a curvatura da curva $X$.

Agora precisamos mostrar que $X$ é única a menos de isometria. Seja $\widetilde{X}: I \rightarrow \mathbb{S}^{2}$ outra curva com curvatura $\widetilde{\kappa}(s)=\kappa(s)$, para todo $s \in I$, e seja $\left\{\widetilde{T}_{0}, \widetilde{N}_{0}, \widetilde{X}_{0}\right\}$ o triedro de Darboux de $\tilde{X}$ em $s_{0}$. Existe um aplicação ortogonal $A \in O(3)$ que leva a base $\left\{T_{0}, N_{0}, X_{0}\right\}$ em $\left\{\widetilde{T}_{0}, \widetilde{N}_{0}, \widetilde{X}_{0}\right\}$. A curva $A X$, tem curvatura $\kappa$ e em $s_{0}$ ela satisfaz as condições iniciais $\left\{\widetilde{T}_{0}, \widetilde{N}_{0}, \widetilde{X}_{0}\right\}$. Portando, pela unicidade provada anteriormente, temos que $\widetilde{X}=A X$.

Seja $u \in \mathbb{R}^{3}$ um vetor não nulo do espaço Euclidiano. Ao longo deste trabalho, será conveniente trabalhar com as seguintes funções ao longo da curva $X$ na esfera

$$
\tau(s)=\langle T(s), u\rangle, \nu(s)=\langle N(s), u\rangle \text { e } \alpha(s)=\langle X(s), u\rangle .
$$


Por uma aplicação direta das formulas de Darboux, podemos verificar que estas funções satisfazem as seguintes equações.

$$
\left\{\begin{aligned}
\tau^{\prime}(s) & =\kappa(s) \nu(s)-\alpha(s) \\
\nu^{\prime}(s) & =-\kappa(s) \tau(s) \\
\alpha^{\prime}(s) & =\tau(s)
\end{aligned}\right.
$$

No Capitulo 2, vamos encontrar todas as curvas $X$ satisfazendo uma equação da forma $\kappa(s)=\Phi(\tau(s), \nu(s))$ usando a seguinte proposição.

Proposição 1.4 Sejam $\Phi: \mathbb{R}^{2} \rightarrow \mathbb{R}$ uma função suave, $\tau_{0}, \nu_{0}$, e $\alpha_{0}$ números reais, e $u \in \mathbb{R}^{3}$ um vetor não nulo, tais que

$$
\tau_{0}^{2}+\nu_{0}^{2}+\alpha_{0}^{2}=\|u\|^{2}
$$

Então, a menos de isometria de $\mathbb{S}^{2}$, existe uma única curva $X: \mathbb{R} \rightarrow \mathbb{S}^{2}$, definida para toda reta, tal que

$$
\kappa(s)=\Phi(\tau(s), \nu(s))
$$

onde

$$
\tau(s)=\langle T(s), u\rangle, \quad \nu(s)=\langle N(s), u\rangle \quad e \quad \alpha(s)=\langle X(s), u\rangle,
$$

para todo $s \in \mathbb{R}$ e $\tau(0)=\tau_{0}, \nu(0)=\nu_{0}$ e $\alpha(0)=\alpha_{0}$.

Prova. Tendo em vista (1.3), sejam $\tau, \nu$, e $\alpha$ as únicas soluções para o sistema de equações diferenciais ordinárias

$$
\left\{\begin{array}{l}
\tau^{\prime}=\Phi(\tau, \nu) \nu-\alpha \\
\nu^{\prime}=-\Phi(\tau, \nu) \tau \\
\alpha^{\prime}=\tau
\end{array}\right.
$$

com condições iniciais $\tau(0)=\tau_{0}, \nu(0)=\nu_{0}$ e $\alpha(0)=\alpha_{0}$. Como (1.4) é satisfeito e

$$
\begin{aligned}
\frac{d}{d s}\left(\tau^{2}+\nu^{2}+\alpha^{2}\right) & =2 \tau \tau^{\prime}+2 \nu \nu^{\prime}+2 \alpha \alpha^{\prime} \\
& =2 \tau(\Phi \nu-\alpha)-2 \nu \Phi \tau+2 \alpha \tau \\
& =0
\end{aligned}
$$


temos que $\tau^{2}(s)+\nu^{2}(s)+\alpha^{2}(s)=\|u\|^{2}$. Assim, como a solução não tende para infinito em tempo finito, ela esta definida para todo $s \in \mathbb{R}$.

Defina $\kappa(s)=\Phi(\tau(s), \nu(s))$, pelo Teorema 1.3, existe uma única curva $X: \mathbb{R} \rightarrow \mathbb{S}^{2}$, a menos de posição na esfera, tal que a curvatura de $X$ é dada por $\kappa$ e as condições iniciais satisfazem

$$
\tau(0) T(0)+\nu(0) N(0)+\alpha(0) X(0)=u \text {. }
$$

Além disso

$$
\begin{aligned}
\frac{d}{d s}(\tau T+\nu N+\alpha X) & =\tau^{\prime} T+\tau T^{\prime}+\nu^{\prime} N+\nu N^{\prime}+\alpha^{\prime} X+\alpha X^{\prime} \\
& =(\kappa \nu-\alpha) T+\tau(\kappa N-X)-\kappa \tau N-\kappa \nu T+\tau X+\alpha T \\
& =0,
\end{aligned}
$$

concluímos que

$$
\tau(s) T(s)+\nu(s) N(s)+\alpha(s) X(s)=u,
$$

e assim temos o resultado.

\subsection{Hipersuperfícies em Formas Espaciais}

Nesta seção, vamos apresentar algumas definições e fatos básicos sobre hipersuperfícies em formas espaciais. Além disso, vamos apresentar alguns teoremas de classificação das hipersuperfícies isoparamétricas das formas espaciais.

Vamos considerar as formas espaciais

$$
\mathbb{M}^{n+1}(\bar{\kappa})= \begin{cases}\mathbb{R}^{n+1} & \text { se } \bar{\kappa}=0, \\ \mathbb{S}^{n+1} \subset \mathbb{R}^{n+2} & \text { se } \bar{\kappa}=1, \\ \mathbb{H}^{n+1} \subset \mathbb{L}^{n+2} & \text { se } \bar{\kappa}=-1,\end{cases}
$$

onde $\mathbb{L}^{n+2}$ é o espaço de Lorentz, isto é,

$$
\mathbb{L}^{n+2}=\left\{\mathbf{x}=\left(x_{1}, x_{2}, \ldots, x_{n+2}\right) \mid \mathbf{x} \in \mathbb{R}^{n+2}\right\}
$$


munido do produto interno

$$
\langle\mathbf{x}, \mathbf{y}\rangle=-x_{1} y_{1}+\sum_{\jmath=2}^{n+2} x_{\jmath} y_{\jmath}
$$

O espaço hiperbólico é a subvariedade

$$
\mathbb{H}^{n+1}=\left\{\mathbf{x} \in \mathbb{L}^{n+2} \mid\langle\mathbf{x}, \mathbf{x}\rangle=-1 \text { e } x_{1} \geq 1\right\}
$$

e a esfera é a subvariedade

$$
\mathbb{S}^{n+1}=\left\{\mathbf{x} \in \mathbb{R}^{n+2} \mid\langle\mathbf{x}, \mathbf{x}\rangle=1\right\}
$$

com a métrica induzida do espaço ambiente.

Definição 1.5 Uma hipersuperfície na forma espacial $\mathbb{M}^{n+1}(\bar{\kappa})$ é uma imersão $F: M^{n} \rightarrow$ $\mathbb{M}^{n+1}(\bar{\kappa})$, com a métrica induzida

$$
g_{x}(v, w)=\left\langle d F_{x}(v), d F_{x}(w)\right\rangle_{F(x)},
$$

para todo $x \in M$ e $v, w \in T_{x} M$. A forma quadrática definida por $g$ é denominada primeira forma fundamental da hipersuperfície $F(M)$, em $x \in M^{n}$.

Para simplificar a notação identificamos $M \operatorname{com} F(M)$, e cada vetor $v \in T_{x} M, x \in M$, $\operatorname{com} d F_{x}(v) \in T_{F(x)} F(M)$.

Uma hipersuperfície $F: M^{n} \rightarrow \mathbb{M}^{n+1}(\bar{\kappa})$ é orientável, se existe um campo normal unitário $N: M \rightarrow(T M)^{\perp}$, definido sobre toda a hipersuperfície, onde $(T M)^{\perp}$ denota o espaço normal a $F(M)$ em $T \mathbb{M}$. Neste caso dizemos que $N(x)$ é a aplicação normal de Gauss de $F(M)$ em $x$.

Definição 1.6 Seja $F: M^{n} \rightarrow \mathbb{M}^{n+1}(\bar{\kappa})$ uma hipersuperfície orientada. A forma quadrática $h_{x}$ sobre $T_{x} M$ definida por

$$
h_{x}(v, w)=-\left\langle d N_{x}(v), d F_{x}(w)\right\rangle_{F(x)},
$$

é denominada segunda forma fundamental da hipersuperfície $F(M)$, em $x \in M^{n}$. 
Seja $x \in M$, como a segunda forma fundamental $h$ é simétrica, existe uma base ortonormal de autovetores $\left\{e_{1}, \ldots, e_{n}\right\}$ de $T_{x} M$ com autovalores $\kappa_{1}, \ldots, \kappa_{n}$, isto é,

$$
g_{x}\left(e_{\imath}, e_{\jmath}\right)=\delta_{\imath \jmath} \text { e } h_{x}\left(e_{\imath}, e_{\jmath}\right)=\kappa_{\imath}(x) \delta_{\imath \jmath}
$$

para todo $1 \leq \imath, \jmath \leq n$. Neste caso, denominamos os vetores $e_{\imath}$ direções principais e os $\kappa_{\imath}(x)$ curvaturas principais de $F(M)$ em $x$.

Para definir as $r$-curvaturas médias, vamos fazer uso das funções simétricas elementares que introduziremos agora.

Seja $\sigma_{r}\left(\mu_{1}, \ldots, \mu_{l}\right) \in \mathbb{R}\left[\mu_{1}, \ldots, \mu_{l}\right]$ o $r$-ésimo polinômio simétrico elementar sobre $\mu_{1}, \ldots, \mu_{l}$, isto é,

- $\sigma_{0}\left(\mu_{1}, \ldots, \mu_{l}\right)=1$,

- $\sigma_{r}\left(\mu_{1}, \ldots \mu_{l}\right)=\sum_{1 \leq \imath_{1}<\ldots<\imath_{r} \leq l} \mu_{\imath_{1}} \mu_{\imath_{2} \ldots} \ldots \mu_{\imath_{r}}$ para $1 \leq r \leq l$,

- $\sigma_{r}\left(\mu_{1}, \ldots, \mu_{l}\right)=0$, para $r>l$.

A respeito dos polinômios simétricos elementares, temos o seguinte resultado que será utilizado para demonstrar o Lema 1.16.

Proposição 1.7 Sejam $\sigma_{r}\left(\mu_{1}, \ldots, \mu_{l}\right) \in \mathbb{R}\left[\mu_{1}, \ldots, \mu_{l}\right]$ e $\sigma_{r}\left(\mu_{1}, \ldots, \mu_{l}, \mu_{l+1}\right) \in \mathbb{R}\left[\mu_{1}, \ldots, \mu_{l}, \mu_{l+1}\right]$, para todo $l, r \geq 1$, então

$$
\sigma_{r}\left(\mu_{1}, \ldots, \mu_{l}, \mu_{l+1}\right)=\sigma_{r}\left(\mu_{1}, \ldots, \mu_{l}\right)+\mu_{l+1} \sigma_{r-1}\left(\mu_{1}, \ldots, \mu_{l}\right)
$$

Prova. Para $r=1$, temos

$$
\sigma_{1}\left(\mu_{1}, \ldots, \mu_{l+1}\right)=\mu_{1}+\ldots+\mu_{l}+\mu_{l+1}=\sigma_{1}\left(\mu_{1}, \ldots, \mu_{l}\right)+\mu_{l+1} \sigma_{0}\left(\mu_{1}, \ldots, \mu_{l}\right) .
$$

Agora, para $r>1$, temos

$$
\begin{aligned}
\sigma_{r}\left(\mu_{1}, \ldots, \mu_{l}, \mu_{l+1}\right) & =\sum_{1 \leq \imath_{1}<\ldots<\imath_{r} \leq l+1} \mu_{\imath_{1}} \mu_{\imath_{2}} \ldots \mu_{\imath_{r}} \\
& =\sum_{\substack{1 \leq \imath_{1}<\ldots<\imath_{r} \leq l \\
u_{1}}} \mu_{\imath_{2}} \ldots \mu_{\imath_{r}}+\mu_{l+1} \sum_{\substack{1 \leq \imath_{1}<\ldots<l_{r-1} \leq l\\
}} \mu_{\imath_{1}} \mu_{\imath_{2}} \ldots \mu_{\imath_{r-1}} \\
& \sigma_{r}\left(\mu_{1}, \ldots, \mu_{l}\right)+\mu_{l+1} \sigma_{r-1}\left(\mu_{1}, \ldots, \mu_{l}\right) .
\end{aligned}
$$


Definição 1.8 Seja $F: M^{n} \rightarrow \mathbb{M}^{n+1}$ uma hipersuperfície orientada. A r-curvatura média $S_{r}$ da hipersuperfície $F(M)$ em $x$ é definida por

$$
S_{r}(x)=\sigma_{r}\left(\kappa_{1}(x), \ldots, \kappa_{n}(x)\right)
$$

onde $\kappa_{\imath}(x)$ é a $\imath$-ésima curvatura principal de $F(M)$ em $x$, para $\imath=1, \ldots, n$.

Quando todas as curvaturas principais $\kappa_{\imath}$ de $F(M)$ são independentes de $x$, para todo $\imath=1, \ldots, n$, dizemos que $F(M)$ é uma hipersuperfície isoparamétrica. Isto é equivalente a ter todas as $r$-curvaturas médias $S_{r}(x)$ de $F(M)$ constantes.

As hipersuperfícies isoparamétricas foram muitos estudadas ao longo dos tempos. Temos os seguintes resultado de classificação para estas hipersuperfícies.

Teorema $1.9([33,31])$ Seja $F: M^{n} \rightarrow \mathbb{R}^{n+1}$ uma hipersuperfície isoparamétrica em $\mathbb{R}^{n+1}$, então $F(M)$ é isométrica a uma das seguintes hipersuperfícies:

1. $\mathbb{R}^{n}$, com todas curvaturas principais iguais a $\kappa=0$,

2. $\mathbb{S}^{n}$, con todas as curvaturas iguais a $\kappa \neq 0$,

3. $\mathbb{S}^{m} \times \mathbb{R}^{n-m}$, com duas curvaturas distintas $\kappa_{1} \neq 0$ e $\kappa_{2}=0$.

Este resultado foi primeiramente demostrado para $n=2$ por Somiglina [33] em 1919 e para $n$ arbitrário por B. Segre [31] em 1938. O resultado similar, devido a Cartan [6] (1938) também é valido em $\mathbb{H}^{n+1}$.

Teorema 1.10 ([6]) Seja $F: M^{n} \rightarrow \mathbb{H}^{n+1}$ uma hipersuperfície isoparamétrica do espaço hiperbólico $\mathbb{H}^{n+1}$, então $F(M)$ é isométrico a uma das seguintes hipersuperfícies:

1. $\mathbb{H}^{n}$, com todas curvaturas iguais a $\kappa=0$,

2. $\mathbb{H}^{n}$, com todas as curvaturas principais iguais a $0<|\kappa|<1$,

3. $\mathbb{R}^{n}$, com todas as curvaturas principais iguais a $\kappa= \pm 1$,

4. $\mathbb{S}^{n}$, com todas as curvaturas principais iguais a $|\kappa|>1$, 
5. $\mathbb{S}^{m} \times \mathbb{H}^{n-m}$, com duas curvaturas principais distintas $\kappa_{1}$ e $\kappa_{2}$, satisfazendo $\kappa_{1} \kappa_{2}=1$.

Na classificação acima, quando todas as curvaturas são iguais a $\kappa$, se $\kappa=0$ a hipersuperfície é totalmente geodésica, se $0<|\kappa|<1$ a hipersuperfície é paralela a uma hipersuperfície totalmente geodésica, se $\kappa= \pm 1$ a hipersuperfície é a horoesfera e se $|\kappa|>1$ temos a esfera totalmente geodésica.

A teoria de classificação de hipersuperfícies isoparamétricas na esfera $\mathbb{S}^{n+1}$ é mais complicada, sendo ainda um problema em aberto. Vários trabalhos já foram publicados sobre o assunto, para $n=2$ Cartan [6] em 1938 deu a seguinte classificação.

Teorema 1.11 ([6]) Seja $F: M^{2} \rightarrow \mathbb{S}^{3}$ uma superfície isoparamétrica de $\mathbb{S}^{3}$, então $F(M)$ é isométrico a uma das seguintes superfícies:

1. $\mathbb{S}^{2}$, com curvaturas principais iguais am $\kappa=0$,

2. $\mathbb{S}^{2}$, com curvaturas principais $\kappa \neq 0$,

3. $\mathbb{S}^{1} \times \mathbb{S}^{1}$, com curvaturas principais $\kappa_{1}$ e $\kappa_{2}$ satisfazendo $\kappa_{1} \kappa_{2}=-1$.

Nesta classificação, quando todas as curvaturas de $F(M)$ são iguais a $\kappa$, se $\kappa=0$ temos as esferas totalmente geodésicas, e se $\kappa \neq 0$ temos as esferas totalmente umbílicas. Quando existem duas curvaturas principais distintas, temos o toro de Hopf sobre círculos.

\subsection{Hipersuperfícies Paralelas em Formas Espaciais}

Nesta seção, vamos estudar hipersuperfícies paralelas a uma hipersuperfície dada, relacionando suas propriedades geométricas com as propriedades geométricas da hipersuperfície original. Os resultados obtidos nesta seção serão de fundamental importância no desenvolvimento do Capitulo 3.

Definição 1.12 Seja $F: M^{n} \rightarrow \mathbb{M}^{n+1}(\bar{\kappa})$ uma hipersuperfície orientada na forma espacial $\mathbb{M}^{n+1}(\bar{\kappa})$, com campo vetorial normal unitário $N$. A hipersuperfície paralela à $F(M)$ a uma distância, com sinal, $\xi \in \mathbb{R}$ é a aplicação $\widetilde{F}^{\xi}: M^{n} \rightarrow \mathbb{M}^{n+1}(\bar{\kappa})$ tal que para cada ponto $x \in M^{n}$, o ponto $\widetilde{F}^{\xi}(x)$ é obtido por transladar a distância com sinal $\xi \in \mathbb{R}$ ao longo da geodésica em $\mathbb{M}^{n+1}(\bar{\kappa})$ com ponto inicial $F(x)$ e vetor tangente inicial $N(x)$. 
Quando $\bar{\kappa}=0$ e $\mathbb{M}^{n+1}=\mathbb{R}^{n+1}$, a expressão de $\widetilde{F}^{\xi}$ é dada por

$$
\widetilde{F}^{\xi}(x)=F(x)+\xi N(x),
$$

se $\bar{\kappa}=1$ e $\mathbb{M}^{n+1}=\mathbb{S}^{n+1} \subset \mathbb{R}^{n+2}$, a expressão é dada por

$$
\widetilde{F}^{\xi}(x)=\cos (\xi) F(x)+\sin (\xi) N(x),
$$

se $\bar{\kappa}=-1$ e $\mathbb{M}^{n+1}=\mathbb{H}^{n+1} \subset \mathbb{L}^{n+2}$, a expressão é dada por

$$
\widetilde{F}^{\xi}(x)=\cosh (\xi) F(x)+\sinh (\xi) N(x) .
$$

Assim, podemos escrever $\widetilde{F}^{\xi}$ como

$$
\widetilde{F}^{\xi}(x)=c(\xi) F(x)+s(\xi) N(x)
$$

onde introduzimos a notação

$$
c(\xi)=\left\{\begin{array}{ll}
1, & \text { se } \bar{\kappa}=0 \\
\cos (\xi), & \text { se } \bar{\kappa}=1 \\
\cosh (\xi), & \text { se } \bar{\kappa}=-1
\end{array} \quad \text { e } s(\xi)= \begin{cases}\xi, & \text { se } \bar{\kappa}=0 \\
\sin (\xi), & \text { se } \bar{\kappa}=1 \\
\sinh (\xi), & \text { se } \bar{\kappa}=-1\end{cases}\right.
$$

Observação 1.13 As funções $c(\xi)$ e $s(\xi)$ satisfazem:

- $c^{\prime}(\xi)=-\bar{\kappa} s(\xi)$

- $s^{\prime}(\xi)=c(\xi)$,

- $c^{2}(\xi)+\bar{\kappa} s^{2}(\xi)=1$.

A seguir, vamos obter o campo normal unitário da hipersuperfície paralela $\widetilde{F}^{\xi}(M)$ em termos de $F(M)$.

Lema 1.14 Sejam $F: M^{n} \rightarrow \mathbb{M}^{n+1}(\bar{\kappa})$ uma hipersuperfície orientada, com campo vetorial normal unitário $N$ e $\widetilde{F}^{\xi}: M^{n} \rightarrow \mathbb{M}^{n+1}(\bar{\kappa})$ uma hipersuperfície paralela. Então, a menos de sinal, o campo normal unitário de $\widetilde{F}^{\xi}(M)$ é dado por

$$
\widetilde{N}^{\xi}(x)=-\bar{\kappa} s(\xi) F(x)+c(\xi) N(x) .
$$


Prova. Primeiro vamos mostra que $-\bar{\kappa} s(\xi) F(x)+c(\xi) N(x)$ é normal a $\widetilde{F}_{\xi}\left(M^{n}\right)$. Seja $v \in T_{x} M$, temos que

$$
d \widetilde{F}_{x}^{\xi}(v)=c(\xi) d F_{x}(v)+s(\xi) d N_{x}(v)
$$

assim

$$
\begin{aligned}
\left\langle d \widetilde{F}_{x}^{\xi}(v),-\bar{\kappa} s(\xi) F(x)+c(\xi) N(x)\right\rangle= & \left\langle c(\xi) d F_{x}(v)+s(\xi) d N_{x}(v),-\bar{\kappa} s(\xi) F(x)+c(\xi) N(x)\right\rangle \\
= & -\bar{\kappa} s(\xi) c(\xi)\left\langle d F_{x}(v), F(x)\right\rangle+c^{2}(\xi)\left\langle d F_{x}(v), N(x)\right\rangle \\
& -\bar{\kappa} s^{2}(\xi)\left\langle d N_{x}(v), F(x)\right\rangle+s(\xi) c(\xi)\left\langle d N_{x}(v), N(x)\right\rangle .
\end{aligned}
$$

Como $\|N(x)\|=1$ segue que $\left\langle d N_{x}(v), N(x)\right\rangle=0$. Além disso, $\left\langle d F_{x}(v), N(x)\right\rangle=0$ e se $\bar{\kappa} \neq 0, F(x)$ é ortogonal a $T_{F(x)} \mathbb{M}^{n+1}$. Assim, concluímos que

$$
\left\langle d \widetilde{F}_{x}^{\xi}(v),-\bar{\kappa} s(\xi) F(x)+c(\xi) N(x)\right\rangle=0 .
$$

Agora, vamos mostrar que $-\bar{\kappa} s(\xi) F(x)+c(\xi) N(x)$ é unitário.

$$
\begin{aligned}
\|-\bar{\kappa} s(\xi) F(x)+c(\xi) N(x)\|^{2} & =\bar{\kappa}^{2} s^{2}(\xi)\|F(x)\|^{2}-2 \bar{\kappa} s(\xi) c(\xi)\langle F(x), N(x)\rangle+c^{2}(\xi)\|N(x)\|^{2} \\
& =\bar{\kappa} s^{2}(\xi)+c^{2}(\xi)=1
\end{aligned}
$$

No próximo lema, vamos obter a primeira forma quadrática de $\widetilde{F}^{\xi}(M)$ e vamos usar a expressão para o campo normal unitário acima determinada para relacionar as curvaturas das duas variedades.

Lema 1.15 Sejam $F: M^{n} \rightarrow \mathbb{M}^{n+1}(\bar{\kappa})$ uma hipersuperfície orientada, com campo vetorial normal unitário $N$ e $\widetilde{F}^{\xi}: M^{n} \rightarrow \mathbb{M}^{n+1}(\bar{\kappa})$ uma paralela, com campo normal $\widetilde{N}^{\xi}$ dado por (1.9). Então, as curvaturas principais de $\widetilde{F}^{\xi}(M)$, com relação a $\widetilde{N}^{\xi}$, são

$$
\widetilde{\kappa}_{\imath}^{\xi}(x)=\frac{\bar{\kappa} s(\xi)+\kappa_{\imath}(x) c(\xi)}{c(\xi)-\kappa_{\imath}(x) s(\xi)}
$$

onde $\kappa_{\imath}(x)$ é a $\imath$-ésima curvatura principal de $F(M)$ em $x \in M$, para todo $\imath=1, \ldots, n$.

Além disso, se $\left\{e_{1}, \ldots, e_{n}\right\} \subset T_{x} M$ é uma base ortonormal de autovetores da segunda forma fundamental de $F(M)$, então

$$
\widetilde{g}_{x}^{\xi}\left(e_{\imath}, e_{\jmath}\right)=\left[c(\xi)-\kappa_{\imath}(x) s(\xi)\right]^{2} \delta_{\imath \jmath}
$$


Prova. Para cada ponto $x \in M$, escolha uma base ortonormal $\left\{e_{\imath}\right\}_{\imath=1}^{n} \in T_{x} M$ de autovetores da segunda forma fundamental $h$ de $F(M)$, assim ela satisfaz

$$
g_{x}\left(e_{\imath}, e_{\jmath}\right)=\delta_{\imath \jmath} \quad \text { e } \quad h_{x}\left(e_{\imath}, e_{\jmath}\right)=\kappa_{\imath}(x) \delta_{\imath \jmath}
$$

Tomando a diferencial das Equações 1.7 e 1.9, temos

$$
d \widetilde{F}_{x}^{\xi}\left(e_{\imath}\right)=c(\xi) d F_{x}\left(e_{\imath}\right)+s(\xi) d N_{x}\left(e_{\imath}\right)
$$

e

$$
d \widetilde{N}_{x}^{\xi}\left(e_{\imath}\right)=-\bar{\kappa} s(\xi) d F_{x}\left(e_{\imath}\right)+c(\xi) d N_{x}\left(e_{\imath}\right)
$$

Então

$$
\begin{aligned}
\widetilde{g}_{x}^{\xi}\left(e_{\imath}, e_{\jmath}\right)= & \left\langle d \widetilde{F}_{x}^{\xi}\left(e_{\imath}\right), d \widetilde{F}_{x}^{t}\left(e_{\jmath}\right)\right\rangle_{x} \\
= & \left\langle c(\xi) d F_{x}\left(e_{\imath}\right)+s(\xi) d N_{x}\left(e_{\imath}\right), c(\xi) d F_{x}\left(e_{\jmath}\right)+s(\xi) d N_{x}\left(e_{\jmath}\right)\right\rangle_{x} \\
= & c^{2}(\xi)\left\langle d F_{x}\left(e_{\imath}\right), d F_{x}\left(e_{\jmath}\right)\right\rangle_{x}+2 c(\xi) s(\xi)\left\langle d F_{x}\left(e_{\imath}\right), d N_{x}\left(e_{\jmath}\right)\right\rangle_{x} \\
& +s^{2}(\xi)\left\langle d N_{x}\left(e_{\imath}\right), d N_{x}\left(e_{\jmath}\right)\right\rangle_{x} \\
= & c^{2}(\xi) \delta_{\imath \jmath}-2 c(\xi) s(\xi) \kappa_{\imath}(x) \delta_{\imath \jmath}+s^{2}(\xi) \kappa_{\imath}^{2}(x) \delta_{\imath \jmath} \\
= & {\left[c(\xi)-\kappa_{\imath}(x) s(\xi)\right]^{2} \delta_{\imath \jmath} }
\end{aligned}
$$

$\mathrm{e}$

$$
\begin{aligned}
\widetilde{h}_{x}^{\xi}\left(e_{\imath}, e_{\jmath}\right)= & -\left\langle d \widetilde{F}_{x}^{\xi}\left(e_{\imath}\right), d \widetilde{N}_{x}^{\xi}\left(e_{\jmath}\right)\right\rangle_{x} \\
= & \left\langle c(\xi) d F_{x}\left(e_{\imath}\right)+s(\xi) d N_{x}\left(e_{\imath}\right), \bar{\kappa} s(\xi) d F_{x}\left(e_{\jmath}\right)-c(\xi) d N_{x}\left(e_{\jmath}\right)\right\rangle_{x} \\
= & \bar{\kappa} c(\xi) s(\xi)\left\langle d F_{x}\left(e_{\imath}\right), d F_{x}\left(e_{\jmath}\right)\right\rangle_{x} \\
& -\left(c^{2}(\xi)-\bar{\kappa} s^{2}(\xi)\right)\left\langle d F_{x}\left(e_{\imath}\right), d N_{x}\left(e_{\jmath}\right)\right\rangle_{x} \\
& -c(\xi) s(\xi)\left\langle d N_{x}\left(e_{\imath}\right), d N_{x}\left(e_{\jmath}\right)\right\rangle_{x} \\
= & \bar{\kappa} c(\xi) s(\xi) \delta_{\imath \jmath}+\left(c^{2}(\xi)-\bar{\kappa} s^{2}(\xi)\right) \kappa_{\imath}(x) \delta_{\imath \jmath}-c(\xi) s(\xi) \kappa_{\imath}^{2}(x) \delta_{\imath \jmath} \\
= & {\left[c(\xi)-\kappa_{\imath}(x) s(\xi)\right]\left[\bar{\kappa} s(\xi)+\kappa_{\imath}(x) c(\xi)\right] \delta_{\imath \jmath} . }
\end{aligned}
$$

Assim, concluímos que

$$
\widetilde{\kappa}_{\imath}^{\xi}(x)=\frac{\bar{\kappa} s(\xi)+\kappa_{\imath}(x) c(\xi)}{c(\xi)-\kappa_{\imath}(x) s(\xi)}
$$


Lema 1.16 Sejam $F: M^{n} \rightarrow \mathbb{M}^{n+1}(\bar{\kappa})$ uma hipersuperfície orientada, com campo vetorial normal unitário $N$ e $\widetilde{F}^{\xi}: M^{n} \rightarrow \mathbb{M}^{n+1}(\bar{\kappa})$ uma paralela, com campo normal $\widetilde{N}^{\xi}$ dado por (1.9). Então a curvatura média de $\widetilde{F}^{\xi}(M)$ é dado por

$$
\widetilde{H}^{\xi}(x)=\frac{\sum_{\jmath=0}^{n}(-1)^{\jmath} S_{\jmath}(x)\left[\bar{\kappa}(n-\jmath) c^{n-\jmath}(\xi) s^{\jmath+2}(\xi)-\jmath c^{n-\jmath+2}(\xi) s^{\jmath}(\xi)\right]}{\sum_{\jmath=0}^{n}(-1)^{\jmath} S_{\jmath}(x) c^{n-\jmath+1}(\xi) s^{\jmath+1}(\xi)},
$$

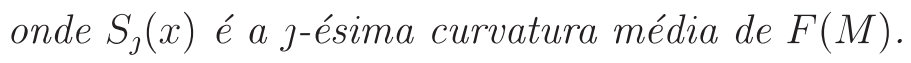

Prova Vamos provar o lema usando indução sobre $n$. Para $n=1$, como $\widehat{H}^{\xi}=\widehat{\kappa}_{1}^{\xi}(p)$ e $S_{1}(p)=\kappa_{1}$, a equação segue do Lema 1.15 .

Agora, suponha que a equação é verdadeira para $n-1$. Assim, para $n$ considere a imersão $F: M^{n} \rightarrow \mathbb{M}^{n+1}(\bar{\kappa})$.

Pela hipótese de indução, omitindo $x$ na equação, temos

$$
\sum_{\jmath=1}^{n-1} \widetilde{\kappa}_{\jmath}^{\xi}=\frac{\sum_{\jmath=0}^{n-1}(-1)^{\jmath} \sigma_{\jmath}\left(\kappa_{1}, \ldots, \kappa_{n-1}\right)\left[\bar{\kappa}(n-\jmath-1) c^{n-\jmath-1}(\xi) s^{\jmath+2}(\xi)-\jmath c^{n-\jmath+1}(\xi) s^{\jmath}(\xi)\right]}{\sum_{\jmath=0}^{n-1}(-1)^{\jmath} \sigma_{\jmath}\left(\kappa_{1}, \ldots, \kappa_{n-1}\right) c^{n-\jmath}(\xi) s^{\jmath+1}(\xi)}
$$

onde $\sigma_{\jmath}\left(\kappa_{1}, \ldots, \kappa_{n-1}\right)$ é o $\jmath$-ésimo polinômio simétrico elementar aplicado em $\left(\kappa_{1}, \ldots, \kappa_{n-1}\right)$. Assim

$$
\begin{aligned}
\widetilde{H}^{\xi}= & \sum_{\jmath=1}^{n} \widetilde{\kappa}_{j}^{\xi}=\sum_{\jmath=1}^{n-1} \widetilde{\kappa}_{j}^{\xi}+\widetilde{\kappa}_{n}^{\xi} \\
= & \frac{\sum_{\jmath=0}^{n-1}(-1)^{\jmath} \sigma_{\jmath}\left(\kappa_{1}, \ldots, \kappa_{n-1}\right)\left[\bar{\kappa}(n-\jmath-1) c^{n-\jmath-1}(\xi) s^{\jmath+2}(\xi)-\jmath c^{n-\jmath+1}(\xi) s^{\jmath}(\xi)\right]}{\sum_{\jmath=0}^{n-1}(-1)^{\jmath} \sigma_{\jmath}\left(\kappa_{1}, \ldots, \kappa_{n-1}\right) c^{n-\jmath}(\xi) s^{\jmath+1}(\xi)} \\
& +\frac{\bar{\kappa} s(\xi)+\kappa_{n} c(\xi)}{c(\xi)-\kappa_{n} s(\xi)} .
\end{aligned}
$$

Colocando no mesmo denominador e omitindo $\left(\kappa_{1}, \ldots, \kappa_{n-1}\right)$, temos que

$$
\begin{aligned}
\widetilde{H}^{\xi}= & \frac{\sum_{\jmath=0}^{n-1}(-1)^{\jmath} \sigma_{\jmath}\left[\bar{\kappa}(n-\jmath-1) c^{n-\jmath-1}(\xi) s^{\jmath+2}(\xi)-\jmath c^{n-\jmath+1}(\xi) s^{\jmath}(\xi)\right]\left[c(\xi)-\kappa_{n} s(\xi)\right]}{\sum_{\jmath=0}^{n-1}(-1)^{\jmath} \sigma_{\jmath} c^{n-\jmath}(\xi) s^{\jmath+1}(\xi)\left[c(\xi)-\kappa_{n} s(\xi)\right]} \\
& +\frac{\sum_{\jmath=0}^{n-1}(-1)^{\jmath} \sigma_{\jmath} c^{n-\jmath}(\xi) s^{\jmath+1}(\xi)\left[\bar{\kappa} s(\xi)+\kappa_{n} c(\xi)\right]}{\sum_{\jmath=0}^{n-1}(-1)^{\jmath} \sigma_{\jmath} c^{n-\jmath}(\xi) s^{\jmath+1}(\xi)\left[c(\xi)-\kappa_{n} s(\xi)\right]},
\end{aligned}
$$


reagrupando os termos

$$
\begin{aligned}
\widetilde{H}^{\xi}= & \frac{\sum_{\jmath=0}^{n-1}(-1)^{\jmath} \sigma_{\jmath}\left[\bar{\kappa}(n-\jmath) c^{n-\jmath}(\xi) s^{\jmath+2}(\xi)-\jmath c^{n+2-\jmath}(\xi) s^{\jmath}(\xi)\right]}{\sum_{\jmath=0}^{n-1}(-1)^{\jmath} \sigma_{\jmath} c^{n-\jmath+1}(\xi) s^{\jmath+1}(\xi)+\sum_{\jmath=0}^{n-1}(-1)^{\jmath+1} \sigma_{\jmath} \kappa_{n} c^{n-\jmath}(\xi) s^{\jmath+2}(\xi)} \\
& -\frac{\sum_{\jmath=0}^{n-1}(-1)^{\jmath} \sigma_{\jmath} \kappa_{n}\left[\bar{\kappa}(n-1-\jmath) c^{n-\jmath-1}(\xi) s^{\jmath+3}(\xi)-(\jmath+1) c^{n-\jmath+1}(\xi) s^{\jmath+1}(\xi)\right]}{\sum_{\jmath=0}^{n-1}(-1)^{\jmath} \sigma_{\jmath} c^{n-\jmath+1}(\xi) s^{\jmath+1}(\xi)+\sum_{\jmath=0}^{n-1}(-1)^{\jmath+1} \sigma_{\jmath} \kappa_{n} c^{n-\jmath}(\xi) s^{\jmath+2}(\xi)} .
\end{aligned}
$$

Renomeando os índices da segunda parcela da equação acima

$$
\begin{aligned}
\widetilde{H}^{\xi}= & \frac{\sum_{\jmath=0}^{n-1}(-1)^{\jmath} \sigma_{\jmath}\left[\bar{\kappa}(n-\jmath) c^{n-\jmath}(\xi) s^{\jmath+2}(\xi)-\jmath c^{n-\jmath+2}(\xi) s^{\jmath}(\xi)\right]}{\sum_{\jmath=0}^{n-1}(-1)^{\jmath} \sigma_{\jmath} c^{n-\jmath+1}(\xi) s^{\jmath+1}(\xi)+\sum_{\jmath=1}^{n}(-1)^{\jmath} \sigma_{\jmath-1} \kappa_{n} c^{n-\jmath+1}(\xi) s^{\jmath+1}(\xi)} \\
& -\frac{\sum_{\jmath=1}^{n}(-1)^{\jmath-1} \sigma_{\jmath-1} \kappa_{n}\left[\bar{\kappa}(n-\jmath) c^{n-\jmath}(\xi) s^{\jmath+2}(\xi)-\jmath c^{n-\jmath+2}(\xi) s^{\jmath}(\xi)\right]}{\sum_{\jmath=0}^{n-1}(-1)^{\jmath} \sigma_{\jmath} c^{n-\jmath+1}(\xi) s^{\jmath+1}(\xi)+\sum_{\jmath=1}^{n}(-1)^{\jmath} \sigma_{\jmath-1} \kappa_{n} c^{n-\jmath+1}(\xi) s^{\jmath+1}(\xi)} .
\end{aligned}
$$

Separando do somatório para $\jmath$ de 1 até $n-1$

$$
\begin{aligned}
\widetilde{H}^{\xi}= & \frac{\bar{\kappa} n c^{n}(\xi) s^{2}(\xi)+(-1)^{n+1} n \sigma_{n-1} \kappa_{n} c^{2}(\xi) s^{n}(\xi)}{c^{n+1}(\xi)+\sum_{j=1}^{n-1}(-1)^{\jmath}\left(\sigma_{\jmath}+\sigma_{\jmath-1} \kappa_{n}\right) c^{n-\jmath+1}(\xi) s^{\jmath+1}(\xi)+(-1)^{n} \sigma_{n-1} \kappa_{n} s^{n+1}(\xi)} \\
& -\frac{\sum_{\jmath=1}^{n-1}(-1)^{\jmath}\left(\sigma_{\jmath}+\kappa_{n} \sigma_{\jmath-1}\right)\left[\bar{\kappa}(n-\jmath) c^{n-\jmath}(\xi) s^{\jmath+2}(\xi)-\jmath c^{n-\jmath+2}(\xi) s^{\jmath}(\xi)\right]}{c^{n+1}(\xi)+\sum_{j=1}^{n-1}(-1)^{\jmath}\left(\sigma_{\jmath}+\sigma_{\jmath-1} \kappa_{n}\right) c^{n-\jmath+1}(\xi) s^{\jmath+1}(\xi)+(-1)^{n} \kappa_{n} \sigma_{n-1} s^{n+1}(\xi)}
\end{aligned}
$$

assim, pela Proposição 1.7, concluímos que

$$
\begin{aligned}
\widetilde{H}^{\xi}= & \frac{\left.\bar{\kappa} n c^{n}(\xi) s^{2}(\xi)+(-1)^{n+1} n S_{n} c^{2}(\xi) s^{n}(\xi)\right)}{c^{n+1}(\xi)+\sum_{j=1}^{n-1}(-1)^{\jmath} S_{\jmath} c^{n-\jmath+1}(\xi) s^{\jmath+1}(\xi)+(-1)^{n} S_{n} s^{n+1}(\xi)} \\
& -\frac{\sum_{\jmath=1}^{n-1}(-1)^{\jmath} S_{\jmath}\left[\bar{\kappa}(n-\jmath) c^{n-\jmath}(\xi) s^{\jmath+2}(\xi)-\jmath c^{n-\jmath+2}(\xi) s^{\jmath}(\xi)\right]}{c^{n+1}(\xi)+\sum_{j=1}^{n-1}(-1)^{\jmath} S_{\jmath} c^{n-\jmath+1}(\xi) s^{\jmath+1}(\xi)+(-1)^{n} S_{n} s^{n+1}(\xi)} \\
= & \frac{\sum_{\jmath=0}^{n}(-1)^{\jmath} S_{\jmath}\left[\bar{\kappa}(n-\jmath) c^{n-\jmath}(\xi) s^{\jmath+2}(\xi)-\jmath c^{n-\jmath+2}(\xi) s^{\jmath}(\xi)\right]}{\sum_{\jmath=0}^{n}(-1)^{\jmath} S_{\jmath} c^{n-\jmath+1}(\xi) s^{\jmath+1}(\xi)} .
\end{aligned}
$$

A seguir, apresentaremos um resultado devido a Cartan [6], que será usado para demostrar o Teorema 3.2.

Lema 1.17 (Cartan, 1938) Seja $\widetilde{F}^{\xi}: M^{n} \rightarrow \mathbb{M}^{n+1}(\bar{\kappa}), \xi \in(-\epsilon, \epsilon)$, uma família de hipersuperfícies paralela. Então, $F(M)$ é isoparamétrica se, e somente se, $\widetilde{H}^{\xi}(x)$ não depende de $x \in M$. 
Prova. Suponha que $F(M)$ é isoparamétrica, pelo Lema 1.15, temos que

$$
\widetilde{H}^{\xi}(x)=\sum_{\imath=1}^{n} \frac{\bar{\kappa} s(\xi)+\kappa_{\imath} c(\xi)}{c(\xi)-\kappa_{\imath} s(\xi)}
$$

onde $\kappa_{\imath}$ é a $\imath$-ésima curvatura principal de $F(M)$. Assim, $\widetilde{H}^{\xi}$ não depende de $x \in M$.

Reciprocamente, suponha que $\widetilde{H}^{\xi}(x)$ não depende de $x \in M$. Vamos provar por indução que todas as $l$-curvaturas médias $S_{l}$ de hipersuperfície $F(M)$ são constantes sobre $M$. Quando $l=1$, a afirmação segue da hipótese em $\xi=0$.

Agora, suponha que $S_{\jmath}(x)$ é constante sobre $M$, para todo $1 \leq \jmath \leq l$. Vamos provar que $S_{l+1}(x)$ também independe de $x$. Pelo Lema 1.16, temos que

$$
\sum_{\jmath=0}^{n}(-1)^{\jmath} S_{\jmath}(x) c^{n-\jmath}(\xi) s^{\jmath}(\xi)\left[\jmath c^{2}(\xi)-\bar{\kappa}(n-\jmath) s^{2}(\xi)+\widetilde{H}^{\xi} c(\xi) s(\xi)\right]=0 .
$$

Seja $V \in T M$ um campo diferencial sobre $M$. Tomando a diferencial da equação acima em relação a $V$ e usando a hipótese de indução, temos

$$
\sum_{\jmath=l+1}^{n}(-1)^{\jmath} d S_{\jmath}(V) c^{n-\jmath}(\xi) s^{\jmath}(\xi)\left[\jmath c^{2}(\xi)-\bar{\kappa}(n-\jmath) s^{2}(\xi)+\widetilde{H}^{\xi} c(\xi) s(\xi)\right]=0,
$$

dividindo por $s^{l+1}(\xi)$ e renomeando os índices

$$
\sum_{\jmath=1}^{n-l}(-1)^{l+\jmath} d S_{l+\jmath}(V) c^{n-l-\jmath}(\xi) s^{\jmath}(\xi)\left[(l+\jmath) c^{2}(\xi)-\bar{\kappa}(n-l-\jmath) s^{2}(\xi)+\widetilde{H}^{\xi} c(\xi) s(\xi)\right]=0
$$

em $\xi=0$, temos que $d S_{l+1}(V)=0$. Assim, todas as $l$-ésimas curvaturas médias de $F(M)$ são constantes, portanto as curvaturas principais são constantes, $\log O F(M)$ é isoparamétrica.

\subsection{Fluxo de Curvatura Média}

O Fluxo de Curvatura Média (FCM) evolui hipersuperfícies na direção normal com velocidade igual à curvatura média em cada ponto e é um fluxo do tipo gradiente para o funcional área [28], que se comporta como a equação do calor em um curto período de tempo. 
O FCM foi proposto por Mullins [29] em seu estudo sobre metais. O FCM e outros fluxos relacionados também foram estudados em varias áreas, como análise geométrica $[12,22]$, relatividade geral $[11,19]$, e topologia $[2,10]$.

Nesta seção, vamos apresentar alguns conceitos básicos sobre o fluxo de curvatura média em formas espaciais e mostrar a equivalência local entre as duas definições existentes na literatura.

Definição 1.18 Seja $F: M^{n} \rightarrow \mathbb{M}^{n+1}(\bar{\kappa})$ uma hipersuperfície orientada na forma espacial $\mathbb{M}^{n+1}(\bar{\kappa})$. Uma família a um parâmetro $\widehat{F}^{t}()=.\widehat{F}(., t)$ de hipersuperfícies $\widehat{F}$ : $M^{n} \times I \rightarrow \mathbb{M}^{n+1}(\bar{\kappa}), 0 \in I \subset \mathbb{R}$, é solução para o fluxo de curvatura média com condição inicial $F(M)$ se

$$
\left\{\begin{array}{l}
\frac{\partial}{\partial t} \widehat{F}(x, t)=\widehat{H}(x, t) \widehat{N}(x, t), \\
\widehat{F}(x, 0)=F(x),
\end{array}\right.
$$

onde $\widehat{H}^{t}()=.\widehat{H}(., t)$ é a curvatura média e $\widehat{N}^{t}()=.\widehat{N}(., t)$ é o campo vetorial normal unitário sobre $\widehat{F}^{t}(M)$.

Observação 1.19 Apesar da curvatura média $\widehat{H}^{\xi}(x)$ depender da escolha do campo normal unitário $\widehat{N}^{t}(x)$, o campo $\widehat{H}^{t}(x) \widehat{N}^{t}(x)$ é independente de tal escolha.

Uma hipersuperfície mínima é um exemplo trivial de solução para o FCM que se mantêm fixa ao longo do fluxo. As esferas e os cilindros são os mais fáceis, e na verdade, estão dentre os poucos exemplos não triviais explicitamente computáveis do FCM no espaço Euclidiano. Eles estão dentro de uma classe importante de soluções para o FCM, conhecidas como soluções auto-similares (veja [28]).

Exemplo 1.20 Seja $F: \mathbb{S}^{n} \rightarrow \mathbb{R}^{n+1}$ a imersão isométrica da esfera de raio $R_{0}$ no espaço Euclidiano, sem perda de generalidade podemos considerar que o centro de $F\left(\mathbb{S}^{n}\right)$ está na origem de $\mathbb{R}^{n+1}$.

Suponha que a solução para o fluxo de curvatura média é uma esfera centrada na origem para cada instante $t$, isto é,

$$
\widehat{F}(x, t)=\frac{R(t)}{R_{0}} F(x),
$$


onde $R(t)$ é o raio de $\widehat{F}^{t}\left(\mathbb{S}^{n}\right)$. Então a curvatura média de $\widehat{F}^{t}\left(\mathbb{S}^{n}\right)$ é dada por $\widehat{H}^{t}(x)=\frac{n}{R(t)}$ e o campo normal unitário por $\widehat{N}^{t}(x)=-\frac{1}{R_{0}} F(x)$. Por (1.13), temos

$$
\left\{\begin{array}{l}
R^{\prime}(t)=-\frac{n}{R(t)} \\
R(0)=R_{0}
\end{array}\right.
$$

Portanto, a solução da equação é

$$
\widehat{F}(x, t))=\frac{\sqrt{R_{0}^{2}-2 n t}}{R_{0}} F(x),
$$

para todo $t \in\left(-\infty, \frac{R_{0}^{2}}{2 n}\right)$.

Exemplo 1.21 Seja $F: \mathbb{S}^{m} \times \mathbb{R}^{n-m} \rightarrow \mathbb{R}^{m+1} \times \mathbb{R}^{n-m}=\mathbb{R}^{n+1}$ a imersão do cilindro de raio $R_{0}$ no espaço Euclidiano, definida por

$$
F\left(x_{1}, x_{2}\right)=\left(f\left(x_{1}\right), x_{2}\right)
$$

onde $f: \mathbb{S}^{m} \rightarrow \mathbb{R}^{m+1}$ é a imersão da esfera $m$-dimensional de raio $R_{0}$ no espaço Euclidiano $\mathbb{R}^{m+1}$ e $x_{2} \in \mathbb{R}^{n-m}$.

Suponha que a solução para o fluxo de curvatura média sobre $F\left(\mathbb{S}^{m} \times \mathbb{R}^{n-m}\right)$ é da forma

$$
\widehat{F}^{t}\left(x_{1}, x_{2}\right)=\left(\frac{R(t)}{R_{0}} f\left(x_{1}\right), x_{2}\right) .
$$

Então o campo normal em cada instante é dado por $\widehat{N}^{t}\left(x_{1}, x_{2}\right)=\left(-\frac{f\left(x_{1}\right)}{R_{0}}, 0\right)$ e a curvatura média é $\widehat{H}^{t}\left(x_{1}, x_{2}\right)=\frac{m}{R(t)}$. Nessas condições, a equação (1.13) é equivalente a

$$
\left\{\begin{array}{l}
R^{\prime}(t)=-\frac{m}{R(t)} \\
R(0)=R_{0}
\end{array}\right.
$$

Portanto, a solução para o fluxo é

$$
\widehat{F}^{t}\left(x_{1}, x_{2}\right)=\left(\frac{\sqrt{R_{0}^{2}-2 m t}}{R_{0}} f\left(x_{1}\right), x_{2}\right),
$$

para todo $t \in\left(-\infty, \frac{R_{0}^{2}}{2 m}\right)$. 
Proposição 1.22 ([28]) Se uma família de hipersuperfícies $\widetilde{F}: M \times I \rightarrow \mathbb{M}^{n+1}(\bar{\kappa})$, $I \subset \mathbb{R}$, satisfaz o sistema de equações diferenciais parciais

$$
\left\{\begin{array}{l}
\frac{\partial}{\partial t} \widetilde{F}(y, t)=\widetilde{H}(y, t) \widetilde{N}(y, t)+V(y, t), \\
\widetilde{F}(y, 0)=F(y)
\end{array}\right.
$$

onde $V(., t)$ é uma campo vetorial tangente a $\widetilde{F}^{t}(M)$, para cada $t \in I$. Então, na vizinhança de cada ponto do espaço e tempo, existe uma família de reparametrizações (suavemente dependendo do tempo) das aplicações $\widetilde{F}^{t}$ que satisfaz (1.13).

Se a variedade $M$ é compacta, podemos encontrar uma família de reparametrizações globais de $\widetilde{F}^{t}$ como acima, definida para todo $t \in I$, que não modifica a condição inicial $F(M)$ e satisfaz (1.13).

Inversamente, se $\widetilde{F}: M \times I \rightarrow \mathbb{M}^{n+1}(\bar{\kappa}), 0 \in I \subset \mathbb{R}$, é uma família de hipersuperfícies que admite uma reparametrização local para que seja uma solução do fluxo de curvatura média (1.13), então a aplicação $\widetilde{F}^{t}(y)$ satisfaz (1.15) para alguma família de campos $V(., t)$ tangente a $\widetilde{F}^{t}(M)$, para cada $t \in I$.

Prova. Primeiro vamos supor que $M$ é compacta, e construir uma família de reparametrizações globais que satisfaz a definição.

Pela hipótese de tangência, o campo vetorial dependente do tempo

$$
W(y, t)=-\left[d \widetilde{F}_{y}^{t}\right]^{-1}(V(y, t))
$$

é globalmente definido e suave. Seja $\varphi: M \times\left(\omega_{-}, \omega_{+}\right) \rightarrow M$ uma família de difeomorfismos de $M \operatorname{com} \varphi(x, 0)=x$, para todo $x \in M$, e

$$
\frac{\partial \varphi}{\partial t}(x, t)=W(\varphi(x, t), t)
$$

Esta família existe, é única e suave, pelo teorema de existência e unicidade de equações diferenciais ordinárias sobre a variedade compacta $M$. 
Considere a reparametrização $\widehat{F}(x, t)=\widetilde{F}(\varphi(x, t), t)$, temos

$$
\begin{aligned}
\frac{\partial \widehat{F}}{\partial t}(x, t)= & \frac{\partial \widetilde{F}}{\partial t}(\varphi(x, t), t)+d \widetilde{F}_{\varphi(x, t)}^{t}\left(\frac{\partial \varphi}{\partial t}(x, t)\right) \\
= & \widetilde{H}(\varphi(x, t), t) \widetilde{N}(\varphi(x, t), t)+V(\varphi(x, t), t)+d \widetilde{F}_{\varphi(x, t)}^{t}(W(\varphi(x, t), t)) \\
= & \widetilde{H}(\varphi(x, t), t) \widetilde{N}(\varphi(x, t), t)+V(\varphi(x, t), t) \\
& -d \widetilde{F}_{\varphi(x, t)}^{t}\left(\left[d \widetilde{F}_{\varphi(x, t)}^{t}\right]^{-1}(V(\varphi(x, t), t))\right) \\
= & \widetilde{H}(\varphi(x, t), t) \widetilde{N}(\varphi(x, t), t) \\
= & \widehat{H}(x, t) \widehat{N}(x, t) .
\end{aligned}
$$

Portanto, $\widehat{F}$ satisfaz o sistema (1.13) com condição inicial $\widehat{F}(x, 0)=F(x)$.

No caso não compacto, temos que trabalhar localmente no espaço e tempo. Resolvendo o sistema acima de equações diferenciais ordinárias em algum intervalo de tempo e um subconjunto aberto $\Omega \subset M$ com fecho compacto, então encontramos uma solução para o sistema (1.13) em um, possivelmente menor, subconjunto de $\Omega$ e algum intervalo de tempo.

Agora consideramos que a reparametrização $\widehat{F}(x, t)=\widehat{F}(\varphi(x, t), t)$ satisfaz (1.13). Derivando, temos

$$
\begin{aligned}
\frac{\partial \widehat{F}}{\partial t}(x, t) & =\frac{\partial \widetilde{F}}{\partial t}(\varphi(x, t), t)+d \widetilde{F}_{\varphi(x, t)}^{t}\left(\frac{\partial \varphi}{\partial t}(x, t)\right) \\
& =\widehat{H}(x, t) \widehat{N}(x, t) \\
& =\widetilde{H}(\varphi(x, t), t) \widetilde{N}(\varphi(x, t), t),
\end{aligned}
$$

isto é

$$
\frac{\partial \widetilde{F}}{\partial t}(y, t)=\widetilde{H}(y, t) \widetilde{N}(y, t)-d \widetilde{F}_{y}^{t}\left(\frac{\partial \varphi}{\partial t}\left(\varphi^{-1}(y, t), t\right)\right),
$$

para cada $x \in M$ e $t \in I$. Então, a ultima afirmação da proposição segue para $V(y, t)=$ $-d \widetilde{F}_{y}^{t}\left(\frac{\partial \varphi}{\partial t}\left(\varphi^{-1}(y, t)\right), t\right)$.

Corolário 1.23 Se uma familia de hipersuperfícies $\widehat{F}: M^{n} \times I \rightarrow \mathbb{M}^{n+1}(\bar{\kappa})$ satisfaz

$$
\left\langle\frac{\partial}{\partial t} \widehat{F}(x, t), \widehat{N}(x, t)\right\rangle=\widehat{H}(x, t),
$$


então ela pode ser localmente reparametrizada para uma solução do fluxo de curvatura média. Além disso, se $M$ é compacta, isto pode ser feito por uma reparametrização global, que não modifica a condição inicial.

Observação 1.24 A proposição e corolário anteriores dizem que a componente tangencial da velocidade da deformação não interfere na "forma" global durante o movimento.

Por esta propriedade de invariância vamos falar do fluxo de curvatura média considerado sobre subconjuntos de $\mathbb{M}^{n+1}(\bar{\kappa})$ e desconsiderando suas parametrizações. Damos então uma definição mais geométrica e alternativa para o fluxo de curvatura média.

Definição 1.25 Seja $F: M^{n} \rightarrow \mathbb{M}^{n+1}(\bar{\kappa})$ uma hipersuperfície orientada em uma forma espacial $\mathbb{M}^{n+1}(\bar{\kappa})$. Uma família a um parâmetro $\widehat{F}^{t}()=.\widehat{F}(., t)$ de hipersuperfícies $\widehat{F}$ : $M^{n} \times I \rightarrow \mathbb{M}^{n+1}(\bar{\kappa})$ é solução para o fluxo de curvatura média a menos de difeomorfismo de $M$ com condição inicial $F(M)$, se satisfaz a equação

$$
\left\{\begin{array}{l}
\left\langle\frac{\partial}{\partial t} \widehat{F}(x, t), \widehat{N}(x, t)\right\rangle=\widehat{H}(x, t), \\
\widehat{F}(x, 0)=F(x),
\end{array}\right.
$$

para todo $x \in M$ e $t \in I$.

O fluxo de curvatura média em curvas sobre superfícies, isto é, quando $n=1$ é conhecido na literatura como fluxo redutor de curvas. No plano Euclidiano temos o seguinte exemplo.

Exemplo 1.26 Considere a curva $X:(0, \pi) \rightarrow \mathbb{R}^{2}$ no plano Euclidiano, dado por, $X(s)=$ $(s,-\ln \cos (s))$. Vamos mostrar que

$$
\widehat{X}^{t}(s)=(s, t-\ln \cos (s))
$$

é solução para o fluxo redutor de curvas a menos de difeomorfismo, conhecida como Grim Reaper. O campo normal de $\widehat{X}^{t}$ é dado por $\widehat{N}^{t}(s)=(-\sin (s), \cos (s))$ e a curvatura por $\widehat{\kappa}^{t}(s)=\cos (s)$, portanto

$$
\left\langle\frac{\partial}{\partial t} \widehat{X}(s, t), N(s, t)\right\rangle=\langle(0,1),(-\sin (s), \cos (s))\rangle=\cos (s)=\widehat{\kappa}(s, t) .
$$

Observe que $\widehat{X}^{t}$ não é uma solução de (1.13). 
Uma classe importante de soluções para o fluxo de curvatura média, que contém o exemplo anterior, são os sólitons. Elas podem ser vistas como soluções estacionárias, no sentido geométrico, para o fluxo de curvatura média.

Definição 1.27 Seja $\widehat{F}^{t}: M^{n} \rightarrow \mathbb{M}^{n+1}(\bar{\kappa})$ uma solução para o fluxo de curvatura média a menos de difeomorfismo com condição inicial $F: M^{n} \rightarrow \mathbb{M}^{n+1}(\bar{\kappa})$. A condição inicial $F(M)$ é dito um sóliton do fluxo de curvatura média se

$$
\widehat{F}^{t}(x)=\phi^{t}(F(x))
$$

para todo $x \in M$, onde $\phi^{t}$ é uma família de isometrias de $\mathbb{M}^{n+1}(\bar{\kappa})$.

Observação 1.28 Como campos de Killing geram famílias de isometrias locais, esta definição é equivalente a definição dada por Hungerbühler e Smoczyk em [21]. 


\section{CAPÍTULO 2}

\section{Sólitons do Fluxo Redutor de Curvas na Esfera}

O fluxo de curvatura média sobre curvas de uma variedade Riemanniana bidimensional é conhecido como fluxo redutor de curvas (FRC). Para o estudo do FRC é importante conhecer as soluções autossimilares, que são modelos de singularidades do fluxo [20, 23].

As soluções autossimilares do FRC no plano são bem conhecidas. As duas soluções mais simples são as retas, que não são afetadas pelo fluxo, e os círculos que encolhem homoteticamente a um ponto em tempo finito. Uma outra solução simples é a curva Grim Reaper, gráfico da função $f(x)=\ln \cos (x)$ (veja Exemplo 1.26), ela é a única curva de translação do plano. Em [4], Altschuler mostrou uma curva que gira a uma velocidade constante denominada espiral yin-yang. Além dos círculos, existem outras curvas que encolhem homoteticamente ao longo do fluxo, elas foram classificadas por Abresch e Langer em [1]. Uma discussão sobre as curvas que expandem desta forma pode ser encontrado em [24, 35]. Finalmente, em [16] Halldorsson deu uma completa classificação das soluções autossimilares do FRC no plano Euclidiano.

Neste capítulo, vamos estudar os sólitons do fluxo redutor de curvas na esfera $\mathbb{S}^{2}$, que são um caso especial de soluções autossimilares. De acordo com a Definição 1.27, uma curva $X: I \rightarrow \mathbb{S}^{2} \subset \mathbb{R}^{3}$ é um sóliton do FRC na esfera se existe uma família de isometrias 
$A(t) \in O(3)$, tal que

$$
\widehat{X}^{t}(u)=A(t) X(u)
$$

é solução do fluxo de curvatura média a menos de difeomorfismo com condição inicial $X$, isto é, satisfaz (1.17).

Na Seção 2.1, vamos apresentar uma condição necessária e suficiente para que uma curva da esfera seja um sóliton do fluxo redutor de curvas. Na Seção 2.2, vamos usar esta condição para descrever os sólitons do FRC. Para finalizar, na Seção 2.3, vamos visualizar alguns exemplos destas curvas.

\subsection{Caracterização dos Sólitons do FRC na Esfera}

Nesta seção, caracterizaremos os sólitons do FRC na esfera como as curvas cuja curvatura é proporcional ao ângulo entre o vetor tangente e um vetor fixo de $\mathbb{R}^{3} \backslash\{0\}$.

Teorema 2.1 Seja $X: J \subset \mathbb{R} \rightarrow \mathbb{S}^{2} \subset \mathbb{R}^{3}$ uma curva na esfera. Então $X$ é um sóliton do fluxo redutor de curvas na esfera se, e somente se, existe um vetor $v \in \mathbb{R}^{3} \backslash\{0\}$, tal que

$$
\langle T, v\rangle=\kappa
$$

onde $T$ é o campo tangente unitário de $X$ e $\kappa$ sua curvatura.

Prova. Suponha, sem perda de generalidade, que $X(s)$ está parametrizada pelo comprimento de arco $s$. Se $X$ é um sóliton do FRC na esfera, temos que $\widehat{X}^{t}(s)=A(t) X(s)$ e $\widehat{X}^{t}$ satisfaz (1.17). Vamos escrever $A(t)=A_{1}(t) A_{2}(t) A_{3}(t)$, onde

$$
\begin{gathered}
A_{1}(t)=\left(\begin{array}{ccc}
1 & 0 & 0 \\
0 & \cos \left(\xi_{1}(t)\right) & -\sin \left(\xi_{1}(t)\right) \\
0 & \sin \left(\xi_{1}(t)\right) & \cos \left(\xi_{1}(t)\right)
\end{array}\right), A_{2}(t)=\left(\begin{array}{ccc}
\cos \left(\xi_{2}(t)\right) & 0 & \sin \left(\xi_{2}(t)\right) \\
0 & 1 & 0 \\
-\sin \left(\xi_{2}(t)\right) & 0 & \cos \left(\xi_{2}(t)\right)
\end{array}\right), \\
A_{3}(t)=\left(\begin{array}{ccc}
\cos \left(\xi_{3}(t)\right) & -\sin \left(\xi_{3}(t)\right) & 0 \\
\sin \left(\xi_{3}(t)\right) & \cos \left(\xi_{3}(t)\right) & 0 \\
0 & 0 & 1
\end{array}\right),
\end{gathered}
$$

e $\xi_{\imath}: I \rightarrow \mathbb{R}$ são funções reais, tais que $\xi_{\imath}(0)=0$, para $\imath=1,2,3$. 
Derivando (2.1), em $t=0$ temos

$$
\begin{aligned}
\frac{\partial}{\partial t} \widehat{X}(s, 0) & =A^{\prime}(0) X(s)=\left(A_{1}^{\prime}(0)+A_{2}^{\prime}(0)+A_{3}^{\prime}(0)\right) X(s) \\
& =\left[\xi_{1}^{\prime}(0)\left(\begin{array}{ccc}
0 & 0 & 0 \\
0 & 0 & -1 \\
0 & 1 & 0
\end{array}\right)+\xi_{2}^{\prime}(0)\left(\begin{array}{ccc}
0 & 0 & 1 \\
0 & 0 & 0 \\
-1 & 0 & 0
\end{array}\right)+\xi_{3}^{\prime}(0)\left(\begin{array}{ccc}
0 & -1 & 0 \\
1 & 0 & 0 \\
0 & 0 & 0
\end{array}\right)\right] X(s) \\
& =\left(\begin{array}{ccc}
0 & -\xi_{3}^{\prime}(0) & \xi_{2}^{\prime}(0) \\
\xi_{3}^{\prime}(0) & 0 & -\xi_{1}^{\prime}(0) \\
-\xi_{2}^{\prime}(0) & \xi_{1}^{\prime}(0) & 0
\end{array}\right) X(s) .
\end{aligned}
$$

Além disso, escrevendo $X(s)=\left(x_{1}(s), x_{2}(s), x_{3}(s)\right) \in \mathbb{S}^{2} \subset R^{3}$, lembramos que

$$
\begin{aligned}
\widehat{N}(s, 0) & =N(s)=T(s) \wedge X(s) \\
& =\left(x_{2}^{\prime}(s) x_{3}(s)-x_{2}(s) x_{3}^{\prime}(s),-x_{1}^{\prime}(s) x_{3}(s)+x_{1}(s) x_{3}^{\prime}(s), x_{1}^{\prime}(s) x_{2}(s)-x_{1}(s) x_{2}^{\prime}(s)\right) .
\end{aligned}
$$

Omitindo $s$ nas contas, temos

$$
\begin{aligned}
\left\langle\frac{\partial \widehat{X}}{\partial t}(s, 0), \widehat{N}(s, 0)\right\rangle= & \left\langle\left(-\xi_{3}^{\prime}(0) x_{2}+\xi_{2}^{\prime}(0) x_{3}, \xi_{3}^{\prime}(0) x_{1}-\xi_{1}^{\prime}(0) x_{3},-\xi_{2}^{\prime}(0) x_{1}+\xi_{1}^{\prime}(0) x_{2}\right),\right. \\
& \left.\left(x_{2}^{\prime} x_{3}-x_{2} x_{3}^{\prime},-x_{1}^{\prime} x_{3}+x_{1} x_{3}^{\prime}, x_{1}^{\prime} x_{2}-x_{1} x_{2}^{\prime}\right)\right\rangle \\
= & \xi_{1}^{\prime}(0)\left[x_{2}\left(x_{1}^{\prime} x_{2}-x_{1} x_{2}^{\prime}\right)-x_{3}\left(-x_{1}^{\prime} x_{3}+x_{1} x_{3}^{\prime}\right)\right] \\
& +\xi_{2}^{\prime}(0)\left[x_{3}\left(x_{2}^{\prime} x_{3}-x_{2} x_{3}^{\prime}\right)-x_{1}\left(x_{1}^{\prime} x_{2}-x_{1} x_{2}^{\prime}\right)\right] \\
& +\xi_{3}^{\prime}(0)\left[x_{1}\left(-x_{1}^{\prime} x_{3}+x_{1} x_{3}^{\prime}\right)-x_{2}\left(x_{2}^{\prime} x_{3}-x_{2} x_{3}^{\prime}\right)\right] \\
= & \xi_{1}^{\prime}(0)\left[x_{1}^{\prime}\left(x_{2}^{2}+x_{3}^{2}\right)-x_{1}\left(x_{2} x_{2}^{\prime}+x_{3} x_{3}^{\prime}\right)\right] \\
& +\xi_{2}^{\prime}(0)\left[x_{2}^{\prime}\left(x_{1}^{2}+x_{3}^{2}\right)-x_{2}\left(x_{1} x_{1}^{\prime}+x_{3} x_{3}^{\prime}\right)\right] \\
& +\xi_{3}^{\prime}(0)\left[x_{3}^{\prime}\left(x_{1}^{2}+x_{2}^{2}\right)-x_{3}\left(x_{1} x_{1}^{\prime}+x_{2} x_{2}^{\prime}\right)\right],
\end{aligned}
$$

como $x_{1}^{2}+x_{2}^{2}+x_{3}^{2}=1$ e $x_{1} x_{1}^{\prime}+x_{2} x_{2}^{\prime}+x_{3} x_{3}^{\prime}=0$,

$$
\begin{aligned}
\left\langle\frac{\partial \widehat{X}}{\partial t}(s, 0), \widehat{N}(s, 0)\right\rangle= & \xi_{1}^{\prime}(0)\left[x_{1}^{\prime}\left(1-x_{1}^{2}\right)-x_{1}\left(-x_{1} x_{1}^{\prime}\right)\right]+\xi_{2}^{\prime}(0)\left[x_{2}^{\prime}\left(1-x_{2}^{2}\right)-x_{2}\left(-x_{2} x_{2}^{\prime}\right)\right] \\
& +\xi_{3}^{\prime}(0)\left[x_{3}^{\prime}\left(1-x_{3}^{2}\right)-x_{3}\left(-x_{3} x_{3}^{\prime}\right)\right] \\
= & \xi_{1}^{\prime}(0) x_{1}^{\prime}+\xi_{2}^{\prime}(0) x_{2}^{\prime}+\xi_{3}^{\prime}(0) x_{3}^{\prime} \\
= & \left\langle\left(\xi_{1}^{\prime}(0), \xi_{2}^{\prime}(0), \xi_{3}^{\prime}(0)\right),\left(x_{1}^{\prime}, x_{2}^{\prime}, x_{3}^{\prime}\right)\right\rangle \\
= & \langle T, v\rangle,
\end{aligned}
$$


onde $v=\left(\xi_{1}^{\prime}(0), \xi_{2}^{\prime}(0), \xi_{3}^{\prime}(0)\right)$. Portanto, (1.17) em $t=0$, obtemos a equação (2.2) desejada.

Reciprocamente, seja $X(s)$ uma curva em $\mathbb{S}^{2} \subset \mathbb{R}^{3}$ parametrizada pelo comprimento do arco, tal que

$$
\langle T, v\rangle=\kappa
$$

para algum $v \in \mathbb{R}^{3} \backslash\{0\}$. A menos de isometria, podemos considerar que $v=a(0,0,1)=$ $a e_{3}$, para um $a>0$. Nestas condições, (2.2) é equivalente a

$$
a x_{3}^{\prime}(s)=k(s), \text { para todo } s \in I \text {. }
$$

Defina $\widehat{X}^{t}(s)=A(t) X(s)$, onde

$$
A(t)=\left(\begin{array}{ccc}
\cos (a t) & -\sin (a t) & 0 \\
\sin (a t) & \cos (a t) & 0 \\
0 & 0 & 1
\end{array}\right) .
$$

Vamos mostrar que $\widehat{X}^{t}$ é uma solução do fluxo redutor de curvas.

Observe que $\widehat{\kappa}^{t}(s)=\kappa(s)$ e $\widehat{N}^{t}(s)=A(t) N(s)$, para todo $t \in \mathbb{R}$. Desta forma, omitindo $s$ nas contas, temos que

$$
\begin{aligned}
\widehat{N}^{t}= & \left(\cos (a t)\left(x_{2}^{\prime} x_{3}-x_{2} x_{3}^{\prime}\right)-\sin (a t)\left(-x_{1}^{\prime} x_{3}+x_{1} x_{3}\right), \sin (a t)\left(x_{2}^{\prime} x_{3}-x_{2} x_{3}^{\prime}\right)\right. \\
& \left.+\cos (a t)\left(-x_{1}^{\prime} x_{3}+x_{1} x_{3}^{\prime}\right), x_{1}^{\prime} x_{2}-x_{1} x_{2}^{\prime}\right),
\end{aligned}
$$

Além disso, derivando $\widehat{X}^{t}$, obtemos

$$
\frac{\partial \widehat{X}}{\partial t}(s, t)=A^{\prime}(t) X(s)=a\left(-\sin (a t) x_{1}-\cos (a t) x_{2}, \cos (a t) x_{1}-\sin (a t) x_{2}, 0\right) .
$$


Portanto,

$$
\begin{aligned}
\left\langle\frac{\partial \widehat{X}}{\partial t}(s, t), \widehat{N}(s, t)\right\rangle= & a\left[\left(-\sin (a t) x_{1}-\cos (a t) x_{2}\right) \cos (a t)\left(x_{2}^{\prime} x_{3}-x_{2} x_{3}^{\prime}\right)\right. \\
& -\left(-\sin (a t) x_{1}-\cos (a t) x_{2}\right) \sin (a t)\left(-x_{1}^{\prime} x_{3}+x_{1} x_{3}^{\prime}\right) \\
& +\left(\cos (a t) x_{1}-\sin (a t) x_{2}\right) \sin (a t)\left(x_{2}^{\prime} x_{3}-x_{2} x_{3}^{\prime}\right) \\
& \left.+\left(\cos (a t) x_{1}-\sin (a t) x_{2}\right) \cos (a t)\left(-x_{1}^{\prime} x_{3}+x_{1} x_{3}^{\prime}\right)\right] \\
= & a\left[-x_{2}\left(x_{2}^{\prime} x_{3}-x_{2} x_{3}^{\prime}\right)+x_{1}\left(-x_{1}^{\prime} x_{3}+x_{1} x_{3}^{\prime}\right)\right] \\
= & a\left[x_{3}^{\prime}\left(x_{1}^{2}+x_{2}^{2}\right)-x_{3}\left(x_{1} x_{1}^{\prime}+x_{2} x_{2}^{\prime}\right)\right] \\
= & a\left[x_{3}^{\prime}\left(1-x_{3}^{2}\right)-x_{3}\left(-x_{3} x_{3}^{\prime}\right)\right] \\
= & a x_{3}^{\prime}=\kappa=\widehat{\kappa}^{t} .
\end{aligned}
$$

Intuitivamente $a$ é a velocidade angular com que o sóliton do FRC na esfera está girando ao longo do fluxo.

\subsection{Descrição dos Sólitons do FRC na Esfera}

Como vimos no Teorema 2.1, o estudo dos sólitons do fluxo redutor de curvas na esfera, se reduz a descrever todas as curvas que satisfazem (2.2), para algum vetor não nulo $v \in \mathbb{R}^{3}$.

Proposição 2.2 Seja $X: J \rightarrow \mathbb{S}^{2}$ uma curva parametrizada pelo comprimento de arco e $a>0$. Defina as funções

$$
\tau(s)=\left\langle T(s), e_{3}\right\rangle, \quad \nu(s)=\left\langle N(s), e_{3}\right\rangle \quad \text { e } \quad \alpha(s)=\left\langle X(s), e_{3}\right\rangle,
$$

onde $T$ e $N$ são os campos unitários tangente e normal a $X$, respectivamente. Então

$$
\kappa(s)=a \tau(s), \text { para todo } s \in J,
$$

se, e somente se, as funções $\tau, \nu$ e $\alpha$ satisfazem

$$
\left\{\begin{array}{l}
\tau^{\prime}=a \tau \nu-\alpha, \\
\nu^{\prime}=-a \tau^{2}, \\
\alpha^{\prime}=\tau .
\end{array}\right.
$$


com condições iniciais $(\tau(0), \nu(0), \alpha(0))$ satisfazendo $\tau^{2}(0)+\nu^{2}(0)+\alpha^{2}(0)=1$.

Prova. Suponha que $\kappa(s)=a \tau(s)$, para todo $s \in J$. Como $\tau, \nu$ e $\alpha$ satisfazem (1.3), substituindo (2.5) nesse sistema, temos (2.6).

Reciprocamente, suponha que as funções $\tau, \nu$ e $\alpha$ satisfazem (2.6). Igualando com (1.3), temos

$$
(a \tau-\kappa) \nu=0 \quad \text { e } \quad(a \tau-\kappa) \tau=0 .
$$

Como $\tau$ e $\nu$ se anulam simultaneamente apenas nos polos $e_{3} \mathrm{e}-e_{3}$, segue o resultado.

Agora vamos estudar o comportamento dos sólitons do FRC na esfera usando a Proposição 2.2. Para isso, vamos considerar os seguintes conjuntos, que podem ser visualizados na Figura 2.1.

- $\Gamma$ a geodésica de $\mathbb{S}^{2}$ ortogonal a $e_{3}$, denominada equador.

- $H_{\text {norte }}\left(\right.$ resp. $\left.H_{\text {sul }}\right)$ o hemisfério, sem sua fronteira, delimitado por $\Gamma$ que contém o ponto $e_{3}$ (resp. $-e_{3}$ ), denominado hemisfério norte (resp. hemisfério sul).

Observação 2.3 Seja $X(s)$ uma curva parametrizada pelo comprimento de arco $s$. As funções $\tau, \nu$ e $\alpha$, definidas em (2.4), têm as seguintes interpretações geométricas.

1. As funções $\tau, \nu$ e $\alpha$ são os cossenos dos ângulos entre $e_{3}$ e os vetores $T, N$ e $X$, respectivamente. Consequentemente, elas são limitadas por 1.

2. $\alpha$ é a função altura (com sinal) em relação a $e_{3}$.

3. $X(s) \in \Gamma$ se, e somente se, $\alpha(s)=0$.

4. $X(s) \in H_{\text {norte }}\left(\operatorname{resp} . X(s) \in H_{\text {sul }}\right)$ se, e somente se, $0<\alpha(s)($ resp. $0>\alpha(s))$.

5. Se $X$ satisfaz (2.5), então $|\kappa| \leq a$. 


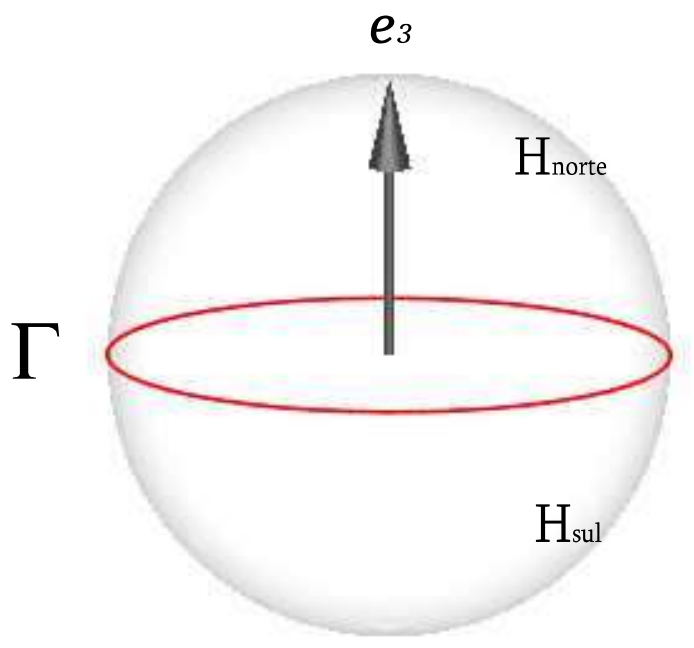

Figura 2.1: Esfera

A partir de agora, vamos usar a notação da Proposição 2.2 e provar uma série de lemas que permitirão a descrição dos sólitons do FRC da esfera no Teorema 2.17, no final desta seção.

A seguir, vamos deduzir uma identidade para as derivadas de ordem superior de $\alpha$, que será usada na demostração do Lema 2.5.

Lema 2.4 Seja $X: J \subset \mathbb{R} \rightarrow \mathbb{S}^{2}$ uma curva nas condições da Proposição 2.2. Se $\alpha^{(l)}(s)$ é a l-ésima derivada de $\alpha(s)=\left\langle X(s), e_{3}\right\rangle$, então as derivada de $\alpha(s)$ satisfazem

$$
\alpha^{(l+2)}(s)=a \sum_{\imath=0}^{l}\left(\begin{array}{l}
l \\
\imath
\end{array}\right) \alpha^{(l+1-\imath)}(s) \nu^{(\imath)}(s)-\alpha^{(l)}(s),
$$

para todo $l \geq 0$, onde $\left(\begin{array}{l}l \\ \imath\end{array}\right)=\frac{l !}{\imath !(l-\imath) !}$.

Prova. Vamos provar este lema por indução sobre $l$. Para $l=0$, derivando duas vezes $\alpha$ 
e usando (2.6), temos

$$
\begin{aligned}
\alpha^{\prime \prime}(s) & =\tau^{\prime}(s)=a \tau(s) \nu(s)-\alpha(s) \\
& =a \alpha^{\prime}(s) \nu(s)-\alpha(s)
\end{aligned}
$$

Agora suponha que (2.7) seja válida para $l$, assim

$$
\begin{aligned}
\alpha^{(l+3)}(s)= & \left(\alpha^{(l+2)}(s)\right)^{\prime} \\
= & \left(a \sum_{\imath=0}^{l}\left(\begin{array}{l}
l \\
\imath
\end{array}\right) \alpha^{(l+1-\imath)}(s) \nu^{(\imath)}(s)-\alpha^{(l)}(s)\right)^{\prime} \\
= & a \sum_{\imath=0}^{l}\left(\begin{array}{l}
l \\
\imath
\end{array}\right) \alpha^{(l+2-\imath)}(s) \nu^{(\imath)}(s)+a \sum_{\imath=0}^{l}\left(\begin{array}{l}
l \\
\imath
\end{array}\right) \alpha^{(l+1-\imath)}(s) \nu^{(\imath+1)}(s)-\alpha^{(l+1)}(s) \\
= & a \alpha^{(l+2)}(s) \nu(s)+a \sum_{\imath=1}^{l}\left(\begin{array}{l}
l \\
\imath
\end{array}\right) \alpha^{(l+2-\imath)}(s) \nu^{(\imath)}(s) \\
& +a \sum_{\imath=0}^{l-1}\left(\begin{array}{l}
l \\
\imath
\end{array}\right) \alpha^{(l+1-\imath)}(s) \nu^{(\imath+1)}(s)+a \alpha^{\prime}(s) \nu^{(l+1)}(s)-\alpha^{(l+1)}(s) .
\end{aligned}
$$

Renomeando os índices e agrupando os termos, temos

$$
\begin{aligned}
\alpha^{(l+3)}(s)= & a \alpha^{(l+2)}(s) \nu(s)+a \sum_{\imath=1}^{l}\left(\begin{array}{l}
l \\
\imath
\end{array}\right) \alpha^{(l+2-\imath)}(s) \nu^{(\imath)}(s) \\
& +a \sum_{\imath=1}^{l}\left(\begin{array}{c}
l \\
\imath-1
\end{array}\right) \alpha^{(l+2-\imath)}(s) \nu^{(\imath)}(s)+a \alpha^{\prime}(s) \nu^{(l+1)}(s)-\alpha^{(l+1)}(s) \\
= & a \alpha^{(l+2)}(s) \nu(s)+a \sum_{\imath=1}^{l}\left[\left(\begin{array}{l}
l \\
\imath
\end{array}\right)+\left(\begin{array}{c}
l \\
\imath-1
\end{array}\right)\right] \alpha^{(l+2-\imath)}(s) \nu^{(\imath)}(s) \\
& +a \alpha^{\prime}(s) \nu^{(l+1)}(s)-\alpha^{(l+1)}(s) .
\end{aligned}
$$

Como

$$
\begin{aligned}
\left(\begin{array}{l}
l \\
\imath
\end{array}\right)+\left(\begin{array}{c}
l \\
\imath-1
\end{array}\right) & =\frac{l !}{\imath !(l-\imath) !}+\frac{l !}{(\imath-1) !(l-(\imath-1)) !} \\
& =\frac{l !(l+1-\imath)+l ! \imath}{\imath !(l+1-\imath) !}=\frac{(l+1) !}{\imath !(l+1-\imath) !}=\left(\begin{array}{c}
l+1 \\
\imath
\end{array}\right)
\end{aligned}
$$

concluímos que

$$
\alpha^{(l+3)}=a \sum_{\imath=0}^{l+1}\left(\begin{array}{c}
l+1 \\
\imath
\end{array}\right) \alpha^{(l+2-\imath)}(s) \nu^{(\imath)}(s)-\alpha^{(l+1)}(s) .
$$


Lema 2.5 Seja $X: J \subset \mathbb{R} \rightarrow \mathbb{S}^{2}$ uma curva nas condições da Proposição 2.2. Se $s_{0}$ é um ponto critico de $\alpha(s)$, então

1. $X\left(s_{0}\right) \in \Gamma$ se, e somente se, $s_{0}$ é ponto de inflexão de $\alpha$.

2. $X\left(s_{0}\right) \in H_{\text {norte }}\left(r e s p . X\left(s_{0}\right) \in H_{\text {sul }}\right.$ ) se, e somente se, $s_{0}$ é ponto de máximo (resp. minimo) local de $\alpha$.

Prova. Para o item 1 , se $s_{0}$ é ponto de inflexão, temos $\alpha^{\prime \prime}\left(s_{0}\right)=0$. Pelo lema anterior, segue que $\alpha\left(s_{0}\right)=0$. Logo, pela Observação 2.3, $X\left(s_{0}\right) \in \Gamma$.

Agora, supondo que $X\left(s_{0}\right) \in \Gamma$, vamos provar por indução em $l$ que $\alpha^{(l)}\left(s_{0}\right)=0$, para todo $l$. Como $\alpha\left(s_{0}\right)=\alpha^{\prime}\left(s_{0}\right)=0$, pelo lema anterior, isso implica que $\alpha^{\prime \prime}\left(s_{0}\right)=0$. Suponha que $\alpha^{(\imath)}\left(s_{0}\right)=0$, para todo $\imath=1, \ldots, l+1$, pelo Lema 2.4 , segue que $\alpha^{(l+2)}\left(s_{0}\right)=0$. Assim, todas as derivadas de $\alpha$ se anulam em $s_{0}$. Isso implica que $s_{0}$ é um ponto de inflexão de $\alpha$.

Para o item 2, considerando $s=s_{0}$ e $\imath=0$ em (2.7), temos que $\alpha^{\prime \prime}\left(s_{0}\right)=-\alpha\left(s_{0}\right)$. Portanto, pela Observação 2.3, segue o resultado.

Lema 2.6 Seja $X: J \subset \mathbb{R} \rightarrow \mathbb{S}^{2}$ uma curva nas condições da Proposição 2.2. Se $s_{0}$ é um ponto critico de $\alpha(s)$, então $X\left(s_{0}\right) \in H_{\text {norte }}$ (resp. $H_{\text {sul }}$ ) se, e somente se, em uma vizinhança de $X\left(s_{0}\right)$ a curva $X$ está abaixo (resp. acima) do paralelo (circulo de $\mathbb{S}^{2}$ ortogonal $\left.a e_{3}\right)$ que passa por $X\left(s_{0}\right)$. Além disso, $X$ não tangencia $\Gamma$ em $s_{0}$.

Prova. Como $\alpha$ é a função altura de $X$, temos que $X$ está abaixo (resp. acima) do paralelo que passa por $X\left(s_{0}\right)$ se, e somente se, $s_{0}$ é um ponto de máximo (resp. minimo) local de $\alpha$. Portanto, pelo Lema 2.5 item 2, segue a primeira afirmação.

Agora suponha que $X$ tangencia $\Gamma$ em $s_{0}$, então $s_{0}$ é um ponto de máximo ou minimo local de $\alpha$, contradizendo o Lema 2.5 item 1. 
Lema 2.7 Seja $X: J \subset \mathbb{R} \rightarrow \mathbb{S}^{2}$ uma curva nas condições da Proposição 2.2. Se $s_{0}$ é um ponto crítico de $\tau(s)=\left\langle T(s), e_{3}\right\rangle$, então $s_{0}$ é um ponto de mínimo (resp. máximo) local de $\tau$ se, e somente se, $\tau\left(s_{0}\right)<0$ (resp. $\left.\tau\left(s_{0}\right)>0\right)$.

Prova. Derivando duas vezes $\tau$ e usando (2.6) em $s=s_{0}$, temos

$$
\begin{aligned}
\tau^{\prime \prime}\left(s_{0}\right) & =a \tau^{\prime}\left(s_{0}\right) \nu\left(s_{0}\right)+a \tau\left(s_{0}\right) \nu^{\prime}\left(s_{0}\right)-\alpha^{\prime}\left(s_{0}\right) \\
& =a \tau\left(s_{0}\right) \nu^{\prime}\left(s_{0}\right)-\alpha^{\prime}\left(s_{0}\right) \\
& =-a^{2} \tau^{3}\left(s_{0}\right)-\tau\left(s_{0}\right) \\
& =-\tau\left(s_{0}\right)\left(a^{2} \tau^{2}\left(s_{0}\right)+1\right) .
\end{aligned}
$$

Portando, $\tau^{\prime \prime}\left(s_{0}\right)>0\left(\operatorname{resp} . \tau^{\prime \prime}\left(s_{0}\right)<0\right)$ se, e somente se, $\tau\left(s_{0}\right)<0\left(\operatorname{resp} . \tau\left(s_{0}\right)>0\right)$.

Lema 2.8 Seja $X: J \subset \mathbb{R} \rightarrow \mathbb{S}^{2}$ uma curva nas condições da Proposição 2.2, então todo ponto critico de $\nu(s)=\left\langle N(s), e_{3}\right\rangle$ é um ponto de inflexão e $\nu$ é não crescente.

Prova. Seja $s_{0}$ um ponto critico de $\nu$, por $(2.6), \tau\left(s_{0}\right)=0$. Derivando $\nu^{\prime}$ e fazendo $s=s_{0}$, temos

$$
\nu^{\prime \prime}\left(s_{0}\right)=-2 a \tau\left(s_{0}\right) \tau^{\prime}\left(s_{0}\right)=0 .
$$

Por (2.6), derivando $\nu^{\prime \prime}$ e fazendo $s=s_{0}$, obtemos

$$
\begin{aligned}
\nu^{\prime \prime \prime}\left(s_{0}\right) & =-2 a\left(\tau^{\prime}\left(s_{0}\right)\right)^{2}-2 a \tau\left(s_{0}\right) \tau^{\prime \prime}\left(s_{0}\right) \\
& =-2 a\left(a \tau\left(s_{0}\right)-\alpha\left(s_{0}\right)\right)^{2} \\
& =-2 a \alpha^{2}\left(s_{0}\right) .
\end{aligned}
$$

Assim, se $\alpha\left(s_{0}\right) \neq 0$, temos que $\nu^{\prime}\left(s_{0}\right)=\nu^{\prime \prime}\left(s_{0}\right)=0$ e $\nu^{\prime \prime \prime}\left(s_{0}\right) \neq 0$. Portanto, $s_{0}$ é ponto de inflexão de $\nu$. Se $\alpha\left(s_{0}\right)=0$, temos que $\alpha\left(s_{0}\right)=\alpha^{\prime}\left(s_{0}\right)=\alpha^{\prime \prime}\left(s_{0}\right)=0$. Pelo Lema 2.4, as derivadas $\alpha^{(\imath)}\left(s_{0}\right)=0$, para todo $\imath \in \mathbb{N}$. Como $\nu^{\prime}=-a \tau^{2}=-a\left(\alpha^{\prime}\right)^{2}$, todas as derivadas de $\nu$ podem ser escritas como soma de potencias das derivadas de $\alpha$. Portanto, todas as derivadas de $\nu$ se anulam em $s=s_{0}$ e $s_{0}$ é ponto de inflexão de $\nu$.

Além disso, por (2.6), $\nu^{\prime}(s) \leq 0$ para todo $s \in \mathbb{R}$ e $\nu$ é não crescente. 
Por substituição direta em (2.6), temos a seguintes equivalências.

Observação 2.9 Seja $X: J \subset \mathbb{R} \rightarrow \mathbb{S}^{2}$ uma curva nas condições da Proposição 2.2, então as seguintes afirmações são equivalentes:

1. $\tau\left(s_{0}\right)=0$,

2. $\nu^{\prime}\left(s_{0}\right)=0$,

3. $\alpha^{\prime}\left(s_{0}\right)=0$.

Nem sempre o sóliton intersecta duas vezes o equador, mas quando isso ocorre a curva se comporta de forma simples entre dois pontos consecutivos de intersecção, como vamos demostrar no seguinte lema.

Lema 2.10 Seja $X: J \subset \mathbb{R} \rightarrow \mathbb{S}^{2}$ uma curva nas condições da Proposição 2.2, então entre dois pontos consecutivos em que $X$ intersecta o equador existe um único ponto critico $s_{0}$ da função altura $\alpha(s)$. Além disso, $\kappa\left(s_{0}\right)=0$.

Prova. Sejam $s_{1}<s_{2}$ dois pontos em que $X$ intersecta $\Gamma$. Pela Observação 2.3 item $1, \alpha\left(s_{1}\right)=\alpha\left(s_{2}\right)=0$ e $\alpha \neq 0$, para todo $s \in\left(s_{1}, s_{2}\right)$. Assim, pelo Teorema do valor médio (veja [26]), existe $s_{0} \in\left(s_{1}, s_{2}\right)$ tal que $\alpha^{\prime}\left(s_{0}\right)=0$. Como $\alpha$ tem sinal definido em $\left(s_{1}, s_{2}\right)$, pelo Lema 2.5 , os pontos críticos de $\alpha$ em $\left(s_{1}, s_{2}\right)$ são todos de máximo ou todos de mínimo, dependendo se $X\left(\left(s_{1}, s_{2}\right)\right)$ esta contido em $H_{\text {sul }}$ ou $H_{\text {norte }}$, respectivamente. Portanto, existe um único ponto crítico de $\alpha$ em $\left(s_{1}, s_{2}\right)$.

Lema 2.11 Seja $X: J \subset \mathbb{R} \rightarrow \mathbb{S}^{2}$ uma curva nas condições da Proposição 2.2. Se $s_{1}<s_{2}$ são pontos críticos de $\alpha$, tais que $0<\nu\left(s_{\imath}\right)$ (resp. $0>\nu\left(s_{\imath}\right)$ ), para $\imath=1$, 2, então $\left|\alpha\left(s_{1}\right)\right|<\left|\alpha\left(s_{2}\right)\right|\left(\right.$ resp. $\left.\left|\alpha\left(s_{1}\right)\right|>\left|\alpha\left(s_{2}\right)\right|\right)$.

Prova. Defina $f(s)=\sqrt{1-\nu^{2}(s)}$, como $\nu$ é não crescente, está função é não crescente no intervalo onde $\nu$ é positiva e não decrescente onde $\nu$ é positiva. 


$$
\begin{gathered}
\text { Como } \tau^{2}(s)+\nu^{2}(s)+\alpha^{2}(s)=1 \text {, em } s=s_{\imath} \text {, para } \imath=1,2, \text { temos } \\
\left|\alpha\left(s_{\imath}\right)\right|=\sqrt{1-\nu^{2}\left(s_{\imath}\right)}=f\left(s_{\imath}\right) .
\end{gathered}
$$

Assim, se $\nu\left(s_{\imath}\right)<0\left(\right.$ resp. $\left.\nu\left(s_{\imath}\right)<0\right)$, temos

$$
\left|\alpha\left(s_{1}\right)\right|=f\left(s_{1}\right)<f\left(s_{2}\right)=\left|\alpha\left(s_{2}\right)\right|
$$

$\left(\right.$ resp. $\left.\left|\alpha\left(s_{1}\right)\right|=f\left(s_{1}\right)>f\left(s_{2}\right)=\left|\alpha\left(s_{2}\right)\right|\right)$.

A seguir, vamos apresentar o Lema de Barbalat, cuja demostração pode ser encontrada em [32]. Este é um resultado clássico da analise da reta que será utilizado para demostrar o Lema 2.13.

Lema 2.12 (Lema de Barbalat, [32]) Seja $f:\left(s_{0},+\infty\right) \subset \mathbb{R} \rightarrow \mathbb{R}$ uma função diferenciável, tal que existe $\lim _{s \rightarrow+\infty} f(s)$ e $f^{\prime}(s)$ é uniformemente contínua. Então

$$
\lim _{s \rightarrow+\infty} f^{\prime}(s)=0
$$

Lema 2.13 Seja $X: J \subset \mathbb{R} \rightarrow \mathbb{S}^{2}$ uma curva nas condições da Proposição 2.2, então $X(s)$ assintota $\Gamma$ quando $t \rightarrow \pm \infty$.

Prova. Pela Observação 2.3 item 3, basta provar que $\lim _{s \rightarrow \pm \infty} \alpha(s)=0$. Vamos começar provando que

$$
\lim _{s \rightarrow \pm \infty} \tau(s)=0 .
$$

Como $\nu$ é não crescente e limitada, temos que existe o limite de $\nu$ quando $s \rightarrow \pm \infty$. Além disso,

$$
\left|\nu^{\prime \prime}\right|=\left|-2 a \tau \tau^{\prime}\right|=|2 a \tau(a \tau \nu-\alpha)| \leq 2 a(a+1),
$$

$\operatorname{assim} \nu^{\prime}$ é uma função uniformemente contínua. Desta maneira, pelo Lema 2.12,

$$
\lim _{s \rightarrow \pm \infty} \nu^{\prime}(s)=0
$$

Como $\nu^{\prime}(s)=-a \tau^{2}(s)$, temos $(2.8)$. 
Agora, vamos provar que

$$
\lim _{s \rightarrow \pm \infty} \tau^{\prime}(s)=0
$$

Derivando duas vezes $\tau$ por (2.6), temos

$$
\begin{aligned}
\tau^{\prime \prime} & =a \tau^{\prime} \nu+a \tau \nu^{\prime}-\alpha^{\prime} \\
& =a(a \tau \nu-\alpha) \nu-a^{2} \tau^{3}-\tau \\
& \leq 2 a^{2}+a+1 .
\end{aligned}
$$

Isto implica que $\tau^{\prime}$ é uniformemente contínua. Assim, pelo Lema 2.12 e (2.8), segue (2.9).

Portanto, por (2.6), (2.8), (2.9) e como $\nu$ é limitado, temos que

$$
\lim _{s \rightarrow \pm \infty} \alpha(s)=\lim _{s \rightarrow \pm \infty}\left(a \tau(s) \nu(s)-\tau^{\prime}(s)\right)=0 .
$$

Lema 2.14 Seja $X: J \subset \mathbb{R} \rightarrow \mathbb{S}^{2}$ uma curva nas condições da Proposição 2.2. Se existe $s_{0} \in \mathbb{R}$, tal que $X\left(\left(s_{0},+\infty\right)\right) \subset H_{\text {norte }}$ (resp. $\left.X\left(\left(s_{0},+\infty\right)\right) \subset H_{\text {sul }}\right)$, então existe um $s_{*}>s_{0}$, tal que $\alpha$ é decrescente (rep. crescente) em $\left(s_{*},+\infty\right)$.

Prova. Primeiro, observe que existe no máximo um ponto critico de $\alpha$ em $\left(s_{0},+\infty\right)$. De fato, suponha que existam dois pontos críticos $s_{1}, s_{2}$ de $\alpha$ em $\left(s_{0},+\infty\right)$. Pelo Lema 2.4, estes dois pontos são de máximo (resp. mínimo), assim existe um ponto de mínimo (resp. máximo) de $\alpha$ entre $s_{1}$ e $s_{2}$, contradizendo assim o Lema 2.4. Assim, existe um $s_{*} \in\left(s_{0},+\infty\right)$ tal que $\alpha$ é estritamente monótona em $\left(s_{*},+\infty\right)$. Como a função $\alpha$ é positiva (resp. negativa) neste intervalo e seu limite vai para zero quando $s \rightarrow+\infty$, temos que $\alpha$ é decrescente (resp. crescente) neste intervalo.

De forma análoga, temos o seguinte lema.

Lema 2.15 Seja $X: J \subset \mathbb{R} \rightarrow \mathbb{S}^{2}$ uma curva nas condições da Proposição 2.2. Se existe $s_{0} \in \mathbb{R}$, tal que $X\left(\left(-\infty, s_{0}\right)\right) \subset H_{\text {norte }}$ (resp. $\left.X\left(\left(-\infty, s_{0}\right)\right) \subset H_{\text {sul }}\right)$, então existe um $s_{*}<s_{0}$, tal que $\alpha$ é crescente (rep. decrescente) em $\left(-\infty, s_{*}\right)$. 
A seguir, vamos estudar os autovalores dos pontos críticos do campo de vetores sobre a esfera unitária definido por (2.6). Isto nos permite compreender o comportamento das curvas integrais deste campo.

Lema 2.16 Seja $(\tau, \nu, \alpha)$ uma solução de (2.6), com condição inicial $\tau^{2}(0)+\nu^{2}(0)+$ $\alpha^{2}(0)=1$. Considere o campo $\psi: \mathbb{S}^{2} \rightarrow T \mathbb{S}^{2} \subset \mathbb{R}^{3}$ dado por

$$
\psi(\tau, \nu, \alpha)=\left(a \tau \nu-\alpha,-a \tau^{2}, \tau\right)
$$

Então os pontos singulares de $\psi$ são $\pm e_{2}=(0, \pm 1,0)$ e os autovalores de $d \psi_{e_{2}}$ (resp. $\left.d \psi_{-e_{2}}\right) s \tilde{a} o$

$$
\lambda=\frac{a \pm \sqrt{a^{2}-4}}{2}\left(\operatorname{resp} . \lambda=\frac{-a \pm \sqrt{a^{2}-4}}{2}\right) .
$$

Assim, se $0<a<2$ os autovalores de $\psi$ são complexos e se $a \geq 2$ os autovalores de $\psi$ são reais.

Prova. Calculando a diferencial de $\psi$, temos

$$
d \psi=\left(\begin{array}{ccc}
a \nu & a \tau & -1 \\
-2 a \tau & 0 & 0 \\
1 & 0 & 0
\end{array}\right)
$$

Aplicando no ponto $e_{2}$, temos

$$
d \psi_{e_{2}}=\left(\begin{array}{ccc}
a & 0 & -1 \\
0 & 0 & 0 \\
1 & 0 & 0
\end{array}\right)
$$

Portanto, $\lambda \in \mathbb{R}$ é autovalor de $d \psi_{e_{2}}$ se existe $v \in T_{e_{2}} \mathbb{S}^{2}=\left\{v=\left(v_{1}, 0, v_{3}\right) / v_{1}, v_{3} \in \mathbb{R}\right\}$, tal que

$$
d \psi_{e_{2}}(v)=\left(\begin{array}{ccc}
a & 0 & -1 \\
0 & 0 & 0 \\
1 & 0 & 0
\end{array}\right)\left(\begin{array}{c}
v_{1} \\
0 \\
v_{3}
\end{array}\right)=\lambda\left(v_{1}, 0, v_{3}\right)
$$

isto implica que,

$$
\left\{\begin{array}{l}
a v_{1}-v_{3}=\lambda v_{1}, \\
v_{1}=\lambda v_{3},
\end{array}\right.
$$


$\operatorname{logo} \lambda^{2}-a+1=0$, onde concluímos que

$$
\lambda=\frac{a \pm \sqrt{a^{2}-4}}{2}
$$

Para $-e_{2}$ o resultado segue de forma análoga.

Quando $0<a<2$ os autovalores da diferencial do campo $\psi$ em seus pontos singulares são complexos. Assim, as curvas integrais saem de $-e_{2}$ e vão para $e_{2}$ em forma de espiral, intersectando a geodésica $\alpha=0$ infinitas vezes. Quando $a \geq 2$, os autovalores são reais não nulos e as curvas integrais saem de $-e_{2}$ e chegam em $e_{2}$ de forma "retilínea" quando estão suficientemente próximos destes pontos, como podemos ver na Figura 2.2.

A seguir, vamos apresentar o retrato de fase do campo $\psi$ definido por (2.10) sobre a esfera unitária, que nos permite compreender melhor o comportamento das funções $\tau, \nu$ e $\alpha$ e consequentemente o comportamento dos sólitons. As curvas integrais estão representadas de azul, a geodésica $\alpha=0$ de vermelho e a geodésica $\tau=0$ de verde.

O retrato de fase de $\psi$, para $a=1,2$ e 3, podem ser vistos na Figura 2.2.
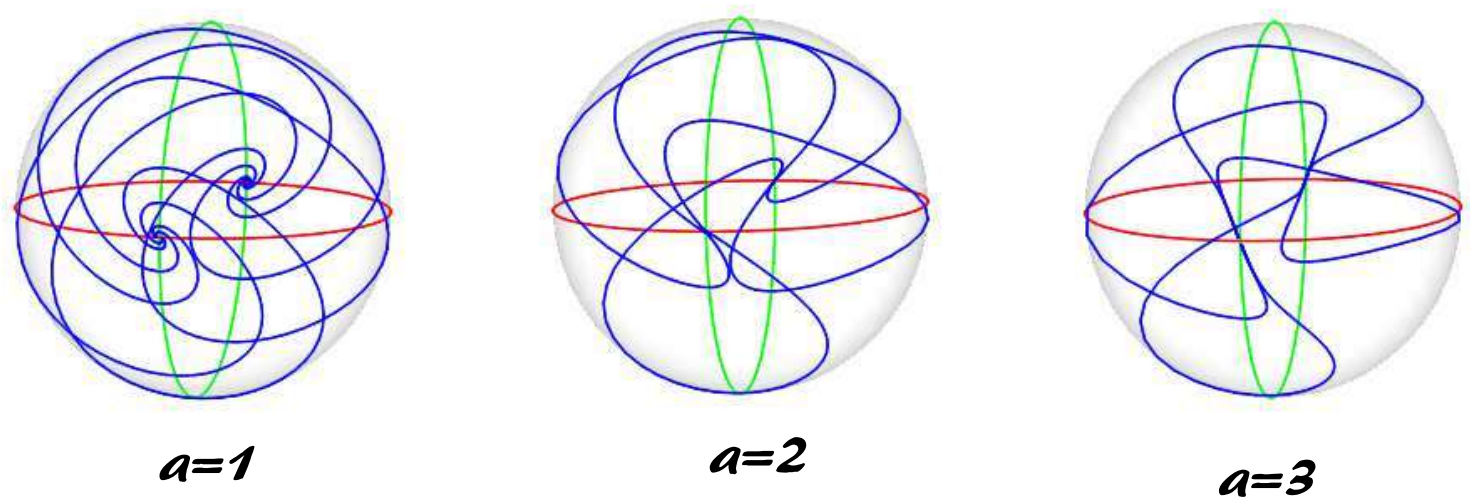

Figura 2.2: Retrato de Fase de $\psi$

Teorema 2.17 Para cada $v \in \mathbb{R}^{3}$ não nulo, existe uma família a um parâmetro de sólitons do fluxo redutor de curvas na esfera $X: \mathbb{R} \rightarrow \mathbb{S}^{2}$, definida para toda a reta $\mathbb{R}$. Os 
dois fins de cada curva são assintóticos a mesma geodésica $\Gamma$ ortogonal a $v$. Entre dois pontos consecutivos em que $X$ intersecta $\Gamma$, caso existam, a função altura $\alpha(s)=\left\langle X(s), \frac{v}{\|v\|}\right\rangle$ tem um único ponto critico $s_{o}$, que é ponto de máximo (resp. mínimo) local se $\alpha\left(s_{o}\right)>0$ (resp. $\alpha\left(s_{o}\right)<0$. Além disso,

- Se $0<\|v\|<2, X$ intersecta $\Gamma$ em infinitos pontos e a norma $|\alpha|$ nos pontos críticos de a forma uma sequência que é estritamente crescente quando $\nu(s)=$ $\left\langle N(s), \frac{v}{\|v\|}\right\rangle>0$ e estritamente decrescente quando $\nu(s)<0$.

- $S e\|v\| \geq 2, X$ intersecta $\Gamma$ no máximo em um número finito de pontos e cada um dos fins converge para $\Gamma$ sem se auto intersectar.

Prova. Sem perda de generalidade, podemos supor que $v=a e_{3}$, onde $a>0$. Assim, usando a notação da Proposição 2.2, temos que $\kappa(s)=a \tau(s)$ e as funções $\tau, \nu$ e $\alpha$ satisfazem (2.6).

Este sistema define o campo

$$
\psi(\tau, \nu, \alpha)=\left(a \tau \nu-\alpha,-a \tau^{2}, \tau\right)
$$

sobre a esfera unitária. Considerando o retrato de fase de $\psi$, cada curva integral de $\psi$ corresponde a um sóliton do FRC. Assim, para cada $a>0$, temos uma família a um parâmetro destas curvas. Como $\psi$ é um campo completo, cada sóliton está definida para todo $s \in \mathbb{R}$. Além disso, pelo Lema 2.13, cada um dos fins de $X$ assíntota $\Gamma$ quando $s \rightarrow \pm \infty$.

Pelo Lema 2.10, entre dois pontos em que $\mathrm{X}$ intersecta $\Gamma$ a função altura $\alpha$ possui um, e somente um, ponto critico, que é de máximo local se atingido no hemisfério norte e de mínimo local se atingido no hemisfério sul.

Se $0<a<2$, pelo Lema 2.16, $X$ intersecta $\Gamma$ infinitas vezes. Além disso, pelo Lema 2.11, a norma $|\alpha|$ aplicada nos pontos críticos de $\alpha$ forma uma sequência que é estritamente crescente enquanto $\nu$ é positivo e estritamente decrescente enquanto $\nu$ é negativo. Observe que, por (2.6) $\nu$ é estritamente decrescente e pelo Lema 2.13

$$
\lim _{s \rightarrow \pm \infty} \nu(s)=\mp 1
$$


portanto $\nu$ possui um, e somente um, zero.

Se $a \geq 2$, pelo Lema 2.16, existem $s_{-}, s_{+} \in \mathbb{R}$, tal que $\alpha$ tem no máximo um número finito de zeros nos intervalos $\left(-\infty, s_{-}\right]$e $\left[s_{+},+\infty\right)$. Suponha que $\alpha$ tem uma infinidade de zeros em $\left[s_{-}, s_{+}\right]$, isto é, existem

$$
s_{-} \leq s_{1}<s_{2}<\ldots<s_{n}<\ldots \leq s_{+}
$$

satisfazendo $\alpha\left(s_{n}\right)=0$, para todo $n \in \mathbb{N}$. Pelo Lema 2.10, para cada $n \in \mathbb{N}$ existe um $s_{n}^{*}$, tal que

$$
s_{n} \leq s_{n}^{*} \leq s_{n+1} \text { e } \tau\left(s_{n}^{*}\right)=\alpha^{\prime}\left(s_{n}^{*}\right)=0 .
$$

Assim, temos as sequências crescentes $\left(s_{n}\right)$ e $\left(s_{n}^{*}\right)$ definidas no compacto $\left[s_{-}, s_{+}\right]$. Desta forma, existe $s_{0} \in\left[s_{-}, s_{+}\right]$, tal que

$$
\lim _{n \rightarrow+\infty} s_{n}=\lim _{n \rightarrow+\infty} s_{n}^{*}=s_{0} .
$$

Pela continuidade de $\alpha$ e $\tau$, temos

$$
\alpha\left(s_{0}\right)=\lim _{n \rightarrow \infty} \alpha\left(s_{n}\right)=0 \text { e } \tau\left(s_{0}\right)=\lim _{n \rightarrow \infty} \tau\left(s_{n}^{*}\right)=0,
$$

isso implica que $\nu\left(s_{0}\right)=1$, o que é uma contradição. Portanto, $\alpha$ zera no máximo em um número finito de pontos de $\mathbb{R}$. Além disso, pelos Lema 2.14 e 2.15, a função altura $\alpha$ converge para zero de forma estritamente monótona. Desta maneira, cada fim de $X$ converge para $\Gamma$ sem se auto-intersectar quando $t \rightarrow \pm \infty$.

Quando $0<a<2$, o sóliton $X$ forma uma sequência de laços que intersectam $\Gamma$ infinitas vezes e a altura máxima de cada laço é crescente enquanto $\nu$ é positivo e decrescente quando $\nu$ é negativo. Além disso, os fins de $X$ assintotam $\Gamma$, como podemos ver nas Figuras 2.3 e 2.4. Quando $a>2$, o sóliton intersecta $\Gamma$ um número finito de vezes e cada um se seus fins assintota $\Gamma$ sem se auto intersectar.

Proposição 2.18 Uma curva da esfera $X: \mathbb{R} \rightarrow \mathbb{S}^{2} \subset \mathbb{R}^{3}$ parametrizada pelo comprimento de arco

$$
X(s)=(\sin (\phi(s)) \cos (\theta(s)), \sin (\phi(s)) \sin (\theta(s)), \cos (\phi(s))),
$$


é um sóliton do FRC da esfera satisfazendo (2.5) se, e somente se, as funções $\theta$ e $\phi$ satisfazem

$$
\left\{\begin{array}{l}
\phi^{\prime \prime}=\theta^{\prime} \sin (\phi)\left(\theta^{\prime} \cos (\phi)-a \phi^{\prime} \sin (\phi)\right) \\
\theta^{\prime \prime}=\phi^{\prime}\left(a \phi^{\prime}-2 \theta^{\prime} \cot (\phi)\right) .
\end{array}\right.
$$

Prova. Seja $X$ uma curva de $\mathbb{S}^{2}$ parametrizada por (2.11). Como $X$ está parametrizada pelo comprimento de arco, o campo tangente unitário de $X$ é dado por

$$
T=\left(\phi^{\prime} \cos (\phi) \cos (\theta)-\theta^{\prime} \sin (\phi) \sin (\theta), \phi^{\prime} \cos (\phi) \sin (\theta)+\theta^{\prime} \sin (\phi) \cos (\theta),-\phi^{\prime} \sin (\phi)\right),
$$

onde

$$
\left(\phi^{\prime}\right)^{2}+\left(\theta^{\prime}\right)^{2} \sin ^{2}(\phi)=1
$$

e o campo normal unitário por

$$
N=\left(\phi^{\prime} \sin (\theta)+\theta^{\prime} \cos (\phi) \sin (\phi) \cos (\theta),-\phi^{\prime} \cos (\theta)+\theta^{\prime} \cos (\phi) \sin (\phi) \sin (\theta),-\theta^{\prime} \sin ^{2}(\phi)\right) .
$$

Segue de (1.3), que a curvatura de $X$ é dada por $\kappa=\left\langle T^{\prime}, N\right\rangle$, isto é,

$$
\kappa=\phi^{\prime \prime} \theta^{\prime} \sin (\phi)-\phi^{\prime} \theta^{\prime \prime} \sin (\phi)-\left(\theta^{\prime}\right)^{3} \cos (\phi) \sin (\phi)-2\left(\phi^{\prime}\right)^{2} \theta^{\prime} \cos (\phi) .
$$

Derivando (2.13), obtemos

$$
\phi^{\prime \prime} \phi^{\prime}+\theta^{\prime \prime} \theta^{\prime} \sin ^{2}(\phi)+\left(\theta^{\prime}\right)^{2} \phi^{\prime} \sin (\phi) \cos (\phi)=0 .
$$

Assim, $X$ satisfaz (2.5) se, e somente se,

$$
\phi^{\prime \prime} \theta^{\prime} \sin (\phi)-\phi^{\prime} \theta^{\prime \prime} \sin (\phi)-\left(\phi^{\prime}\right)^{2} \theta^{\prime} \cos (\phi)-\theta^{\prime} \cos (\phi)+a \phi^{\prime} \sin (\phi)=0 .
$$

Isolando $\phi^{\prime \prime}$ e $\theta^{\prime \prime}$ em (2.15) e (2.14), temos a equivalência com (2.12).

\subsection{Exemplos de Sólitons do FRC na Esfera}

Nesta secção, vamos usar a Proposição 2.18, para esboçar o traço de alguns sólitons do fluxo redutor de curvas na esfera. Como vimos na seção anterior, o formato das curvas 
depende da velocidade de rotação $a$. Nas figuras abaixo, vamos considerar as velocidades $a=0,5,1,2$ e 3 , respectivamente.

Nas Figuras 2.3 e 2.4 estamos visualizando sólitons do FRC $X(s), s \in \mathbb{R}$, na esfera quando $a=0,5$ e 1 . Os fins de $X$ convergem para $\Gamma$ quando $t \rightarrow \pm \infty$. Além disso, $X$ forma uma sequência de laços que intersecta $\Gamma$ infinitas vezes. Esta sequência de laços é crescente até um laço de altura máxima e depois decrescente assintotando $\Gamma$.

Nas Figuras 2.5 e 2.6 estamos visualizando sólitons do FRC $X(s), s \in \mathbb{R}$, na esfera quando $a=2$ e 3 , respectivamente. No Figura 2.5, podemos ver que $X$ não intersecta $\Gamma$ e cada um dos fins converge para $\Gamma$ sem se auto intersectar. Na Figura 2.5, podemos ver que, $X$ intersecta $\Gamma$ em um único ponto. Além disso, cada um dos fins converge para $\Gamma$ sem se auto-intersectar.

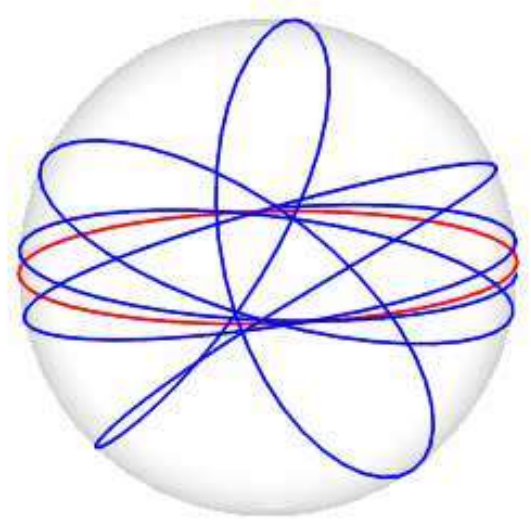

Figura 2.3: Sólitons do FRC na Esfera, $a=0,5$ 


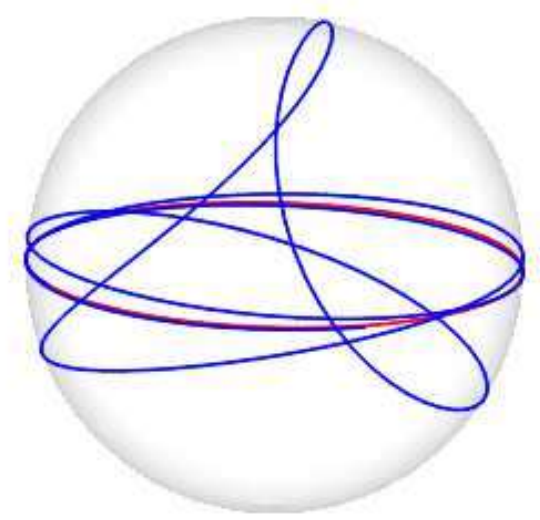

Figura 2.4: Sólitons do FRC na Esfera, $a=1$

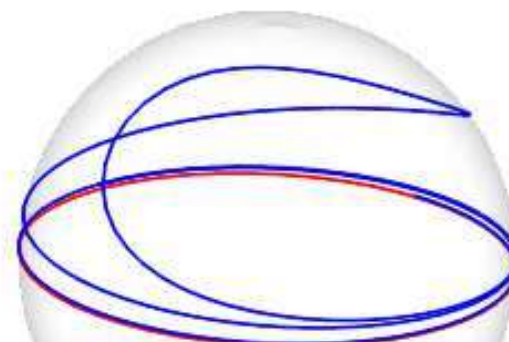

Figura 2.5: Sólitons do FRC na Esfera, $a=2$ 


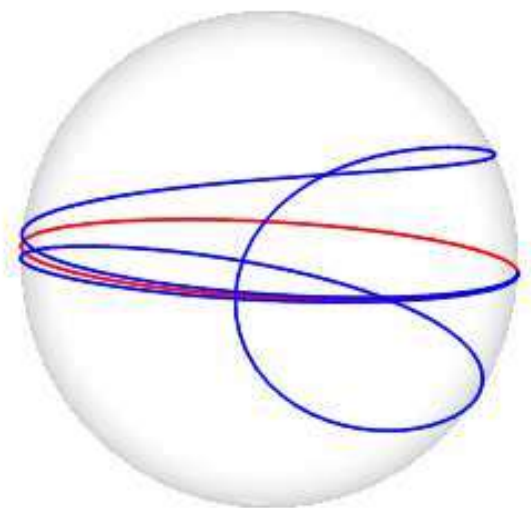

Figura 2.6: Sólitons do FRC na Esfera, a=3 


\section{CAPÍTULO 3}

\section{Soluções do FCM em Formas Espaciais por Hipersuperfícies}

Paralelas

O fluxo de curvatura média (FCM) é um fluxo do tipo gradiente para o funcional área [28]. Ele é uma equação diferencial parcial de evolução não linear para hipersuperfícies similar à equação do calor, com algumas diferenças importantes. O FCM se comporta como a equação do calor em um curto período de tempo.

Nos últimos anos, muitos estudos sobre soluções do fluxo de curvatura média tem sido apresentados. O tipo de solução mais estudado são as soluções autossimilares, isto é, soluções dadas pela composição de isometrias e homotetias. No espaço Euclidiano, os exemplos mais simples de solução autossimilar são as esferas e os cilindros, que são hipersuperfícies auto-contráteis (veja os Exemplos 1.20 e 1.21). Outros exemplos de hipersuperfícies auto-contráteis no espaço Euclidiano podem ser encontradas em [3, 7, 25] e hipersuperfícies de translação são encontradas em [8, 30, 36, 17].

Soluções autossimilares em outros espaços Riemannianos tem sido menos estudadas. Em [21], Hungerbühler e Smoczyk consideraram as soluções sólitons para o FCM, isto é, soluções evoluem pelo FCM por um grupo de isometrias do espaço ambiente. Vários exemplos de sólitons sobre variedades são discutidos neste trabalho. Outros tipos de 
soluções para o FCM também aparecem na literatura, como o trabalho de Liu e Terng [27], onde eles estudaram o FCM sobre subvariedades isoparamétricas do espaço Euclidiano e da esfera, e provaram que o fluxo preserva a condição de ser isoparamétrica e desenvolve singularidade em tempo finito.

Neste capítulo, vamos estudar as soluções do fluxo de curvatura média em formas espaciais por hipersuperfícies paralelas, isto é, as soluções do fluxo em que, a cada instante da deformação, a hipersuperfície obtida é paralela à hipersuperfície inicial.

Na Seção 3.1, vamos provar um teorema que caracteriza as hipersuperfície isoparamétricas como sendo as únicas hipersuperfícies de uma forma espacial que admitem uma solução do FCM por hipersuperfícies paralelas. Na seção 3.2, vamos obter explicitamente as soluções do fluxo de curvatura média por hipersuperfícies paralelas sobre as hipersuperfícies isoparamétricas do espaço Euclidiano. Na Seção 3.3, descrevemos a solução do fluxo de curvatura média por hipersuperfícies paralelas sobre as isoparamétricas do espaço hiperbólico. E para finalizar, na Seção 3.4, apresentamos a solução do fluxo de curvatura média por hipersuperfícies paralelas sobre algumas isoparamétricas da esfera.

\subsection{Teorema de Caracterização}

Nesta seção, vamos caracterizar a condição inicial de soluções do FCM em uma forma espacial por hipersuperfícies paralelas.

Vamos considerar as formas espaciais

$$
\mathbb{M}^{n+1}(\bar{\kappa})= \begin{cases}\mathbb{R}^{n+1} & \text { se } \bar{\kappa}=0 \\ \mathbb{S}^{n+1} \subset \mathbb{R}^{n+2} & \text { se } \bar{\kappa}=1 \\ \mathbb{H}^{n+1} \subset \mathbb{L}^{n+2} & \text { se } \bar{\kappa}=-1\end{cases}
$$

Definição 3.1 Seja $\widehat{F}: M^{n} \times I \rightarrow \mathbb{M}^{n+1}(\bar{\kappa})$ uma solução do fluxo de curvatura média em $\mathbb{M}^{n+1}(\bar{\kappa})$ com condição inicial $F: M^{n} \rightarrow \mathbb{M}^{n+1}(\bar{\kappa})$. Dizemos que $\widehat{F}$ é uma solução por hipersuperfícies paralelas se existe uma função $\xi: I \rightarrow \mathbb{R}$, tal que $\xi(0)=0$ e

$$
\widehat{F}^{t}(x)=c(\xi(t)) F(x)+s(\xi(t)) N(x)
$$

para todo $t \in I$, onde $c: \mathbb{R} \rightarrow \mathbb{R}$ e $s: \mathbb{R} \rightarrow \mathbb{R}$ são as funções definidas em (1.8). 
A seguir, vamos apresentar o principal teorema deste capitulo.

Teorema 3.2 Seja $F: M^{n} \rightarrow \mathbb{M}^{n+1}(\bar{\kappa})$ uma hipersuperfície da forma espacial $\mathbb{M}^{n+1}(\bar{\kappa})$. Então $F(M)$ é condição inicial de uma solução do fluxo de curvatura média por hipersuperfícies paralelas se, e somente se, $F(M)$ é isoparamétrica.

Prova. Suponha que $\widehat{F}^{t}$ dado por (3.1) é uma solução do FCM por hipersuperfícies paralelas. Derivando em relação a $t$, temos que

$$
\begin{aligned}
\frac{\partial}{\partial t} \widehat{F}(x, t) & =\xi^{\prime}(t)\left(c^{\prime}(\xi(t)) F(x)+s^{\prime}(\xi(t)) N(x)\right) \\
& =\xi^{\prime}(t)(-\bar{\kappa} s(\xi(t)) F(x)+c(\xi(t)) N(x))
\end{aligned}
$$

Substituindo em (1.13), temos

$$
\xi^{\prime}(t)(-\bar{\kappa} s(\xi(t)) F(x)+c(\xi(t)) N(x))=\widehat{H}^{t}(x) \widehat{N}^{t}(x)
$$

e pelo Lema 1.14, obtemos

$$
\xi^{\prime}(t)=\widehat{H}^{t}(x)
$$

Assim, pelo Lema 1.17, concluímos que $F(M)$ é isoparamétrica.

Reciprocamente, se $F(M)$ é isoparamétrica, seja $\xi(t)$ a única função que satisfaz a equação diferencial ordinária

$$
\xi^{\prime}(t)=\sum_{\imath=1}^{n} \frac{\bar{\kappa} s(\xi(t))+\kappa_{\imath} c(\xi(t))}{c(\xi(t))-\kappa_{\imath} s(\xi(t))}
$$

com $\xi(0)=0$, onde $\kappa_{\imath}$ é a $\imath$-ésima curvatura principal da hipersuperfície isoparamétrica $F(M)$. A existência de tal função é garantida pelo Teorema de Existência e Unicidade de EDO (veja [34]), pois a função

$$
\xi \mapsto \sum_{\imath=1}^{n} \frac{\bar{\kappa} s(\xi)+\kappa_{\imath} c(\xi)}{c(\xi)-\kappa_{\imath} s(\xi)},
$$

está definida e é diferenciável em uma vizinhança de 0 .

Defina

$$
\widehat{F}^{t}(x)=c(\xi(t)) F(x)+s(\xi(t)) N(x)
$$


Vamos mostra que $\widehat{F}^{t}(x)$ é solução do FCM. Derivando a equação, temos

$$
\begin{aligned}
\frac{\partial}{\partial t} \widehat{F}(x, t) & =\xi^{\prime}(t)\left(c^{\prime}(\xi(t)) F(x)+s^{\prime}(\xi(t)) N(x)\right) \\
& =\xi^{\prime}(t)(-\bar{\kappa} s(\xi(t)) F(x)+c(\xi(t)) N(x)) .
\end{aligned}
$$

Pelo Lema 1.14, temos que

$$
\widehat{N}^{t}(x)=-\bar{\kappa} s(\xi(t)) F(x)+c(\xi(t)) N(x)
$$

e pelo Lema 1.15

$$
\widehat{H}^{t}(x)=\sum_{\imath=1}^{n} \frac{\bar{\kappa} s(\xi(t))+\kappa_{\imath} c(\xi(t))}{c(\xi(t))-\kappa_{\imath} s(\xi(t))}=\xi^{\prime}(t) .
$$

Portanto,

$$
\frac{\partial}{\partial t} \widehat{F}^{t}(x)=\widehat{H}^{t}(x) \widehat{N}^{t}(x)
$$

e como $\xi(0)=0, \widehat{F}^{0}(x)=F(x)$.

Na demostração do teorema, podemos ver que encontrar solução do FCM sobre hipersuperfícies isoparamétricas de uma forma espacial é equivalente a integrar (3.2). Nas próximas seções, vamos integrar essa equação e explicitar as soluções do FCM sobre hipersuperfícies isoparamétricas.

\subsection{Soluções do FCM por Hipersuperfícies Paralelas em $\mathbb{R}^{n+1}$}

Nesta seção, vamos considerar as hipersuperfícies isoparamétricas do espaço Euclidiano e usar o Teorema 3.2 para construir soluções explícitas do fluxo de curvatura média por hipersuperfícies paralelas sobre tais hipersuperfícies. Observe que as soluções encontradas nessa seção são as mesmas apresentadas na Seção 1.4 vistas por outra perspectiva.

Pelo Teorema 1.9, as hipersuperfícies isoparamétricas $F: M^{n} \rightarrow \mathbb{R}^{n+1}$ são classificadas em: 
- Plano Euclidiano $\left(M^{n}=\mathbb{R}^{n}\right)$, quando todas as curvaturas principais de $F(M)$ são iguais a $\kappa=0$,

- Esfera $\left(M^{n}=\mathbb{S}^{n}\right)$, quando todas as curvaturas principais de $F(M)$ são iguais a $\kappa \neq 0$,

- Cilindro $\left(M^{n}=\mathbb{S}^{m_{1}} \times \mathbb{R}^{m_{2}}\right)$, quando temos $m_{1}$ curvaturas principais iguais a $\kappa \neq 0$ e as outras $m_{2}$ curvaturas principais iguais a zero.

Proposição 3.3 Seja $F: \mathbb{S}^{n} \rightarrow \mathbb{R}^{n+1}$ a imersão de uma esfera no espaço Euclidiano, com curvaturas principais iguais a $\kappa$. Então a solução do fluxo de curvatura média por hipersuperfícies paralelas com condição inicial $F(M)$ é

$$
\widehat{F}^{t}(x)=F(x)+\frac{1-\sqrt{1-2 n \kappa^{2} t}}{\kappa} N(x)
$$

para todo $t \in\left(-\infty, \frac{1}{2 n \kappa^{2}}\right)$. Além disso, a solução colapsa em um ponto quando $t \rightarrow \frac{1}{2 n \kappa^{2}}$.

Prova. Pelo Teorema 3.2, uma solução do fluxo de curvatura média sobre $F\left(\mathbb{S}^{n}\right)$ é dada por

$$
\widehat{F}^{t}(x)=F(x)+\xi(t) N(x)
$$

onde $\xi(t)$ satisfaz (3.2). Como as curvaturas principais são todas iguais a $\kappa$, esta equação é equivalente a

$$
\xi^{\prime}(t)=n \frac{\kappa}{1-\kappa \xi(t)} .
$$

Integrando a equação com condição inicial $\xi(0)=0$, temos

$$
\xi(t)=\frac{1-\sqrt{1-2 n \kappa^{2} t}}{\kappa}
$$

para todo $t \in\left(-\infty, \frac{1}{2 n \kappa^{2}}\right)$.

Além disso,

$$
\lim _{t \rightarrow \frac{1}{2 n \kappa^{2}}} \widehat{F}^{t}(x)=F(x)+\frac{1}{\kappa} N(x),
$$

diferenciando, temos

$$
\lim _{t \rightarrow \frac{1}{2 n \kappa^{2}}} d \widehat{F}_{x}^{t}=d F_{x}+\frac{1}{\kappa} d N_{x}=0
$$

pois $d F_{x}=-\frac{1}{\kappa} d N_{x}$. Portando, quando $t \rightarrow \frac{1}{2 n \kappa^{2}}$ a solução colapsa em um ponto. 
Exemplo 3.4 No intuito de visualizar a deformação, vamos considerar a esfera parametrizada

$$
F(\theta, \phi)=(\sin (\phi) \cos (\theta), \sin (\phi) \sin (\theta), \cos (\phi)),
$$

seu campo normal é

$$
N(\theta, \phi)=(-\sin (\phi) \cos (\theta),-\sin (\phi) \sin (\theta),-\cos (\phi)),
$$

e as curvaturas principais com respeito a $N$ são iguais a $\kappa=1$. Assim, pelo corolário anterior, a solução do FCM por paralelas sobre $F$ é

$$
\widehat{F}^{t}(\theta, \phi)=F(\theta, \phi)+(1-\sqrt{1-4 t}) N(\theta, \phi),
$$

isto é,

$$
\widehat{F}^{t}(\theta, \phi)=\sqrt{1-4 t} F(\theta, \phi)
$$

Podemos visualizar $\widehat{F}^{t}$ na Figura 3.1.
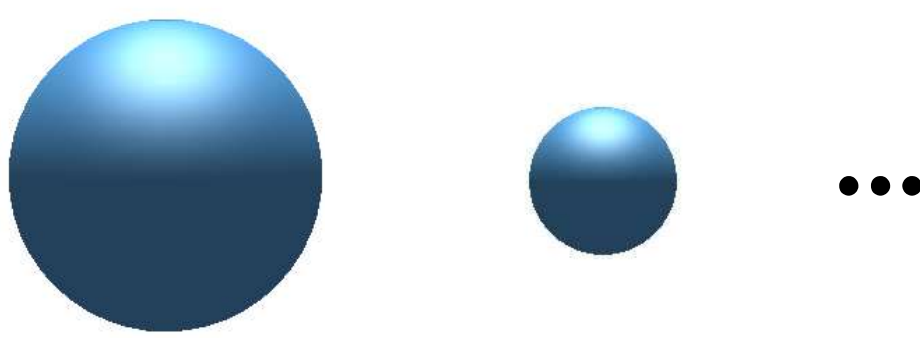

Figura 3.1: Fluxo de Curvatura Média sobre a Esfera Euclidiana

Proposição 3.5 Seja $F: \mathbb{S}^{m} \times \mathbb{R}^{n-m} \rightarrow \mathbb{R}^{n+1}$ a imersão de um cilindro no espaço Euclidiano, com $m$ curvaturas principais iguais a $\kappa \neq 0$ e $n-m$ curvaturas principais 
iguais a zero. Então a solução do fluxo de curvatura média por hipersuperfícies paralelas sobre $F(M)$ é

$$
\widehat{F}^{t}(x)=F(x)+\frac{1-\sqrt{1-2 m \kappa^{2} t}}{\kappa} N(x),
$$

para todo $t \in\left(-\infty, \frac{1}{2 m \kappa^{2}}\right)$. Além disso, a solução colapsa em uma hipersuperfície totalmente geodésica de dimensão $n-m$ no instante $t \rightarrow \frac{1}{2 m \kappa^{2}}$.

Prova. Pelo Teorema 3.2, a solução é dada por

$$
\widehat{F}^{t}(x)=F(x)+\xi(t) N(x)
$$

onde $\xi(t)$ satisfaz

$$
\xi^{\prime}(t)=m \frac{\kappa}{1-\kappa \xi(t)} .
$$

Integrando esta equação, obtemos

$$
\widehat{F}^{t}(x)=F(x)+\frac{1-\sqrt{1-2 m \kappa^{2} t}}{\kappa} N(x),
$$

para todo $t \in\left(\infty, 1 / 2 m \kappa^{2}\right)$.

Vamos mostrar que a solução colapsa em um plano totalmente geodésico de dimensão $n-m$. Quando $t \rightarrow \frac{1}{2 m \kappa^{2}}$, temos

$$
\lim _{t \rightarrow \frac{1}{2 m \kappa^{2}}} \widehat{F}^{t}(x)=F(x)+\frac{1}{\kappa} N(x),
$$

e pelo Lema 1.14

$$
\widehat{N}^{t}(x)=N(x) .
$$

Tomando a diferencial, temos

$$
\lim _{t \rightarrow \frac{1}{2 m \kappa^{2}}} d \widehat{F}_{x}^{t}=d F_{x}+\frac{1}{\kappa} d N_{x}
$$

Assim, se $V_{1} \in T \mathbb{S}^{m}$, temos

$$
\lim _{t \rightarrow \frac{1}{2 m \kappa^{2}}} d \widehat{F}_{x}^{t}\left(V_{1}\right)=d F_{x}\left(V_{1}\right)+\frac{1}{\kappa} d N_{x}\left(V_{1}\right)=0,
$$

e se $V_{2} \in T \mathbb{R}^{n-m}$

$$
\lim _{t \rightarrow \frac{1}{2 m \kappa^{2}}} d \widehat{F}_{x}^{t}\left(V_{2}\right)=d F_{x}\left(V_{2}\right)+\frac{1}{\kappa} d N_{x}\left(V_{2}\right)=d F_{x}\left(V_{2}\right) .
$$


Assim, quando $t \rightarrow \frac{1}{2 m \kappa^{2}}, \widehat{F}^{t}$ converge para uma subvariedade de dimensão $n-m$. Além disso,

$$
\lim _{t \rightarrow \frac{1}{2 m \kappa^{2}}} d N_{x}^{t}\left(V_{2}\right)=d N_{x}\left(V_{2}\right)=0
$$

Portanto, quando $t \rightarrow \frac{1}{2 m \kappa^{2}}, \widehat{F}^{t}(M)$ converge para um plano de dimensão $n-m$.

Exemplo 3.6 Considere o cilindro parametrizada

$$
F(\theta, \phi)=(\cos (\theta), \sin (\theta), \phi),
$$

seu campo normal é

$$
N(\theta, \phi)=(-\cos (\theta),-\sin (\theta), 0),
$$

e as curvaturas principais com respeito a $N$ são $\kappa_{1}=1$ e $\kappa_{2}=0$. Assim a solução do fluxo de curvatura média sobre $F$ é

$$
\widehat{F}^{t}(\theta, \phi)=(\sqrt{1-2 t} \cos (\theta), \sqrt{1-2 t} \sin (\theta), \phi),
$$

que pode ser visualizado na Figura 3.2.
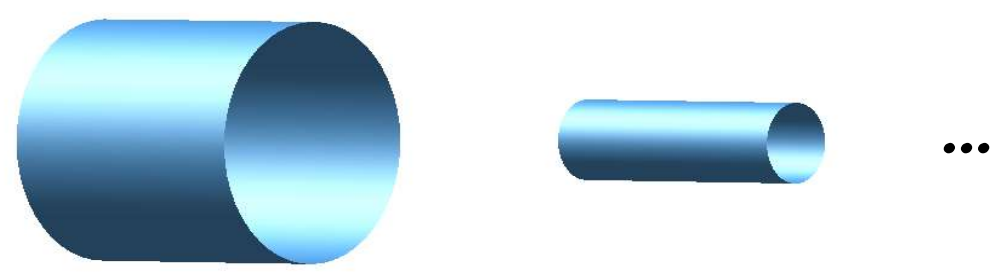

Figura 3.2: Fluxo de Curvatura Média sobre a Cilindro Euclidiano 


\subsection{Soluções do FCM por Hipersuperfícies Paralelas em $\mathbb{H}^{n+1}$}

Nesta seção, vamos considerar as hipersuperfícies isoparamétricas do espaço hiperbólico e usar o Teorema 3.2 para construir soluções explícitas do fluxo de curvatura média por hipersuperfícies paralelas sobre tais hipersuperfícies.

Pelo Teorema 1.10, temos as hipersuperfícies totalmente umbílicas, com todas as curvaturas principais iguais a $\kappa$, que são classificadas como:

- $M^{n}=\mathbb{H}^{n}$, se $0 \leq|\kappa|<1$,

- $M^{n}=\mathbb{R}^{n}$, se $\kappa= \pm 1$,

- $M^{n}=\mathbb{S}^{n}$, se $|\kappa|>1$

e as não umbílicas, com duas curvaturas principais distintas $\kappa_{1}$ e $\kappa_{2}$, que são classificadas como:

- $M^{n}=\mathbb{S}^{m_{1}} \times \mathbb{H}^{m_{2}}$, onde $\kappa_{1} \kappa_{2}=1$.

Proposição 3.7 Seja $F: \mathbb{R}^{n} \rightarrow \mathbb{H}^{n+1} \subset \mathbb{L}^{n+2}$ a imersão de uma horoesfera no espaço hiperbólico, com campo normal unitário $N$ e curvaturas principais iguais a $\kappa= \pm 1$. Então a solução do fluxo de curvatura média por hipersuperfícies paralelas sobre $F(M)$ é

$$
\widehat{F}^{t}(x)=\cosh (n t) F(x)+\kappa \sinh (n t) N(x),
$$

para todo $t \in \mathbb{R}$. Além disso, $\widehat{F}^{t}\left(\mathbb{R}^{n}\right)$ é uma horoesfera para cada $t \in \mathbb{R}$.

Prova. Pelo Teorema 3.2 a solução é dada por

$$
\widehat{F}^{t}(x)=\cosh (\xi(t)) F(x)+\sinh (\xi(t)) N(x),
$$

onde $\xi$ satisfaz (3.2). Como $\kappa= \pm 1, \xi(t)$ satisfaz

$$
\xi^{\prime}(t)=-n \frac{\sinh (\xi(t))-\kappa \cosh (\xi(t))}{\cosh (\xi(t))-\kappa \sinh (\xi(t))}=\kappa n .
$$


Integrando esta equação, como $\xi(0)=0$, temos que

$$
\xi(t)=\kappa n t
$$

para todo $t \in \mathbb{R}$. Assim, segue (3.3).

Agora vamos mostrar que $\widehat{F}^{t}\left(\mathbb{R}^{n}\right)$ é uma horoesfera para todo $t \in \mathbb{R}$. Seja $\left\{E_{1}, \ldots, E_{n}\right\}$ uma base de autovalores de $h$ em $T \mathbb{R}^{n}$. Assim, do Lema 1.15, temos que

$$
\widehat{\kappa}_{\imath}^{t}=-\frac{\sinh (n t)-\kappa \cosh (n t)}{\cosh (n t)-\kappa \sinh (n t)}=\kappa .
$$

Portanto, segue o resultado.

Exemplo 3.8 Considere a horoesfera em $\mathbb{H}^{3}$ parametrizada por

$$
F(\theta, \phi)=\left(\frac{3+2 \sin \phi}{2(1+\sin \phi)}, \frac{\cos \phi \cos \theta}{1+\sin \phi}, \frac{\cos \phi \sin \theta}{1+\sin \phi}, \frac{1+2 \sin \phi}{2(1+\sin \phi)}\right)
$$

seu campo normal é

$$
N(\theta, \phi)=\left(\frac{2+\sin \phi}{2(1+\sin \phi)}, \frac{\cos \phi \cos \theta}{1+\sin \phi}, \frac{\cos \phi \sin \theta}{1+\sin \phi}, \frac{2+3 \sin \phi}{2(1+\sin \phi)}\right),
$$

e as curvaturas principais com respeito a $N$ são todas iguais a $\kappa=1$. Assim, a solução (3.3) do FCM por paralelas sobre $F$ pode ser visualizado, via projeção de $\mathbb{H}^{3}$ no modelo do semi-espaço, na Figura 3.3.
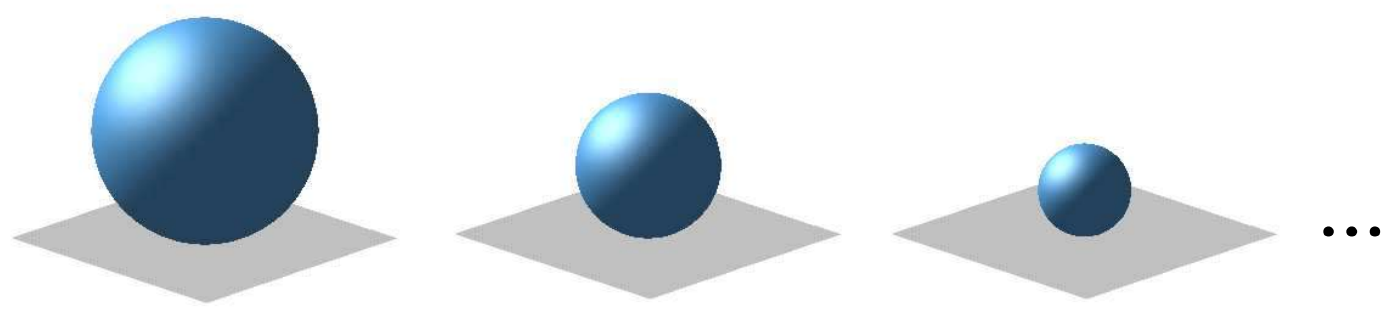

Figura 3.3: Fluxo de Curvatura Média sobre a Horoesfera 
Proposição 3.9 Seja $F: M^{n} \rightarrow \mathbb{H}^{n+1} \subset \mathbb{L}^{n+2}$ uma imersão de uma hipersuperfície totalmente umbílica no espaço hiperbólico, com campo normal $N$ e curvaturas principais não nulas iguais a $\kappa \neq \pm 1$. Então a solução do fluxo de curvatura média por hipersuperfícies paralelas sobre $F(M)$ é

$$
\widehat{F}^{t}(x)=\frac{\kappa^{2} e^{-n t}-\sqrt{1-\kappa^{2}+\kappa^{2} e^{-2 n t}}}{\kappa^{2}-1} F(x)+\frac{\kappa e^{-n t}-\kappa \sqrt{1-\kappa^{2}+\kappa^{2} e^{-2 n t}}}{\kappa^{2}-1} N(x) .
$$

Além disso,

1. Se $0<|\kappa|<1$ a solução está definida para todo $t \in \mathbb{R}$ e converge para um espaço hiperbólico totalmente geodésico quando $t \rightarrow+\infty$.

2. Se $|\kappa|>1$ a solução está definida para $t \in\left(-\infty, \frac{1}{2 n} \ln \left(\frac{\kappa^{2}}{\kappa^{2}-1}\right)\right)$ e colapsa em um ponto quando $t \rightarrow \frac{1}{2 n} \ln \left(\frac{\kappa^{2}}{\kappa^{2}-1}\right)$.

Prova. Pelo Teorema 3.2, a solução é dada por

$$
\widehat{F}^{t}(x)=\cosh (\xi(t)) F(x)+\sinh (\xi(t)) N(x),
$$

onde $\xi(t)$ satisfaz

$$
\xi^{\prime}(t)=-n \frac{\sinh (\xi(t))-\kappa \cosh (\xi(t))}{\cosh (\xi(t))-\kappa \sinh (\xi(t))}
$$

Assim

$$
\frac{d}{d t} \ln |\sinh (\xi(t))-\kappa \cosh (\xi(t))|=-n,
$$

integrando esta equação, como $\xi(0)=0$, temos

$$
\sinh (\xi(t))-\kappa \cosh (\xi(t))=-\kappa e^{-n t}
$$

Isolando $\sinh (\xi(t))$, elevando ao quadrado e organizando os termos, temos

$$
\left(\kappa^{2}-1\right) \cosh ^{2}(\xi(t))-2 \kappa^{2} e^{-n t} \cosh (\xi(t))+\kappa^{2} e^{-2 n t}+1=0,
$$

resolvendo a equação, obtemos

$\cosh (\xi(t))=\frac{\kappa^{2} e^{-n t}-\sqrt{1-\kappa^{2}+\kappa^{2} e^{-2 n t}}}{\kappa^{2}-1}$ e $\sinh (\xi(t))=\frac{\kappa e^{-n t}-\kappa \sqrt{1-\kappa^{2}+\kappa^{2} e^{-2 n t}}}{\kappa^{2}-1}$.

Portanto, segue (3.4) e a solução está definido para todo $t \in \mathbb{R}$ se $0<|\kappa|<1$, e se $|\kappa|>1$ cla está definida para $t \in\left(-\infty, \frac{1}{2 n} \ln \left(\frac{\kappa^{2}}{\kappa^{2}-1}\right)\right)$. 
Para o item 1 , considere que $0<|\kappa|<1$. Seja $\left\{E_{1}, \ldots, E_{n}\right\}$ uma base ortonormal de autovalores da segunda forma fundamental de $F\left(\mathbb{H}^{n}\right)$. Pelo Lema 1.15 e (3.4), a primeira forma fundamental de $\widehat{F}^{t}\left(\mathbb{H}^{n}\right)$ em relação a esta base é

$$
\widehat{g}^{t}\left(E_{\imath}, E_{\jmath}\right)=\left(1-\kappa^{2}+\kappa^{2} e^{-2 n t}\right) \delta_{\imath \jmath}
$$

e as curvaturas principais são todas iguais a

$$
\widehat{\kappa}^{t}=\frac{\kappa}{\sqrt{\kappa^{2}+\left(1-\kappa^{2}\right) e^{2 n t}}}
$$

Considerando $t \rightarrow+\infty$, temos

$$
\lim _{t \rightarrow+\infty} \widehat{g}^{t}\left(E_{\imath}, E_{\jmath}\right)=\left(1-\kappa^{2}\right) \delta_{\imath \jmath}
$$

$\mathrm{e}$

$$
\lim _{t \rightarrow+\infty} \widehat{\kappa}^{t}=0
$$

Assim a solução assintota uma hipersuperfície totalmente geodésica quando $t \rightarrow+\infty$.

Para o item 2, considere $|\kappa|>1$. Seja $\left\{E_{1}, \ldots, E_{n}\right\}$ uma base ortonormal de autovalores da segunda forma fundamental de $F\left(\mathbb{S}^{n}\right)$. Pelo Lema 1.15 e (3.4), a primeira forma fundamental de $\widehat{F}^{t}\left(\mathbb{S}^{n}\right)$ em relação a esta base é

$$
\widehat{g}^{t}\left(E_{\imath}, E_{\jmath}\right)=\left(1-\kappa^{2}+\kappa^{2} e^{-2 n t}\right) \delta_{\imath \jmath}
$$

fazendo $t^{*}=\frac{1}{2 n} \ln \left(\frac{\kappa^{2}}{\kappa^{2}-1}\right)$, temos

$$
\lim _{t \rightarrow t^{*}} \widehat{g}^{t}\left(E_{\imath}, E_{\jmath}\right)=0
$$

Portando a solução colapsa em um ponto.

Exemplo 3.10 Considere a imersão $F: \mathbb{H}^{2} \rightarrow \mathbb{H}^{3} \subset \mathbb{L}^{4}$ dada por por

$$
F(\theta, \phi)=\left(\frac{3+2 \cos \phi}{1+2 \cos \phi}, \frac{2 \cos \phi \cos \theta}{1+2 \cos \phi}, \frac{2 \cos \phi \sin \theta}{1+2 \cos \phi}, \frac{2+2 \cos \phi}{1+2 \cos \phi}\right),
$$

seu campo normal é

$$
N(\theta, \phi)=\left(2 \frac{1+\cos \phi}{1+2 \cos \phi}, \frac{\sin \phi \cos \theta}{1+2 \cos \phi}, \frac{\sin \phi \sin \theta}{1+2 \cos \phi}, \frac{2+3 \cos \phi}{1+2 \cos \phi}\right),
$$



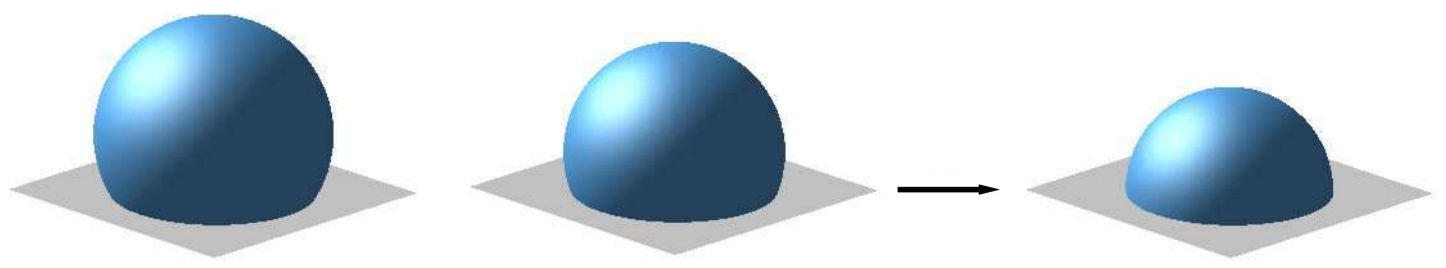

Figura 3.4: Fluxo de Curvatura Média sobre a Paralela $(0<|\kappa|<1)$

e as curvaturas principais com respeito a $N$ são todas iguais a $\kappa=-\frac{1}{2}$. Assim, a solução do fluxo de curvatura média sobre $F$ é dada por

$$
\widehat{F}^{t}(x)=\frac{-e^{-2 t}+2 \sqrt{e^{-4 t}+3}}{3} F(x)+\frac{2 e^{-2 t}-2 \sqrt{e^{-4 t}+3}}{3} N(x),
$$

e pode ser visualizado, via projeção no modelo do semi-espaço, na Figura 3.4.

Exemplo 3.11 Considere a esfera geodésica de $\mathbb{H}^{3}$ parametrizada por

$$
F(\theta, \phi)=\left(\frac{3+2 \cos \phi}{2+\cos \phi}, \frac{\cos \phi \cos \theta}{2+\cos \phi}, \frac{2 \cos \phi \sin \theta}{2+\cos \phi}, \frac{2+2 \cos \phi}{2+\cos \phi}\right)
$$

seu campo normal é

$$
N(\theta, \phi)=\left(2 \frac{1+\cos \phi}{2+\cos \phi}, 2 \frac{\sin \phi \cos \theta}{2+\cos \phi}, 2 \frac{\sin \phi \sin \theta}{2+\cos \phi}, \frac{2+3 \cos \phi}{2+\cos \phi}\right)
$$

e as curvaturas principais com respeito a $N$ são todas iguais a $\kappa=-2$. Assim, a solução do fluxo de curvatura média sobre $F$ é dada por

$$
\widehat{F}^{t}(x)=\frac{4 e^{-2 t}-\sqrt{4 e^{-4 t}-3}}{3} F(x)+\frac{-2 e^{-2 t}+2 \sqrt{4 e^{-4 t}-3}}{3} N(x),
$$

e pode ser visualizado, via projeção no modelo do semi-espaço, na Figura 3.5.

Proposição 3.12 Seja $F: \mathbb{S}^{m_{1}} \times \mathbb{H}^{m_{2}} \rightarrow \mathbb{H}^{n+1} \subset \mathbb{L}^{n+2}$ a imersão de um cilindro no espaço hiperbólico, com $m_{1}$ curvaturas principais iguais a $\kappa_{1}$ e $m_{2}$ curvaturas principais 

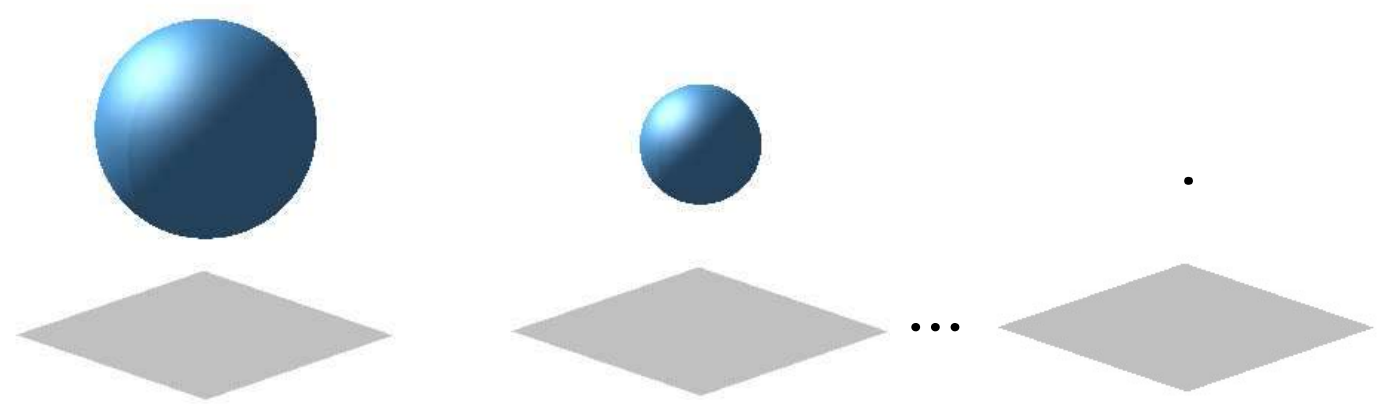

Figura 3.5: Fluxo de Curvatura Média sobre a Esfera de Geodésica de $\mathbb{H}^{3}(|\kappa|>1)$

iguais a $\kappa_{2}$, tais que $\kappa_{1} \kappa_{2}=1$. Então a solução do fluxo de curvatura média por hipersuperfícies paralelas sobre $F\left(\mathbb{S}^{m_{1}} \times \mathbb{H}^{m_{2}}\right)$ é

$$
\widehat{F}^{t}(x)=\cosh (\xi(t)) F(x)+\sinh (\xi(t)) N(x),
$$

onde

$$
\cosh (\xi(t))=\frac{1}{2} \sqrt{\frac{\ell(t)-\sqrt{\ell^{2}(t)-\mu^{2}+4}}{\mu-2}}+\frac{1}{2} \sqrt{\frac{\ell(t)+\sqrt{\ell^{2}(t)-\mu^{2}+4}}{\mu+2}}
$$

$e$

$$
\sinh (\xi(t))=\frac{1}{2} \sqrt{\frac{\ell(t)-\sqrt{\ell^{2}(t)-\mu^{2}+4}}{\mu-2}}-\frac{1}{2} \sqrt{\frac{\ell(t)+\sqrt{\ell^{2}(t)-\mu^{2}+4}}{\mu+2}} .
$$

para todo $t \in\left(-\infty, \frac{1}{2 n} \ln \left(\frac{m_{1} \kappa_{1}+m_{2} \kappa_{2}}{m_{1}\left(\kappa_{1}-\kappa_{2}\right)}\right)\right)$, com

$$
\ell(t)=(\mu-\omega) e^{-2 n t}+\omega, \quad \mu=\kappa_{1}+\kappa_{2} \quad e \quad \omega=-\frac{m_{1}-m_{2}}{n}\left(\kappa_{1}-\kappa_{2}\right) .
$$

Além disso, esta solução colapsa em uma variedade totalmente geodésica de $\mathbb{H}^{n+1}$ de dimensão $m_{2}$ quando $t \rightarrow \frac{1}{2 n} \ln \left(\frac{m_{1} \kappa_{1}+m_{2} \kappa_{2}}{m_{1}\left(\kappa_{1}-\kappa_{2}\right)}\right)$.

Prova. Pelo Corolário 3.2 a solução é dada por

$$
\widehat{F}^{t}(x)=\cosh (\xi(t)) F(x)+\sinh (\xi(t)) N(x),
$$


onde $\xi(t)$ satisfaz (3.2), isto é,

$$
\xi^{\prime}(t)=-m_{1} \frac{\sinh (\xi(t))-\kappa_{1} \cosh (\xi(t))}{\cosh (\xi(t))-\kappa_{1} \sinh (\xi(t))}-m_{2} \frac{\sinh (\xi(t))-\kappa_{2} \cosh (\xi(t))}{\cosh (\xi(t))-\kappa_{2} \sinh (\xi(t))} .
$$

Reduzindo ao mesmo denominador, reagrupando os termos, segue do fato que $m_{1}+m_{2}=n$

$$
\xi^{\prime}(t)=-n \frac{2 \sinh (2 \xi(t))-\left(\kappa_{1}+\kappa_{2}\right) \cosh (2 \xi(t))-\frac{m_{1}-m_{2}}{n}\left(\kappa_{1}-\kappa_{2}\right)}{2 \cosh (2 \xi(t))-\left(\kappa_{1}+\kappa_{2}\right) \sinh (2 \xi(t))} .
$$

Desta forma

$$
\frac{d}{d t} \ln |2 \sinh (2 \xi(t))-\mu \cosh (2 \xi(t))+\omega|=-2 n .
$$

Integrando os termos e usando que $\xi(0)=0$, com a notação (3.5), temos

$$
2 \sinh (2 \xi(t))-\mu \cosh (2 \xi(t))+\omega=(\omega-\mu) e^{-2 n t}
$$

Elevando ao quadrado, usando (3.5) e expressando esta equação em termos de $\cosh (2 \xi(t))$, temos

$$
\left(\mu^{2}-4\right) \cosh ^{2}(2 \xi(t))-2 \mu \ell(t) \cosh (2 \xi(t))+\ell^{2}(t)+4=0 .
$$

Assim

$$
\cosh (2 \xi(t))=\frac{\mu \ell(t)-2 \sqrt{\ell^{2}(t)-\mu^{2}+4}}{\mu^{2}-4} \text { e } \sinh (2 \xi(t))=\frac{2 \ell(t)-\mu \sqrt{\ell^{2}(t)-\mu^{2}+4}}{\mu^{2}-4} .
$$

Portando, colocando em termos de $\cosh (\xi(t))$ e $\sinh (\xi(t))$, temos a primeira parte do corolário.

Além disso, a solução está definida para todo $t$, tal que

$$
\ell^{2}(t)-\mu^{2}+4 \geq 0
$$

assim,como $-\mu^{2}+4=-\left(\kappa_{1}-\kappa_{2}\right)^{2}$ e $\omega-\mu=-\frac{2}{n}\left(m_{1} \kappa_{1}+m_{2} \kappa_{2}\right)$, temos que

$$
t \leq \frac{1}{2 n} \ln \left(\frac{\sqrt{\mu^{2}-4}-\omega}{\mu-\omega}\right)=\frac{1}{2 n} \ln \left(\frac{m_{1} \kappa_{1}+m_{2} \kappa_{2}}{m_{1}\left(\kappa_{1}-\kappa_{2}\right)}\right) \text {. }
$$

Agora, vamos mostrar que a solução colapsa em uma variedade totalmente geodésica em $t^{*}=\frac{1}{2 n} \ln \left(\frac{m_{1} \kappa_{1}+m_{2} \kappa_{2}}{m_{1}\left(\kappa_{1}-\kappa_{2}\right)}\right)$. Sem perda de generalidade, podemos considerar $0<\kappa_{2}<\kappa_{1}$. Fazendo $t \rightarrow t^{*}$

$$
\lim _{t \rightarrow t^{*}} \cosh (\xi(t))=\frac{1}{2} \sqrt{\frac{\kappa_{1}-\kappa_{2}}{\kappa_{1}+\kappa_{2}-2}}+\frac{1}{2} \sqrt{\frac{\kappa_{1}-\kappa_{2}}{\kappa_{1}+\kappa_{2}+2}}=\frac{1}{2} \frac{\sqrt{\kappa_{1}+\kappa_{2}+2}+\sqrt{\kappa_{1}+\kappa_{2}-2}}{\sqrt{\kappa_{1}-\kappa_{2}}},
$$


$\mathrm{e}$

$\lim _{t \rightarrow t^{*}} \sinh (\xi(t))=\frac{1}{2} \sqrt{\frac{\kappa_{1}-\kappa_{2}}{\kappa_{1}+\kappa_{2}-2}}-\frac{1}{2} \sqrt{\frac{\kappa_{1}-\kappa_{2}}{\kappa_{1}+\kappa_{2}+2}}=\frac{1}{2} \frac{\sqrt{\kappa_{1}+\kappa_{2}+2}-\sqrt{\kappa_{1}+\kappa_{2}-2}}{\sqrt{\kappa_{1}-\kappa_{2}}}$.

Seja $\left\{E_{1}, \ldots, E_{m_{1}}, E_{m_{1}+1}, \ldots, E_{m_{1}+m_{2}}=E_{n}\right\}$ uma base ortonormal de autovalores de $h$ em $T M$, onde $E_{1}, \ldots, E_{m_{1}}$ são tangentes a $\mathbb{S}^{m_{1}}$ e $E_{m_{1}+1}, \ldots, E_{n}$ são tangentes a $\mathbb{H}^{m_{2}}$.

Para $a, b=1, \ldots, m_{1}, \imath, \jmath=m_{m_{1}+1}, \ldots, n$, usando o Lema 1.15 , temos

$$
\begin{aligned}
\lim _{t \rightarrow t^{*}} \widehat{g}^{t}\left(E_{a}, E_{b}\right) & =\lim _{t \rightarrow t^{*}}\left(\cosh (\xi(t))-\kappa_{1} \sinh (\xi(t))\right)^{2} \delta_{a b} \\
& =\frac{\left[\sqrt{\kappa_{1}+\kappa_{2}+2}+\sqrt{\kappa_{1}+\kappa_{2}-2}-\kappa_{1}\left(\sqrt{\kappa_{1}+\kappa_{2}+2}-\sqrt{\kappa_{1}+\kappa_{2}-2}\right)\right]^{2}}{4\left(\kappa_{1}-\kappa_{2}\right)} \delta_{a b} \\
& =\frac{\left[\left(1-\kappa_{1}\right) \sqrt{\kappa_{1}+\kappa_{2}+2}+\left(1+\kappa_{1}\right) \sqrt{\kappa_{1}+\kappa_{2}-2}\right]^{2}}{4\left(\kappa_{1}-\kappa_{2}\right)} \delta_{a b} \\
& =\frac{\left(1-\kappa_{1}\right)^{2}\left(\kappa_{1}+\kappa_{2}+2\right)+\left(1+\kappa_{1}\right)^{2}\left(\kappa_{1}+\kappa_{2}-2\right)+2\left(1-\kappa_{1}^{2}\right)\left(\kappa_{1}-\kappa_{2}\right)}{4\left(\kappa_{1}-\kappa_{2}\right)} \delta_{a b} \\
& =0,
\end{aligned}
$$

$\lim _{t \rightarrow t^{*}} \widehat{g}^{t}\left(E_{\imath}, E_{\jmath}\right)=\lim _{t \rightarrow t^{*}}\left(\cosh (\xi(t))-\kappa_{2} \sinh (\xi(t))\right)^{2} \delta_{\imath \jmath}$

$$
\begin{aligned}
& =\frac{\left[\sqrt{\kappa_{1}+\kappa_{2}+2}+\sqrt{\kappa_{1}+\kappa_{2}-2}-\kappa_{2}\left(\sqrt{\kappa_{1}+\kappa_{2}+2}-\sqrt{\kappa_{1}+\kappa_{2}-2}\right)\right]^{2}}{4\left(\kappa_{1}-\kappa_{2}\right)} \delta_{\imath \jmath} \\
& =\frac{\left[\left(1-\kappa_{2}\right) \sqrt{\kappa_{1}+\kappa_{2}+2}+\left(1+\kappa_{2}\right) \sqrt{\kappa_{1}+\kappa_{2}-2}\right]^{2}}{4\left(\kappa_{1}-\kappa_{2}\right)} \delta_{\imath \jmath} \\
& =\frac{\left(1-\kappa_{2}\right)^{2}\left(\kappa_{1}+\kappa_{2}+2\right)+\left(1+\kappa_{2}\right)^{2}\left(\kappa_{1}+\kappa_{2}-2\right)+2\left(1-\kappa_{2}^{2}\right)\left(\kappa_{1}-\kappa_{2}\right)}{4\left(\kappa_{1}-\kappa_{2}\right)} \delta_{\imath \jmath} \\
& =\kappa_{2}\left(\kappa_{1}-\kappa_{2}\right) \delta_{\imath \jmath}
\end{aligned}
$$

e

$$
\lim _{t \rightarrow t^{*}} \widehat{g}^{t}\left(E_{a}, E_{\jmath}\right)=0 .
$$

Assim, a solução colapsa em uma subvariedade $m_{2}$ dimensional. Além disso,

$$
\begin{aligned}
\lim _{t \rightarrow t^{*}} \widehat{h}^{t}\left(E_{\imath}, E_{\jmath}\right) & =\lim _{t \rightarrow t^{*}}\left(\sinh (\xi(t))-\kappa_{2} \cosh (\xi(t))\right)^{2} \delta_{\imath \jmath} \\
& =\frac{\left[\sqrt{\kappa_{1}+\kappa_{2}+2}-\sqrt{\kappa_{1}+\kappa_{2}-2}-\kappa_{2}\left(\sqrt{\kappa_{1}+\kappa_{2}+2}+\sqrt{\kappa_{1}+\kappa_{2}-2}\right)\right]^{2}}{4\left(\kappa_{1}-\kappa_{2}\right)} \delta_{\imath \jmath} \\
& =\frac{\left[\left(1-\kappa_{2}\right) \sqrt{\kappa_{1}+\kappa_{2}+2}-\left(1+\kappa_{2}\right) \sqrt{\kappa_{1}+\kappa_{2}-2}\right]^{2}}{4\left(\kappa_{1}-\kappa_{2}\right)} \delta_{\imath \jmath} \\
& =\frac{\left(1-\kappa_{2}\right)^{2}\left(\kappa_{1}+\kappa_{2}+2\right)+\left(1+\kappa_{2}\right)^{2}\left(\kappa_{1}+\kappa_{2}-2\right)-2\left(1-\kappa_{2}^{2}\right)\left(\kappa_{1}-\kappa_{2}\right)}{4\left(\kappa_{1}-\kappa_{2}\right)} \delta_{\imath \jmath} \\
& =0,
\end{aligned}
$$


portanto, a variedade é totalmente geodésica.

Exemplo 3.13 Considere a cilindro hiperbólico em $\mathbb{H}^{3} \subset \mathbb{L}^{4}$ parametrizado por

$$
F(\theta, \phi)=(\sqrt{2} \cosh \phi, \cos \theta, \sin \theta, \sqrt{2} \sinh \phi)
$$

O campo unitário normal a $F$ é

$$
N(\theta, \phi)=(\cosh \phi, \sqrt{2} \cos \theta, \sqrt{2} \sin \theta, \sinh \phi),
$$

e as curvaturas principais com respeito a $N$ são $\kappa_{1}=\sqrt{2}$ e $\kappa_{2}=\frac{\sqrt{2}}{2}$.

Assim, a solução do fluxo de curvatura média sobre $F$ é dada por

$$
\widehat{F}^{t}(x)=\cosh (\xi(t)) F(x)+\sinh (\xi(t)) N(x),
$$

onde

$$
\cosh (\xi(t))=\frac{1}{2} \sqrt{\frac{3 e^{-4 t}-\sqrt{9 e^{-8 t}-1}}{3-2 \sqrt{2}}}+\frac{1}{2} \sqrt{\frac{3 e^{-4 t}+\sqrt{9 e^{-8 t}-1}}{3+2 \sqrt{2}}}
$$

$\mathrm{e}$

$$
\sinh (\xi(t))=\frac{1}{2} \sqrt{\frac{3 e^{-4 t}-\sqrt{9 e^{-8 t}-1}}{3-2 \sqrt{2}}}-\frac{1}{2} \sqrt{\frac{3 e^{-4 t}+\sqrt{9 e^{-8 t}-1}}{3+2 \sqrt{2}}},
$$

que pode ser visualizado, via projeção no modelo da bola de Poincaré do espaço hiperbólico, na Figura 3.6.
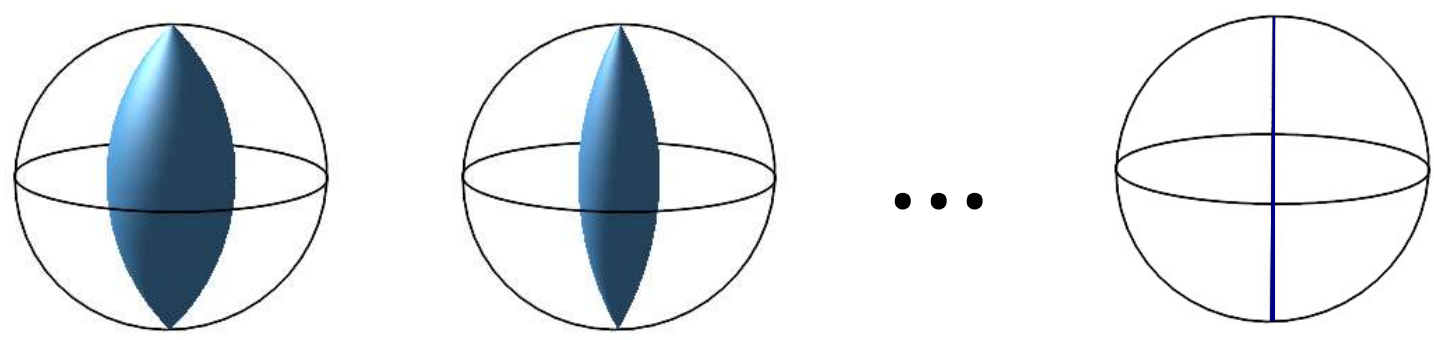

Figura 3.6: Fluxo de Curvatura Média sobre o Cilindro Hiperbólico 


\subsection{Soluções do FCM por Hipersuperfícies Paralelas em $\mathbb{S}^{n+1}$}

Nesta seção, vamos estudar a solução do FCM das hipersuperfícies umbílicas de $\mathbb{S}^{n+1}$ e do toro de Hopf da esfera $\mathbb{S}^{3}$. O fluxo de curvatura média sobre superfícies isoparamétricas na esfera foi estudado por Liu e Terng em [27]. Neste trabalho eles provaram que a solução colapsa em uma subvariedade focal.

Proposição 3.14 Seja $F: M^{n} \rightarrow \mathbb{S}^{n+1} \subset \mathbb{R}^{n+2}$ uma imersão de uma hipersuperfície totalmente umbílica na esfera $\mathbb{S}^{n+1}$, com campo normal unitário $N$ e curvaturas principais iguais a $\kappa \neq 0$. Então a solução do fluxo de curvatura média por hipersuperfícies paralelas sobre $F(M)$ é

$$
\widehat{F}^{t}(x)=\frac{\kappa^{2} e^{n t}+\sqrt{\kappa^{2}+1-\kappa^{2} e^{2 n t}}}{\kappa^{2}+1} F(x)+\frac{\kappa e^{n t}-\kappa \sqrt{\kappa^{2}+1-\kappa^{2} e^{2 n t}}}{\kappa^{2}+1} N(x),
$$

que está definida para todo $t \in\left(-\infty, \frac{1}{2 n} \ln \left(\frac{\kappa^{2}+1}{\kappa^{2}}\right)\right)$. Além disso, a solução colapsa em um ponto quando $t \rightarrow \frac{1}{2 n} \ln \left(\frac{\kappa^{2}+1}{\kappa^{2}}\right)$.

Prova. Pelo Teorema 3.2 a solução é dada por

$$
\widehat{F}^{t}(x)=\cos (\xi(t)) F(x)+\sin (\xi(t)) N(x),
$$

onde $\xi(t)$ satisfaz (3.2), isto é,

$$
\xi^{\prime}(t)=n \frac{\sin (\xi(t))+\kappa \cos (\xi(t))}{\cos (\xi(t))-\kappa \sin (\xi(t))}
$$

assim

$$
\frac{d}{d t} \ln |\sin (\xi(t))+\kappa \cos (\xi(t))|=n .
$$

Integrando a equação, como $\xi(0)=0$, temos

$$
\sin (\xi(t))+\kappa \cos (\xi(t))=\kappa e^{n t} .
$$

Isolando $\sin (\xi(t))$ e elevando ao quadrado, temos

$$
\sin ^{2}(\xi(t))=\kappa^{2}\left(e^{n t}-\cos (\xi(t))\right)^{2},
$$


que em termos de $\cos (\xi(t))$, reduz-se a

$$
\left(\kappa^{2}+1\right) \cos ^{2}(\xi(t))-2 \kappa^{2} e^{n t} \cos (\xi(t))+\kappa^{2} e^{2 n t}-1=0 .
$$

Portanto, segue (3.6) e a solução esta definida para todo $t<\frac{1}{2 n} \ln \left(\frac{\kappa^{2}+1}{\kappa^{2}}\right)$.

Agora, vamos provar que esta solução colapsa em um ponto quando $t \rightarrow \frac{1}{2 n} \ln \left(\frac{\kappa^{2}+1}{\kappa^{2}}\right)$. Considere $\kappa>0$ e seja $\left\{E_{1}, \ldots, E_{n}\right\}$ uma base ortonormal da segunda forma fundamental $h$ de $F(M)$. Pelo Lema 1.15, temos

$$
\begin{aligned}
\widehat{g}^{t}\left(E_{\imath}, E_{\jmath}\right) & =(\cos (\xi(t))-\kappa \sin (\xi(t)))^{2} \delta_{\imath \jmath} \\
& =\left(\kappa^{2}+1-e^{2 n t}\right)^{2} \delta_{\imath \jmath} .
\end{aligned}
$$

Assim, fazendo $t^{*}=\frac{1}{2 n} \ln \left(\frac{\kappa^{2}+1}{\kappa^{2}}\right)$, temos

$$
\lim _{t \rightarrow t^{*}} \widehat{g}^{t}\left(E_{\imath}, E_{\jmath}\right)=0
$$

para todo $\imath, \jmath=1, \ldots, n$. Portanto, quando $t \rightarrow t^{*}, \widehat{F}^{t}(M)$ converge para um ponto de $\mathbb{S}^{n+1}$.

Proposição 3.15 Seja $F: \mathbb{S}^{1} \times \mathbb{S}^{1} \rightarrow \mathbb{S}^{3} \subset R^{4}$ a imersão de um Toro de Hopf na esfera $\mathbb{S}^{3}$, com campo normal unitário $N$ e curvaturas principais $\kappa_{1}$ e $\kappa_{2}$, satisfazendo $\kappa_{1} \kappa_{2}=-1$. Então a solução do fluxo de curvatura média por hipersuperfícies paralelas sobre $F\left(\mathbb{S}^{1} \times \mathbb{S}^{1}\right)$ é

$$
\widehat{F}^{t}(x)=\cos (\xi(t)) F(x)+\sin (\xi(t)) N(x),
$$

onde

$$
\begin{aligned}
\cos (\xi(t)) & =\sqrt{\frac{H^{2}+4+H^{2} e^{4 t}+2 \sqrt{H^{2}+4-H^{2} e^{8 t}}}{2\left(H^{2}+4\right)}} \\
e & \sin (\xi(t))=\operatorname{sgn}(H) \sqrt{\frac{H^{2}+4-H^{2} e^{4 t}-2 \sqrt{H^{2}+4-H^{2} e^{8 t}}}{2\left(H^{2}+4\right)}}
\end{aligned}
$$

que está definida para todo $t \in\left(-\infty, \frac{1}{8} \ln \left(\frac{H^{2}+4}{H^{2}}\right)\right)$, onde $H=\kappa_{1}+\kappa_{2}$. Além disso, a solução colapsa em uma geodésica de $\mathbb{S}^{3}$, quando $t \rightarrow \frac{1}{8} \ln \left(\frac{H^{2}+4}{H^{2}}\right)$. 
Prova. Pelo Teorema 3.2, a solução do fluxo de curvatura média sobre $F(M)$ é dado por

$$
\widehat{F}^{t}(x)=\cos (\xi(t)) F(x)+\sin (\xi(t)) N(x),
$$

onde $\xi(t)$ satisfaz (3.2), portanto

$$
\begin{aligned}
\xi^{\prime}(t) & =\frac{\sin (\xi(t))+\kappa_{1} \cos (\xi(t))}{\cos (\xi(t))-\kappa_{1} \sin (\xi(t))}+\frac{\sin (\xi(t))+\kappa_{2} \cos (\xi(t))}{\cos (\xi(t))-\kappa_{2} \sin (\xi(t))} \\
& =\frac{4 \cos (\xi(t)) \sin (\xi(t))+H\left(\cos ^{2}(\xi(t))-\sin ^{2}(\xi(t))\right)}{\cos ^{2}(\xi(t))-\sin ^{2}(\xi(t))-H \cos (\xi(t)) \sin (\xi(t))} \\
& =2 \frac{2 \sin (2 \xi(t))+H \cos (2 \xi(t))}{2 \cos (2 \xi(t))-H \sin (2 \xi(t))}
\end{aligned}
$$

Assim

$$
\frac{d}{d t} \ln |2 \sin (2 \xi(t))+H \cos (2 \xi(t))|=4
$$

Integrando esta equação, $\operatorname{com} \xi(0)=0$, temos

$$
2 \sin (2 \xi(t))+H \cos (2 \xi(t))=H e^{4 t} .
$$

Elevando ao quadrado e reescrevendo a equação em termos de $\cos (2 \xi(t))$, temos

$$
\left(H^{2}+4\right) \cos ^{2}(2 \xi(t))-2 H^{2} e^{4 t} \cos (2 \xi(t))+H^{2} e^{8 t}-4=0 .
$$

Resolvendo esta equação em termos de $\cos (2 \xi(t))$, como $\xi(0)=0$, temos

$$
\cos (2 \xi(t))=\frac{H^{2} e^{4 t}+2 \sqrt{H^{2}+4-H^{2} e^{8 t}}}{H^{2}+4}
$$

e substituindo em (3.8), obtemos

$$
\sin (2 \xi(t))=\frac{-2 H e^{4 t}+H \sqrt{H^{2}+4-H^{2} e^{8 t}}}{H^{2}+4} .
$$

Portanto, obtemos (3.7) e a solução está definida para todo $t \leq \frac{1}{8} \ln \left(\frac{H^{2}+4}{H^{2}}\right)$.

Agora, vamos provar que a solução colapsa em uma geodésica quando $t \rightarrow t^{*}=$ $\frac{1}{8} \ln \left(\frac{H^{2}+4}{H^{2}}\right)$. Para isso, considere uma base $\left\{E_{1}, E_{2}\right\}$ de autovetores de $h$ de tal modo que $E_{\imath}$ é o autovetor associado ao autovalor $\kappa_{\imath}$, para $\imath=1,2$. Além disso, sem perda de generalidade, podemos considerar $\kappa_{1}<0<\kappa_{2}$. Desta forma, temos que

$$
\lim _{t \rightarrow t^{*}} \cos (\xi(t))=\sqrt{\frac{\kappa_{2}}{\kappa_{2}-\kappa_{1}}} \text { e } \lim _{t \rightarrow t^{*}} \sin (\xi(t))=\operatorname{sng}(H) \sqrt{\frac{-\kappa_{1}}{\kappa_{2}-\kappa_{1}}}
$$


. Assim, pelo Lema 1.15, temos que

$$
\lim _{t \rightarrow t^{*}} \widehat{g}^{t}\left(E_{1}, E_{1}\right)=-\kappa_{1}\left(\kappa_{2}-\kappa_{1}\right)
$$

e

$$
\lim _{t \rightarrow t^{*}} \widehat{g}^{t}\left(E_{2}, E_{2}\right)=0
$$

desta forma a solução colapsa em uma curva. Além disso,

$$
\lim _{t \rightarrow t^{*}} \widehat{h}^{t}\left(E_{1}, E_{1}\right)=\left(\sqrt{\frac{-\kappa_{1}}{\kappa_{2}-\kappa_{1}}}+\kappa_{1} \sqrt{\frac{\kappa_{2}}{\kappa_{2}-\kappa_{1}}}\right)^{2}=0 .
$$

Portando, a solução colapsa em uma geodésica da esfera $\mathbb{S}^{2}$.

Exemplo 3.16 Considere o toro de Hopf em $\mathbb{S}^{3}$ parametrizado por

$$
F(\theta, \phi)=\left(\frac{\sqrt{6}}{3} \cos \theta, \frac{\sqrt{6}}{3} \sin \theta, \frac{\sqrt{3}}{3} \cos \phi, \frac{\sqrt{3}}{3} \sin \phi\right) .
$$

O campo normal unitário a $F$ é

$$
N(\theta, \phi)=\left(-\frac{\sqrt{3}}{3} \cos \theta,-\frac{\sqrt{3}}{3} \sin \theta, \frac{\sqrt{6}}{3} \cos \phi, \frac{\sqrt{6}}{3} \sin \phi\right),
$$

e as curvaturas principais com respeito a $N$ são $\kappa_{1}=-\frac{\sqrt{2}}{2}$ e $\kappa_{2}=\sqrt{2}$. Assim, a solução do fluxo de curvatura média sobre $F$ é dada por

$$
\widehat{F}^{t}(x)=\frac{\sqrt{2}}{6} \sqrt{9+e^{4 t}+2 \sqrt{18-2 e^{8 t}}} F(x)+\frac{\sqrt{2}}{6} \sqrt{9-e^{4 t}-2 \sqrt{18-2 e^{8 t}}} N(x),
$$

que pode ser visualizado, via projeção estereográfica, na Figura 3.7.

Observação 3.17 Para obter a solução do fluxo de curvatura média sobre as outras hipersuperfícies isoparamétricas da esfera, basta integrar (3.2) e substituir $\xi(t)$ em

$$
\widehat{F}^{t}(x)=\cos (\xi(t)) F(x)+\sin (\xi(t)) N(x) .
$$



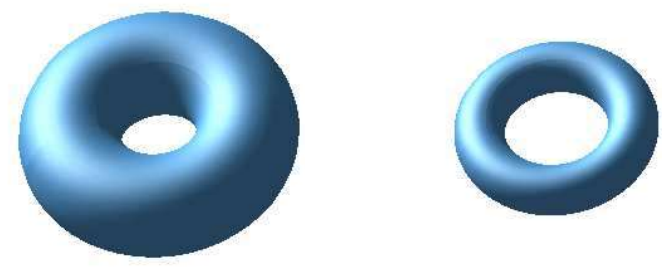

$\bullet \bullet \bullet$

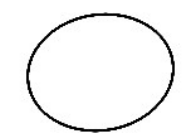

Figura 3.7: Fluxo de Curvatura Média sobre o Toro de Hopf 


\section{Referências}

[1] Abresch, U.; Langer, J. The normalized curve shortening flow and homothetic solutions. J. Diff. Geom. 23 (1986), no. 2, 175-196. MR 845704

[2] Angenent, S. B., Curve shortening and the topology of closed geodesics on surfaces. Annals of Math. 162 (2005), 1187-1241. MR 2179729

[3] Angenent, S. B., Shrinking doughnuts., Progress Nonlinear Diff. Eq. Appl., vol. 7, 21-38. Birkhäuser Boston, Boston (1992). MR 1167827

[4] Altschuler, S. J., Singularities of the curve shrinking flow for space curves. J Diff. Geom. 34 (1991), no. 2, 491-514. MR 1131441

[5] Bryan, P.; Louie, J., Classification of convex ancient solutions to curve shortening flow on the sphere. J. Geom. Anal. 26 (2016), no. 2, 858-872. MR 3472819

[6] Cartan, E., Familles de surfaces isoparamétriques dans les espaces à courbure constante. Ann. Mat. Pura Appl. (4) 17 (1938), 177-191.

[7] Chopp, D.L., Computation of self-similar solutions for mean curvature flow. Exp. Math. 3(1) (1994), 1-15. MR 1302814

[8] Clutterbuck, J., Schnürer, O.C., Schulze, F., Stability of translating solutions to mean curvature flow. Calc. Var. Partial Differ. Equ. 29 (2007), 281-293 . MR 2321890 
[9] Colding, T. H.; Minicozzi II, W. P.; Pedersen, E. K., Mean curvature flow. Bull. Amer. Math. Soc. (N.S.) 52 (2015), no. 2, 297-333. MR 3312634

[10] Colding, T. H. ; Minicozzi, W. P.; Pedersen, E.K., Mean curvature flow as a tool to study topology of 4-manifolds. preprint. Available at arXiv:1208.5988.

[11] Ecker, K.; Huisken, G., Parabolic methods for the construction of spacelike slices of prescribed mean curvature in cosmological spacetimes. Comm. Math. Phys. 135 (1991), no. 3, 595-613. MR 1091580

[12] Gage, M. E., Curve shortening makes convex curves circular, Invent. Math. 76 (1984), 357-364. MR 084401

[13] Gage, M., Curve shortening on surfaces. Ann. Sci. École Norm. Sup. (4) 23 (1990), no. 2, 229-256. MR1046497

[14] Gage, M.; Hamilton, R., The heat equation shrinking convex plane curves. J. Diff. Geom., 23 (1986), 69-96.

[15] Giga Y., Surface evolution equations. A level set approach, Monographs in Mathematics, vol. 99, Birkhauser, Basel, 2006.

[16] Halldorsson, H. P., Self-similar solutions to the curve shortening flow. Trans. Amer. Math. Soc. 364 (2012), no. 10, 5285-5309. MR 2931330

[17] Halldorsson, H. P., Helicoidal surfaces rotating/translating under the mean curvature flow. Geom. Dedicata 162 (2013), 45-65. MR 3009534

[18] Halldorsson, H. P., Self-similar solutions to the curve shortening flow in the Minkowski plane $\mathbb{R}^{1,1}$. J. Reine Angew. Math. 704 (2015), 209-243. MR 3365779

[19] Huisken, G.; Yau, S. T., Definition of center of mass for isolated physical systemsand unique foliations by stable spheres with constant mean curvature. Invent. Math. 124 (1996), 281-311.

[20] Huisken, G., Asymptotic behavior for singularities of the mean curvature flow, J. Differential Geom. 31 (1990), no. 1, 285-299. 
[21] Hungerbühler, N.; Smoczyk, K., Soliton solutions for the mean curvature flow. Diff. Int. Eq. 13 (2000), no. 10-12, 1321-1345. MR 1787070

[22] Ilmanen, T.; White,B., Sharp lower bounds on density for area-minimizing cones. preprint. Available at arXiv:10105068.

[23] Ilmanen,T., Singularities of mean curvature flow of surfaces, 1995. Available at http://www.math.ethz.ch/ ilmanen/papers/pub.html

[24] Ishimura, N., Curvature evolution of plane curves with prescribed opening angle. Bull. Austral. Math. Soc., 52 (1995) no. 2, 287-296. MR 1348488

[25] Kleene, S.; Møller, N. M., Self-shrinkers with a rotational symmetry. Trans. Amer. Math. Soc. 366 (2014), no. 8, 3943-3963. MR 3206448

[26] Lima, E., Curso de Análise. 1 (2004) Projeto Euclides, IMPA.

[27] Liu, X.; Terng, C. -L., The mean curvature flow for isoparametric submanifolds, Duke Math. J., 147 (2009), no. 1, 157-179.

[28] Mantegazza, C., Lecture notes on mean curvature flow. Progress im Mathematics, vol. 290, Birkhauser/Springer Basel AG, Basel, 2011. MR 2815949

[29] Mullins, W. M., Two-dimensional motion of idealized grain boundaries. J. Appl. Phys. 27 (1956), 900-904. MR 0078836

[30] Nguyen, X. H., Translating tridents. Commun. Partial Differ. Equ. 34 (2009), 257280. MR 2512861

[31] Segre B., Famiglie di ipersuperficie isoparametrische negli spazi euclidei ad un qualunque numero di demesioni. Atti. Accad. naz Lincie Rend. Cl. Sci. Fis. Mat. Natur. 27 (1938), 203-207.

[32] Slotine, J. R.; Li, W., Applied nonlinear control, (1991) Prentice Hall, New Jersey.

[33] Somigliana C., Sulle relazione fra il principio di Huygens e l'ottica geometrica, Atti Acc. Sc. Torino 54 (1918-1919), 974-979 (see also in Memorie Scelte, 434-439). 
[34] Sotomayor, J., Lições de equações diferenciais ordinárias, Inst. Mat. Pura e Apl. (Projeto Euclides), 2 (1979).

[35] Urbas, J., Complete noncompact self-similar solutions of Gauss curvature flow. I. Positive powers. Math. Ann. 311 (1998), no. 2, 251-274. MR 1625754

[36] Wang, X.-J. Convex solutions to the mean curvature flow. Ann. Math. (2) 173 (2011), no 3, 1185-1239. MR 2800714 